Supporting information

\title{
Lithium-Promoted Cycloaddition of Indole-2,3-dienolates and Carbon Disulfide as a One-Pot Route to Thiopyrano[4,3- $b]$ indole-3(5H)- thiones
}

Konstantin F. Suzdaleva ${ }^{\mathrm{a}}$, Julia V. Vyalyh ${ }^{\mathrm{a} *}$, Valery V. Tkachever, Ekaterina A. Lysenko ${ }^{\mathrm{a}}$, Oleg N. Burov ${ }^{\mathrm{a}}$, Anton V. Lisovin ${ }^{\mathrm{a}}$, Mikhail E. Kletskii ${ }^{\mathrm{a}}$, and Sergey V. Kurbatov ${ }^{\mathrm{a}}$

${ }^{a}$ Department of Chemistry, Southern Federal University, Rostov-on-Don 344090, Russia

bInstitute of Problems of Chemical Physics, Russian Academy of Sciences, Chernogolovka 142432, Russia 
Table of contents

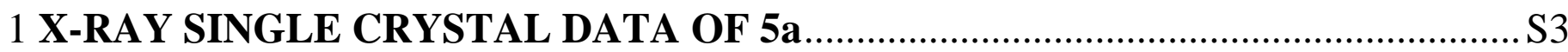

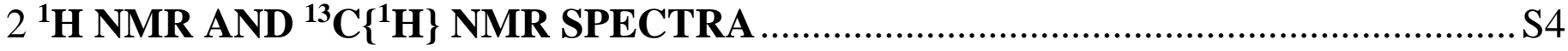

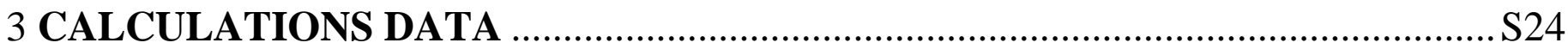




\section{X-RAY SINGLE CRYSTAL DATA OF 5a}

Single crystal of compound 5a was obtained by slow evaporation of the acetonitrile solution. Monoclinic crystals; chemical formula moiety: $\mathrm{C}_{18} \mathrm{H}_{13} \mathrm{NS}_{2}, \mathrm{M}=307.41$; a 6.11819(17), b 17.4389(5), c 13.6709(5) Á, ß 94.169(3) ${ }^{\circ}, \mathrm{V} 1454.74(8) \AA^{3}, \mathrm{Z} 4, \mathrm{~d}_{\text {calc }} 1.404 \mathrm{~g} / \mathrm{cm}^{3}, \mathrm{P} 21 / \mathrm{c}$ space group. Intensities of 20248 reflections were measured in the reciprocal space $\left(2 \theta \leq 67^{\circ}\right)$ using Iw-scanning. After exclusion of systematically cancelled reflections and averaging intensities of equivalent reflections, the working array of measured $\mathrm{F}^{2}(\mathrm{hkl})$ and $\sigma\left(\mathrm{F}^{2}\right)$ reflections contained 5930 independent reflections, 4489 of which with $\mathrm{F}^{2}>4 \sigma\left(\mathrm{F}^{2}\right)$. The structure was solved with the direct method and was refined by the full-matrix least-squares procedure with respect to $\mathrm{F}^{2}$ in anisotropic approximation for non-hydrogen atoms (hydrogen atoms isotropic) using SHELXTL program. ${ }^{*}$ The final refined parameters were $R_{1}=0.039$ for the reflections observed with $\mathrm{I} \geq 2 \sigma(\mathrm{i})$ and $\mathrm{R}_{1}=0.088$ for all measured reflections, $\mathrm{GOF}=$ 0.975 .

* Sheldrick, G. M. SHELXTL, v. 6.14 Structure Determination Software Suite; Bruker AXS: Madison, Wisconsin, USA, 2000. 
${ }^{1} \mathrm{H}$ NMR AND ${ }^{13} \mathrm{C}\left\{{ }^{1} \mathrm{H}\right\}$ NMR SPECTRA

${ }^{1} \mathrm{H} \mathrm{NMR}, \mathrm{CDCl}_{3}, 250 \mathrm{MHz}$

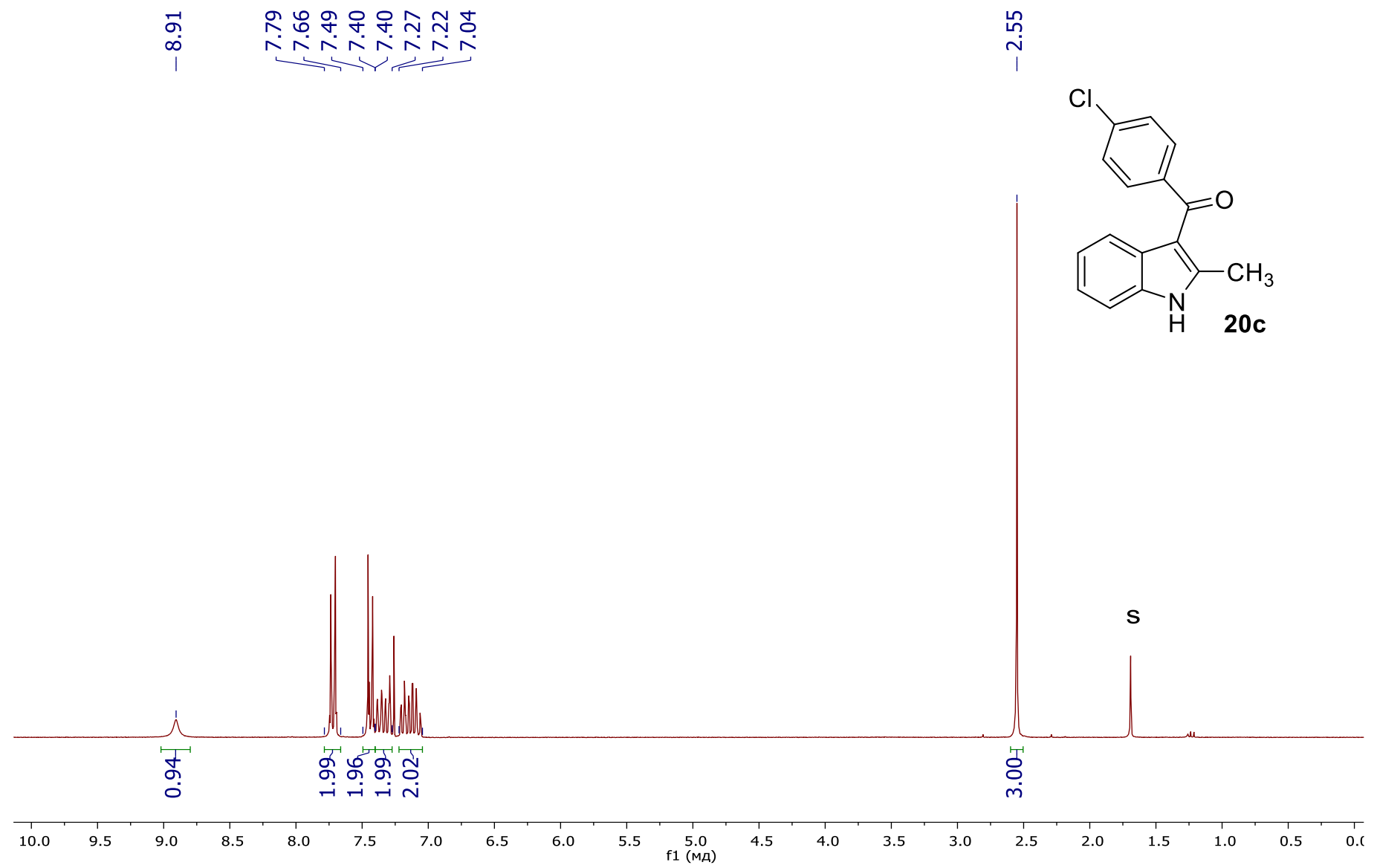

${ }^{13} \mathrm{C}\left\{{ }^{1} \mathrm{H}\right\} \mathrm{NMR}, \mathrm{CDCl}_{3}, 63 \mathrm{MHz}$

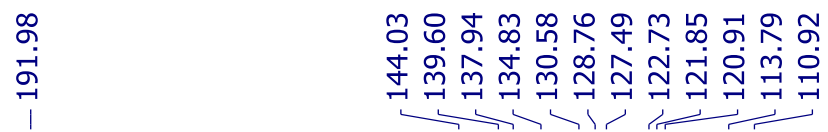

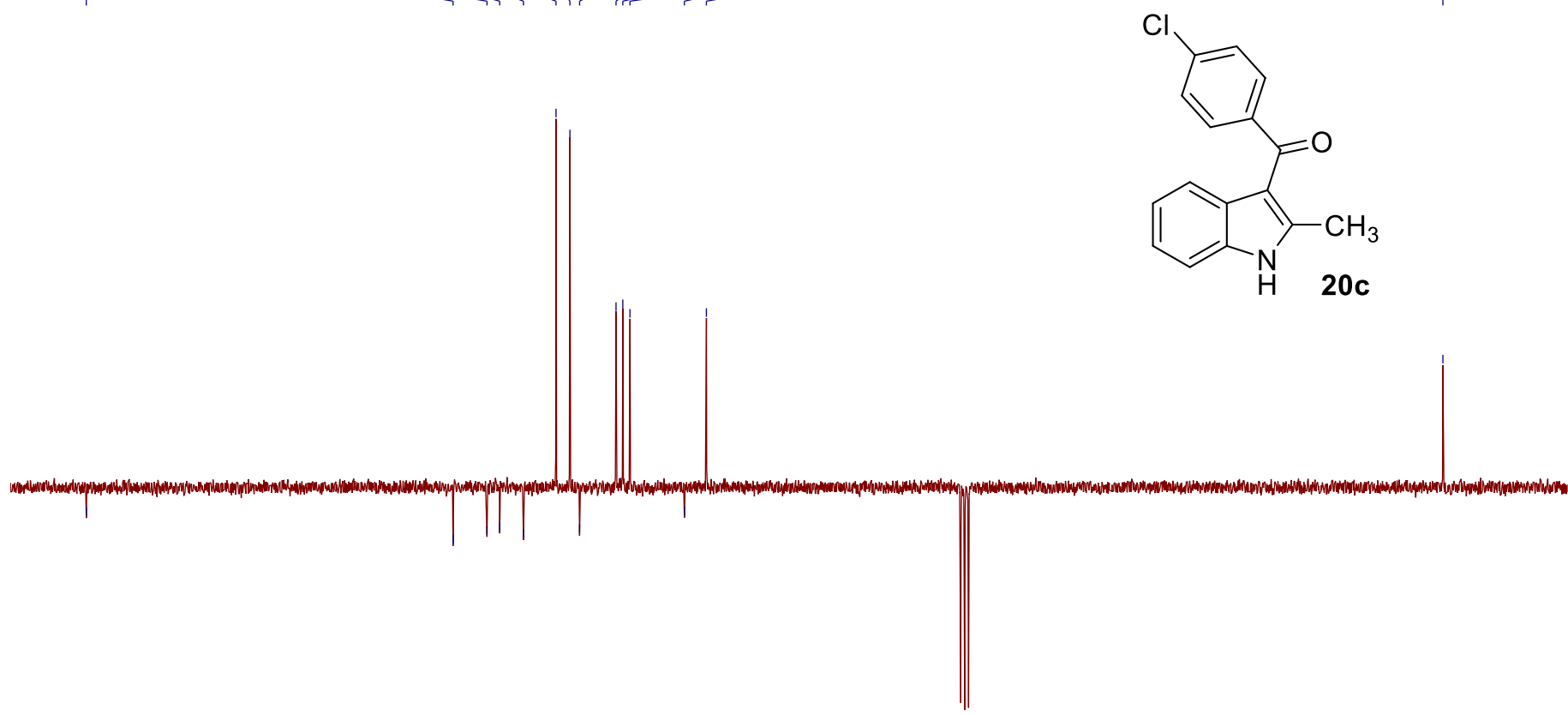

$\mathbf{S}$ 
${ }^{1} \mathrm{H} \mathrm{NMR}, \mathrm{CDCl}_{3}, 250 \mathrm{MHz}$

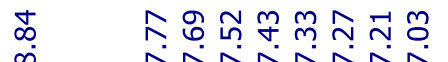

$\infty \quad$ N

$\begin{array}{ll}\text { Nָ} & \hat{m} \\ 1 & \text { I }\end{array}$<smiles>Cc1[nH]c2ccccc2c1C(=O)c1ccc(C(C)(C)C)cc1</smiles>
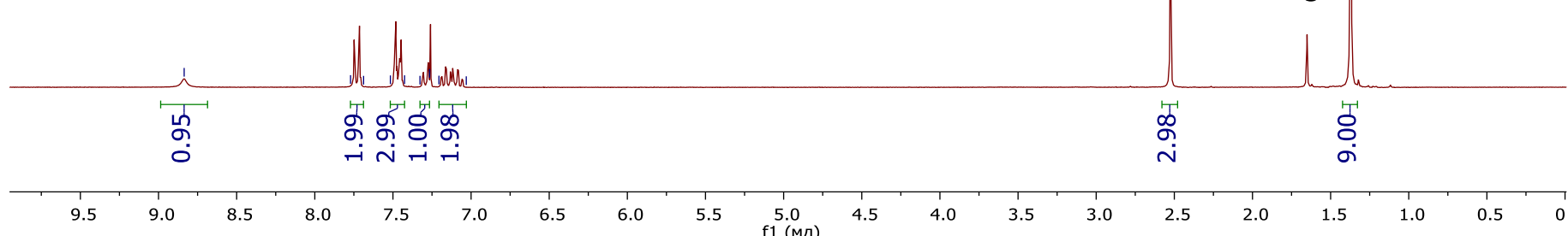

${ }^{13} \mathrm{C}\left\{{ }^{1} \mathrm{H}\right\} \mathrm{NMR}, \mathrm{CDCl}_{3}, 63 \mathrm{MHz}$
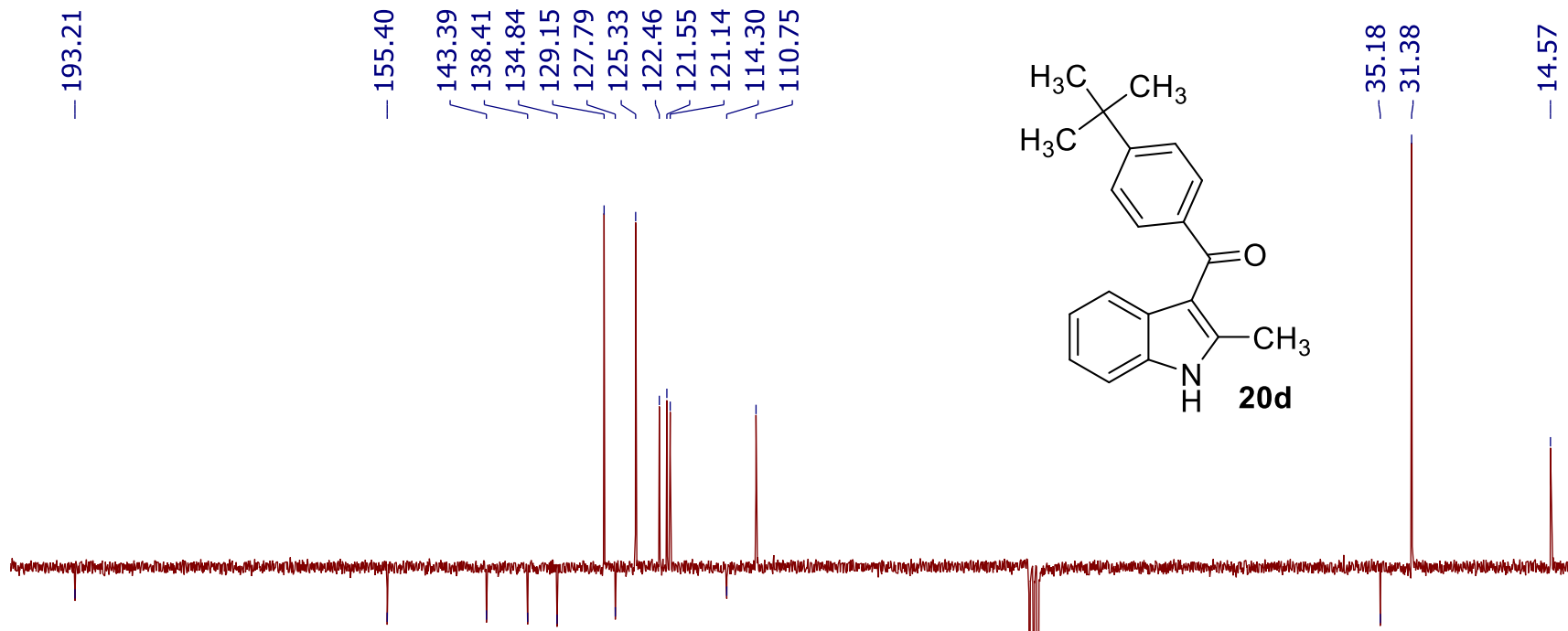

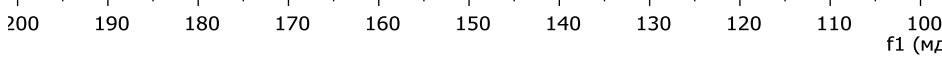


${ }^{1} \mathrm{H} \mathrm{NMR}, \mathrm{CDCl}_{3}, 600 \mathrm{MHz}$

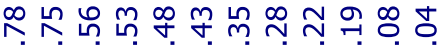

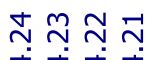

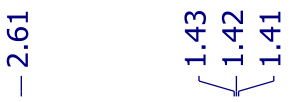

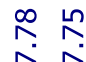

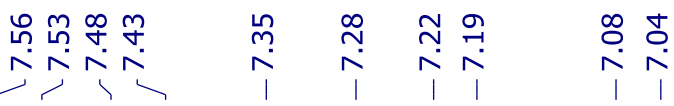
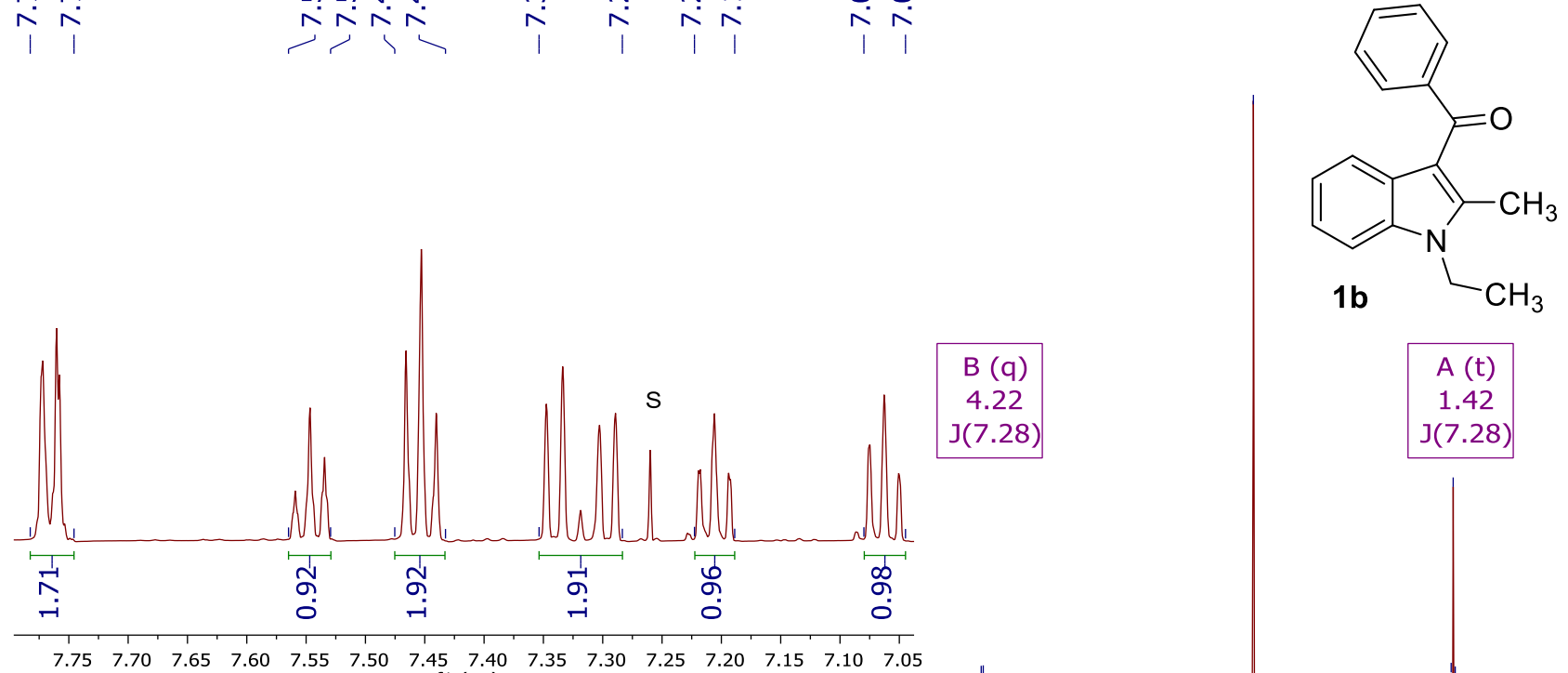

$\begin{array}{llllll} & \text { f1 (MA) }\end{array}$
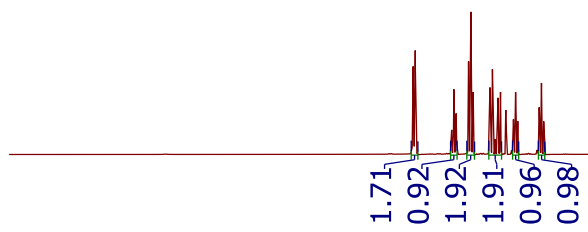

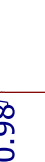

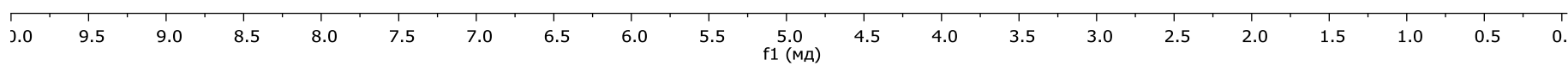

${ }^{13} \mathrm{C}\left\{{ }^{1} \mathrm{H}\right\} \mathrm{NMR}, \mathrm{CDCl}_{3}, 151 \mathrm{MHz}$

oิ

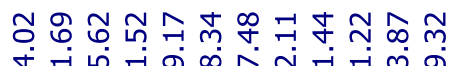

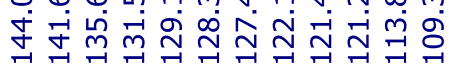
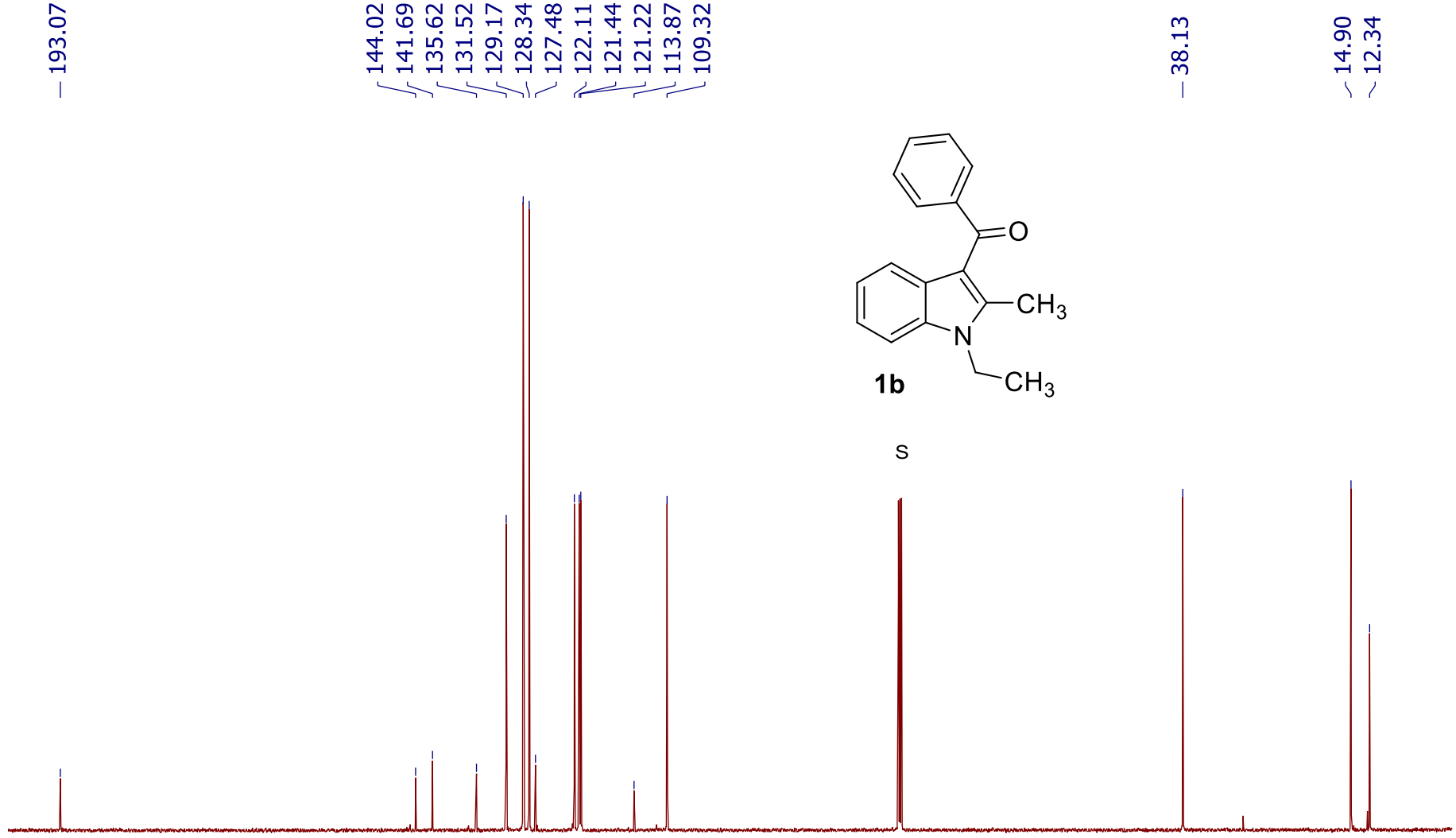
${ }^{1} \mathrm{H} \mathrm{NMR}, \mathrm{CDCl}_{3}, 250 \mathrm{MHz}$

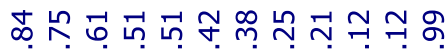

$\underset{\substack{\text { o } \\ \text { in } \\ \text { i }}}{c}$
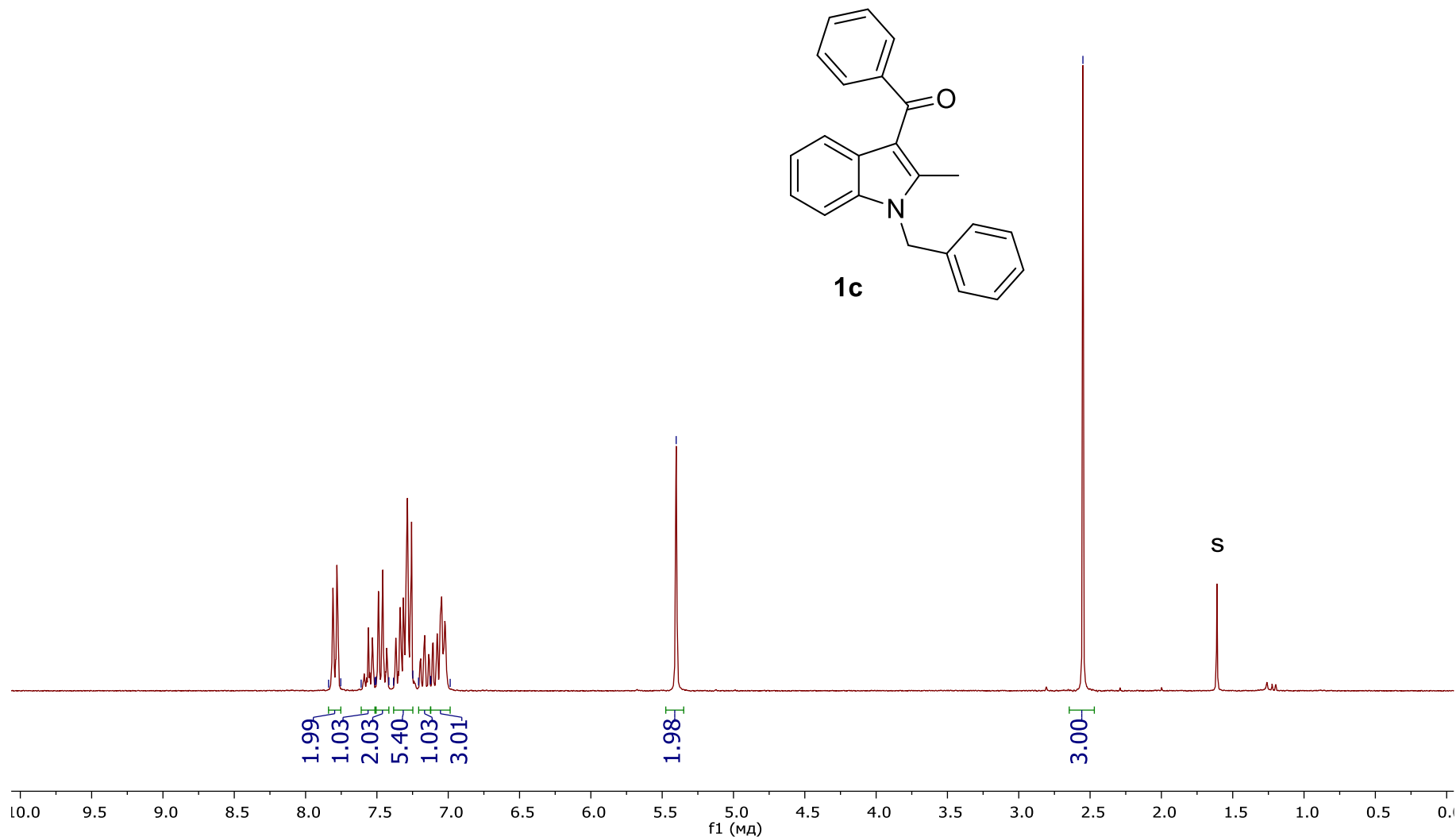

${ }^{13} \mathrm{C}\left\{{ }^{1} \mathrm{H}\right\} \mathrm{NMR}, \mathrm{CDCl}_{3}, 63 \mathrm{MHz}$
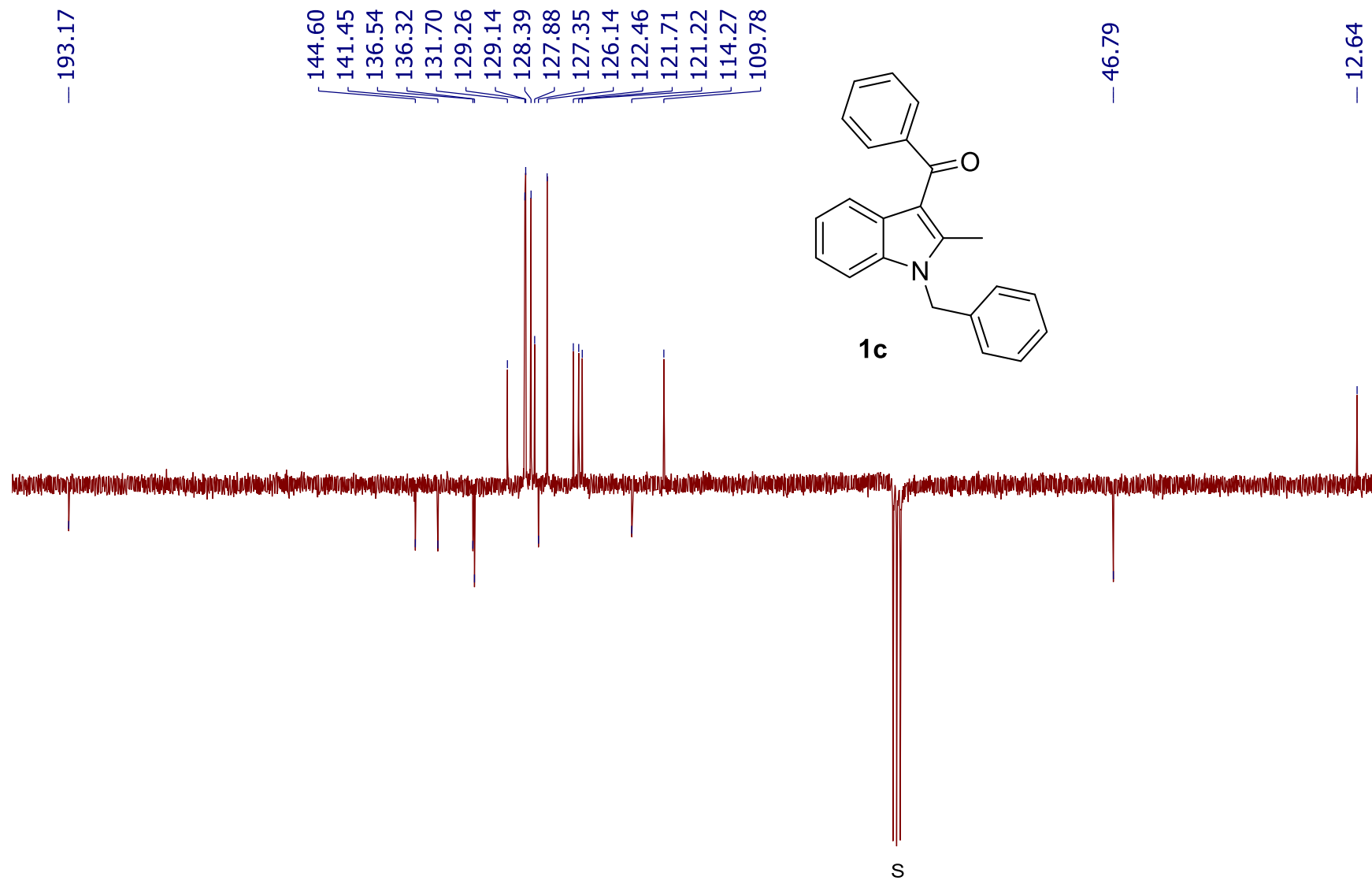

S

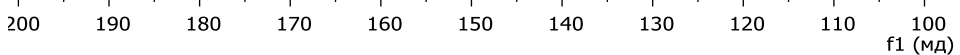


${ }^{1} \mathrm{H} \mathrm{NMR}, \mathrm{CDCl}_{3}, 300 \mathrm{MHz}$
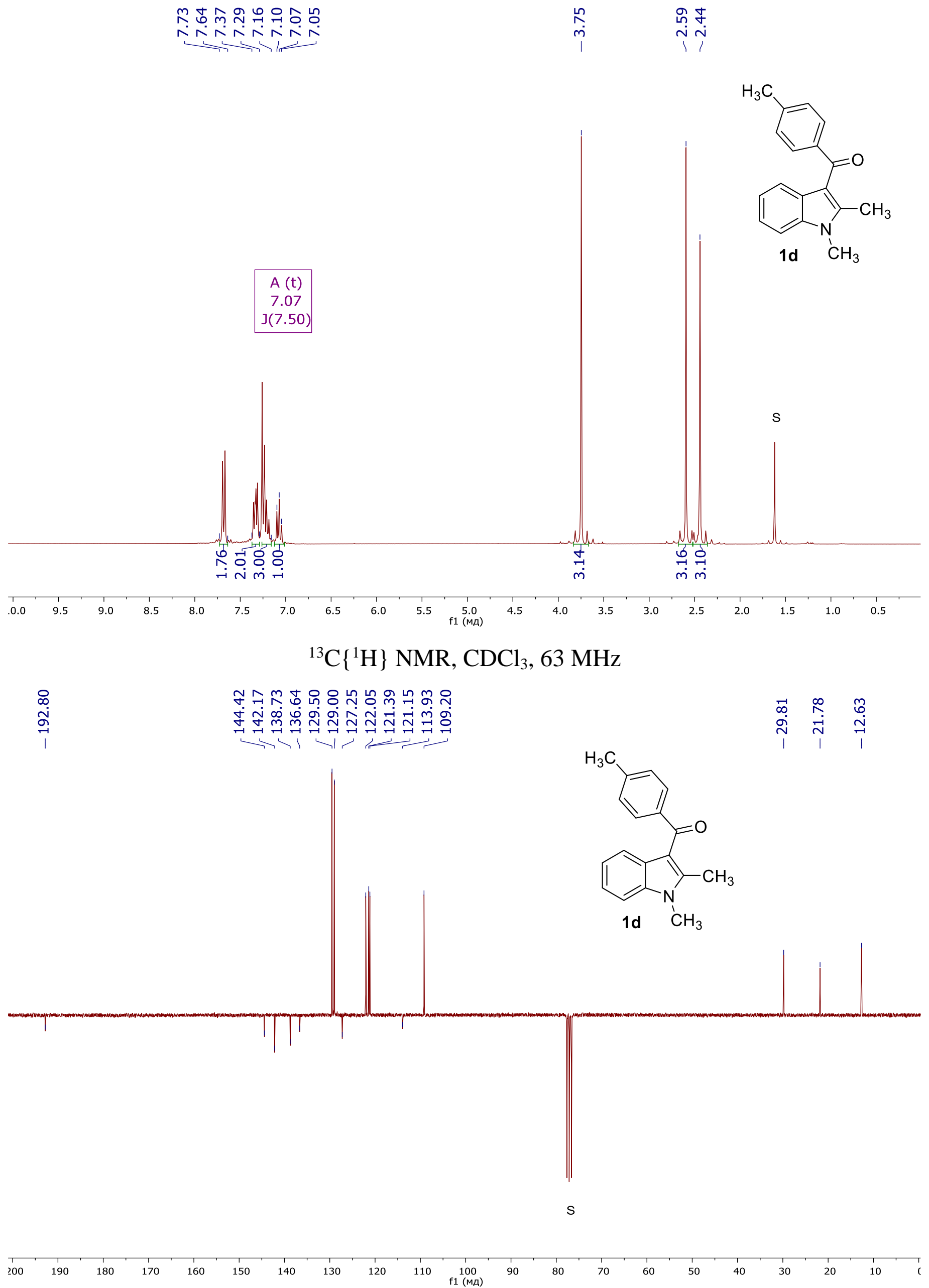
${ }^{1} \mathrm{H} \mathrm{NMR}, \mathrm{CDCl}_{3}, 250 \mathrm{MHz}$

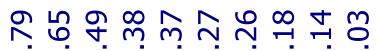

NNNNNNNN

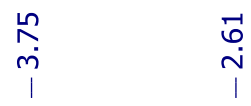

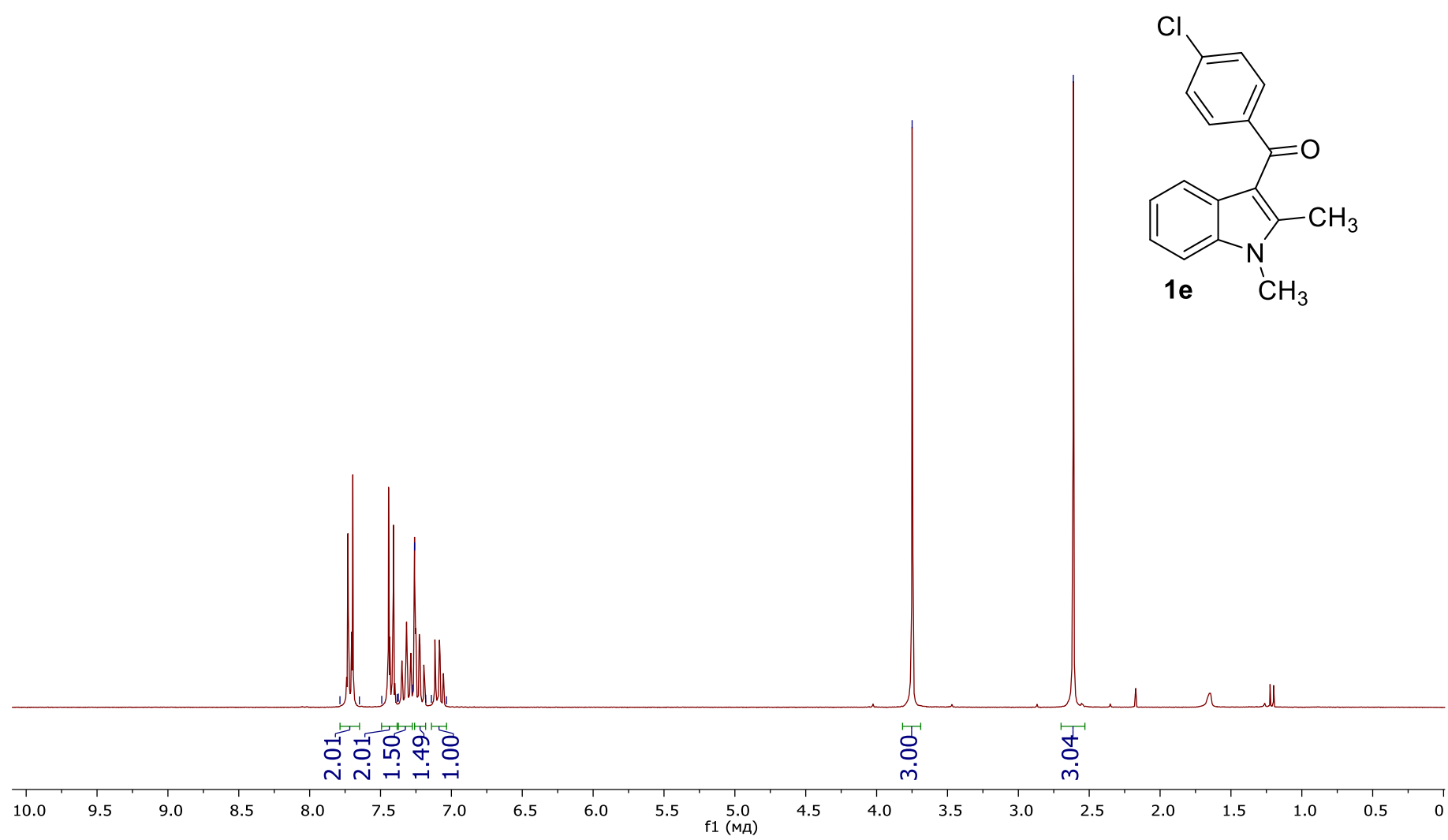

${ }^{13} \mathrm{C}\left\{{ }^{1} \mathrm{H}\right\} \mathrm{NMR}, \mathrm{CDCl}_{3}, 63 \mathrm{MHz}$

윰

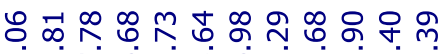

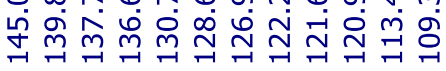

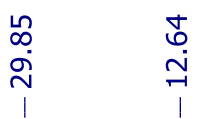

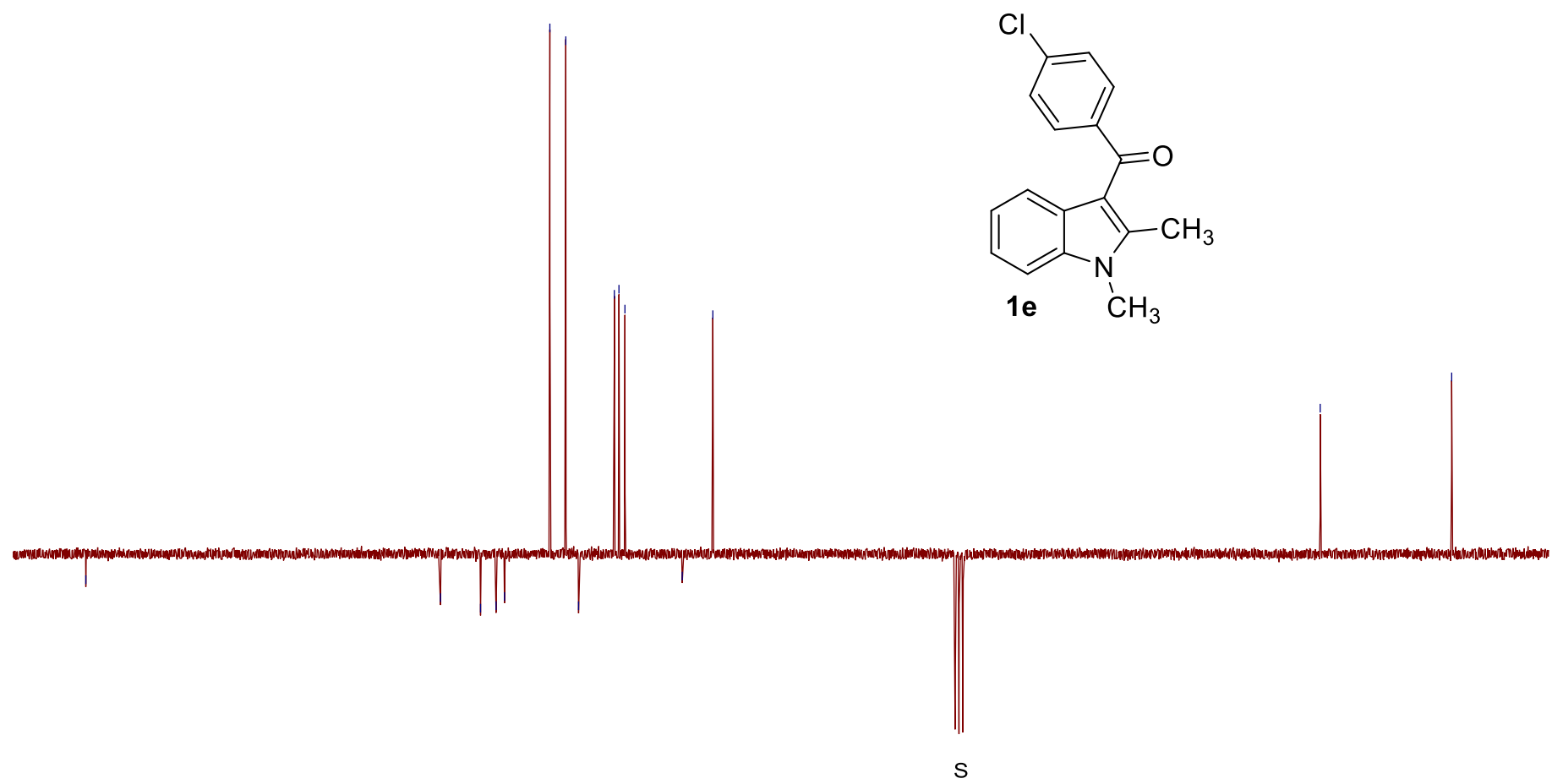

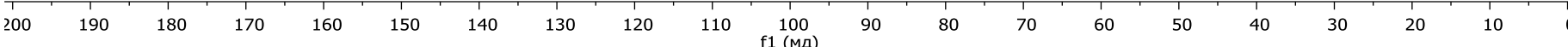


${ }^{1} \mathrm{H} \mathrm{NMR}, \mathrm{CDCl}_{3}, 250 \mathrm{MHz}$

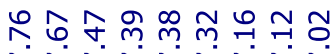

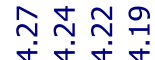

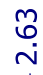

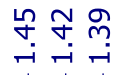

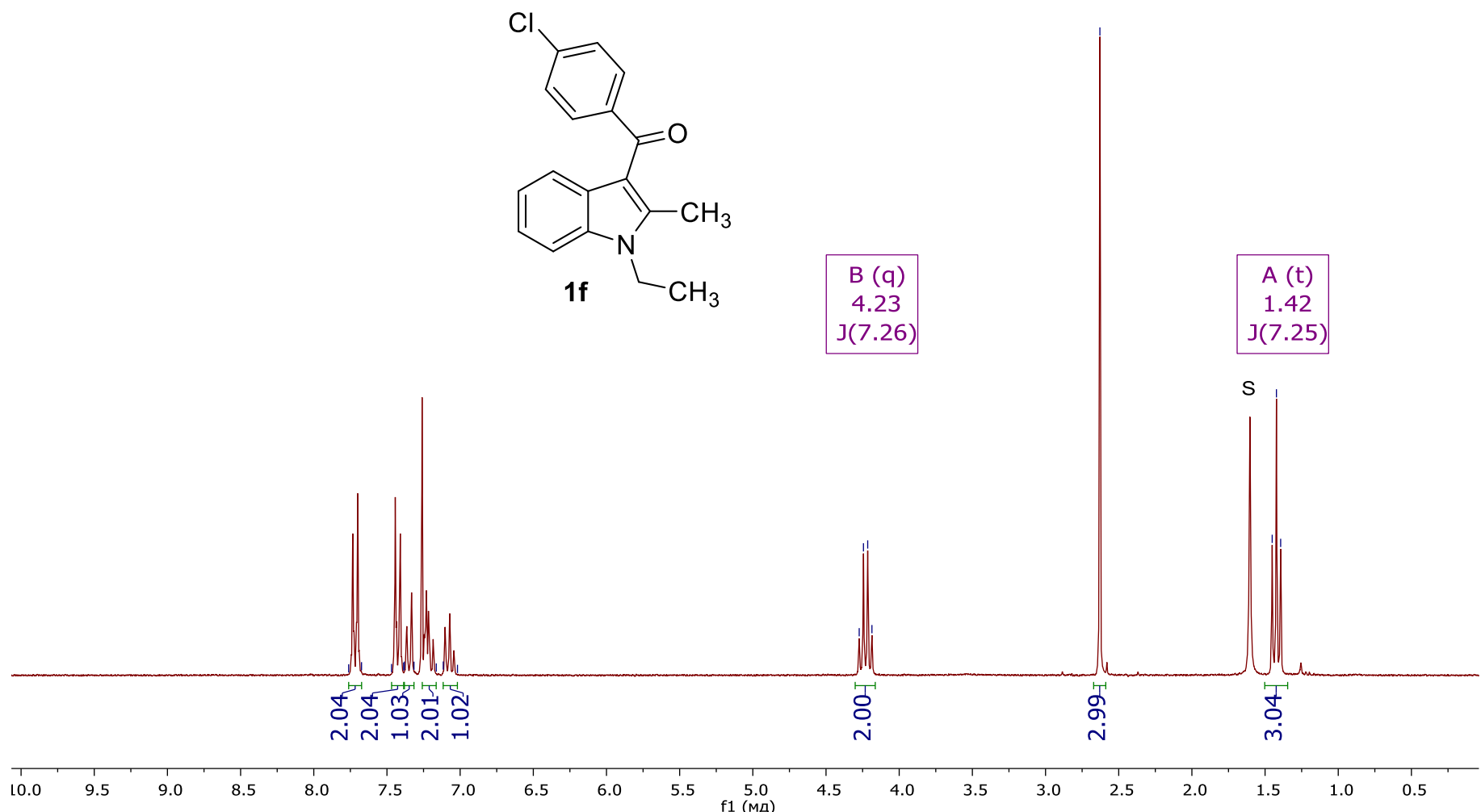

$\sqrt{2.24}$

${ }^{13} \mathrm{C}\left\{{ }^{1} \mathrm{H}\right\} \mathrm{NMR}, \mathrm{CDCl}_{3}, 63 \mathrm{MHz}$

$\overrightarrow{0}$
$\stackrel{-}{\sigma}$
$\stackrel{-}{1}$

ำ

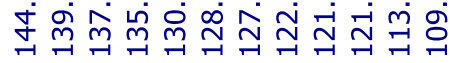

$\mathrm{Cl}$

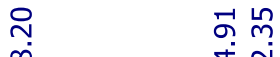

$7 \rightarrow-7,-7=7-7$<smiles>CCn1c(C)c(C(=O)c2ccc(Cl)cc2)c2ccccc21</smiles>

$\begin{array}{lllllllllllllllllllll}00 & 190 & 180 & 170 & 160 & 150 & 140 & 130 & 120 & 110 & 100 & 90 & 80 & 70 & 60 & 50 & 40 & 30 & 20 & 10 & 0\end{array}$ 
${ }^{1} \mathrm{H} \mathrm{NMR}, \mathrm{CDCl}_{3}, 250 \mathrm{MHz}$

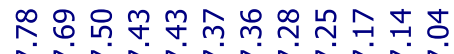

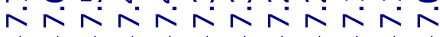

$\stackrel{+}{\stackrel{r}{r}}$

$\stackrel{\infty}{\stackrel{\infty}{\sim}}$

$\stackrel{\substack{m \\ i}}{i}$

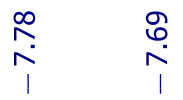

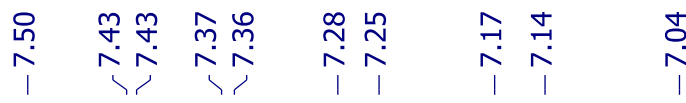
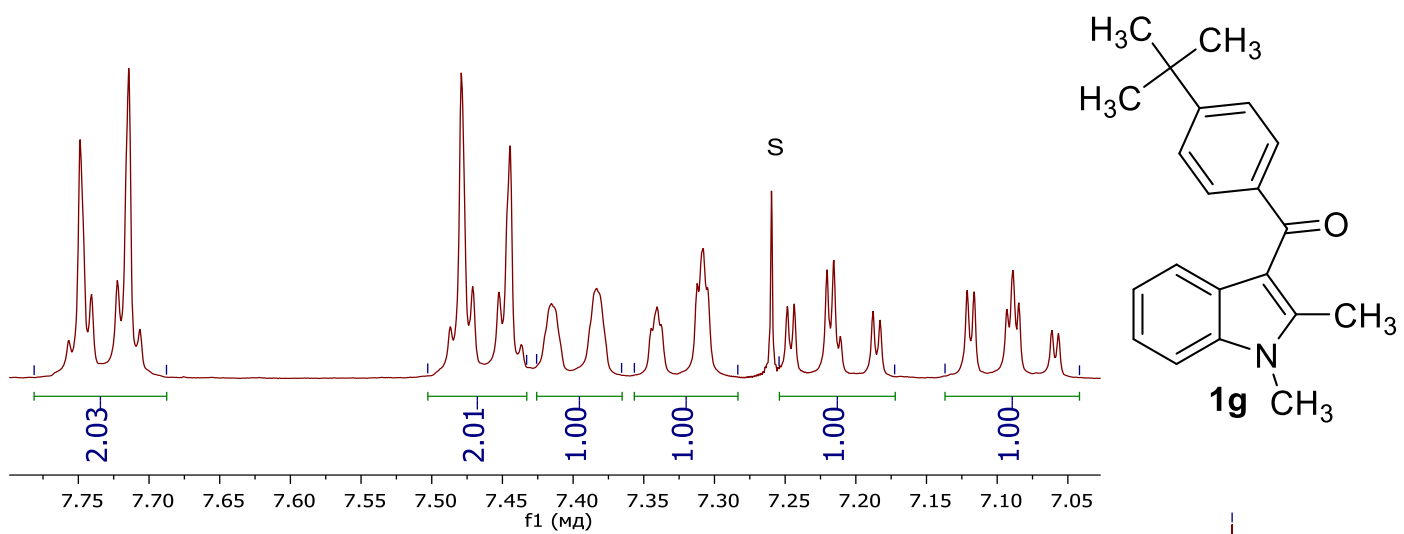

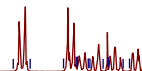

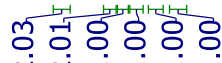
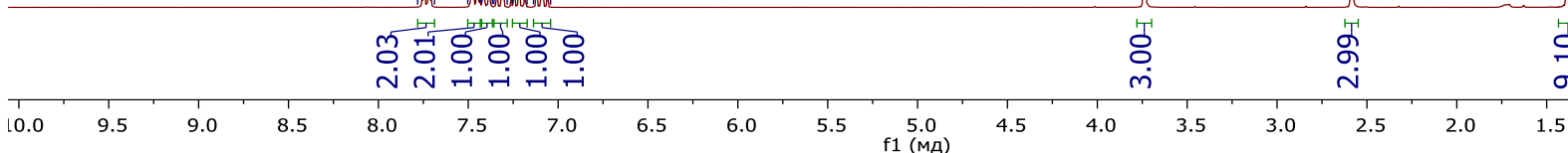

${ }^{13} \mathrm{C}\left\{{ }^{1} \mathrm{H}\right\} \mathrm{NMR}, \mathrm{CDCl}_{3}, 63 \mathrm{MHz}$

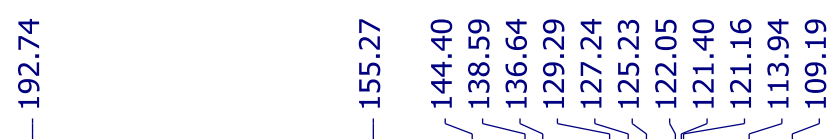

늑 $\infty$

मेंल

นำ
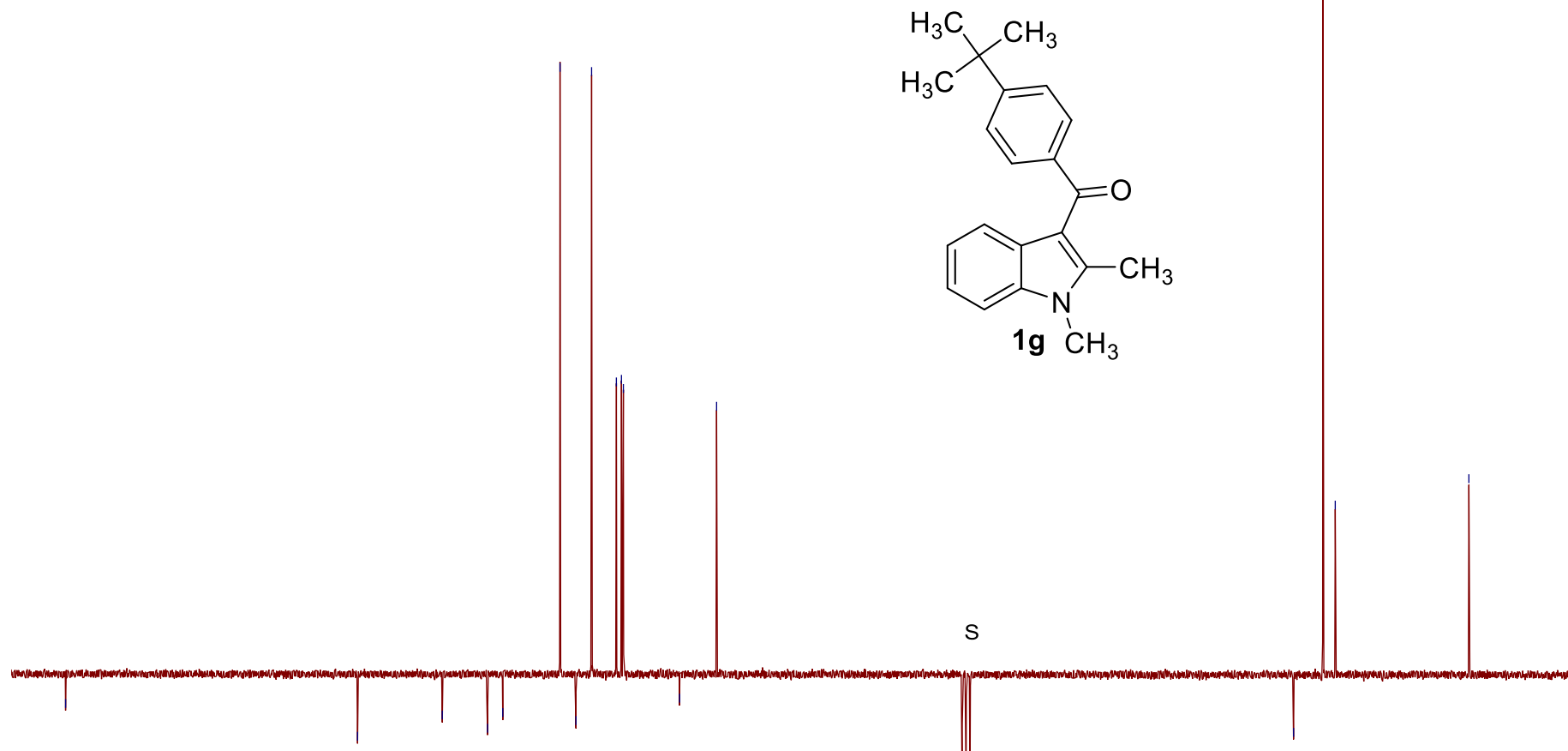

$\begin{array}{llllllllll}190 & 180 & 170 & 160 & 150 & 140 & 130 & 120 & 110 & 100\end{array}$ 
${ }^{1} \mathrm{H} \mathrm{NMR}, \mathrm{CDCl}_{3}, 250 \mathrm{MHz}$

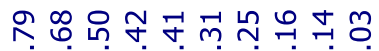

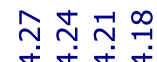

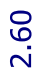

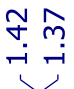

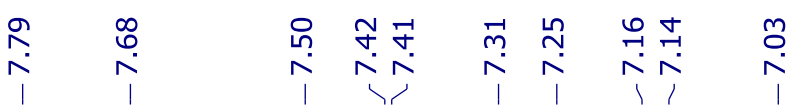
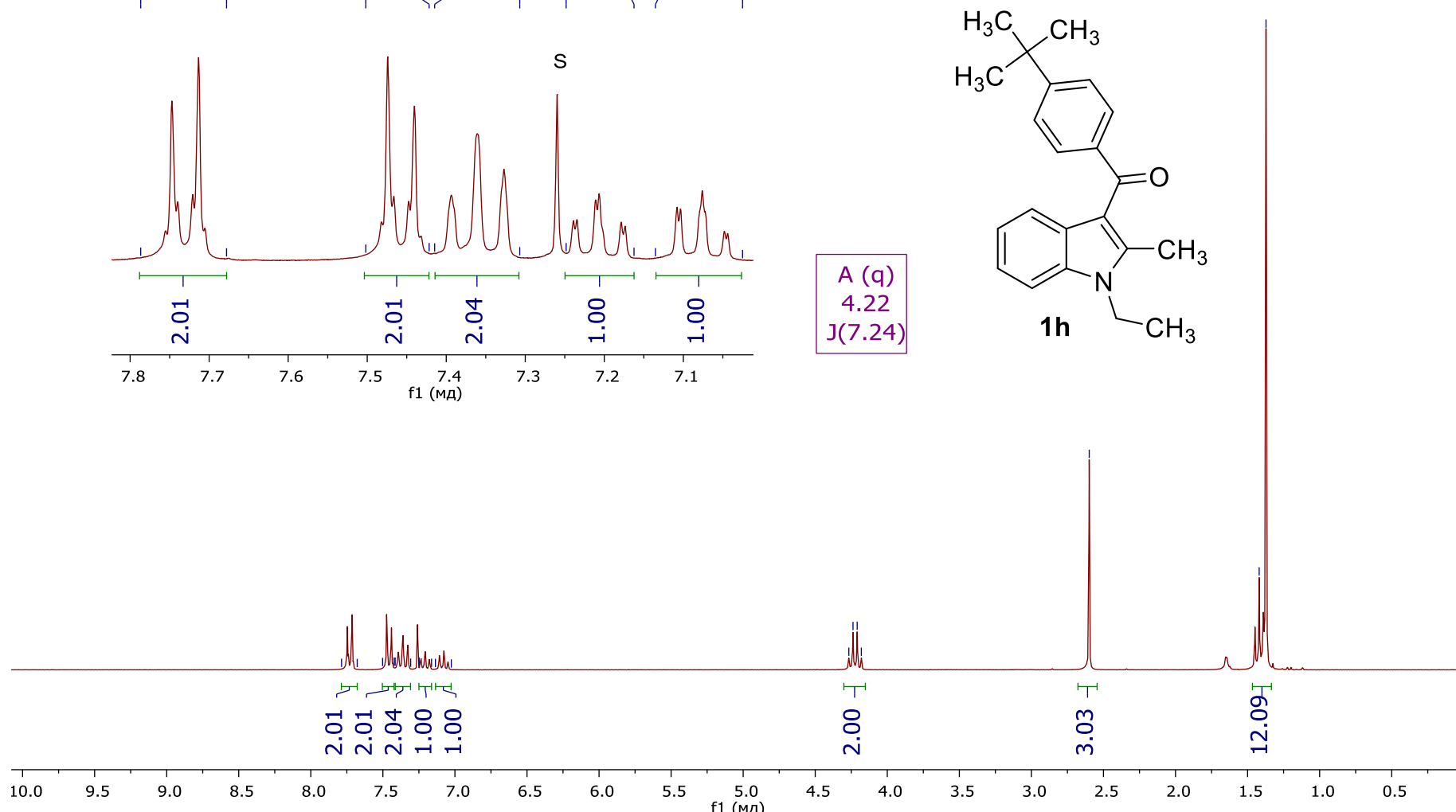

${ }^{13} \mathrm{C}\left\{{ }^{1} \mathrm{H}\right\} \mathrm{NMR}, \mathrm{CDCl}_{3}, 63 \mathrm{MHz}$

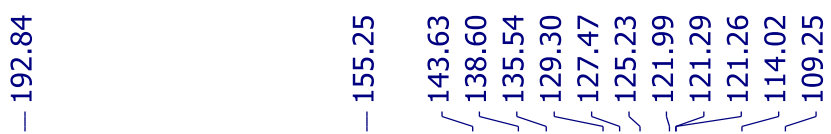

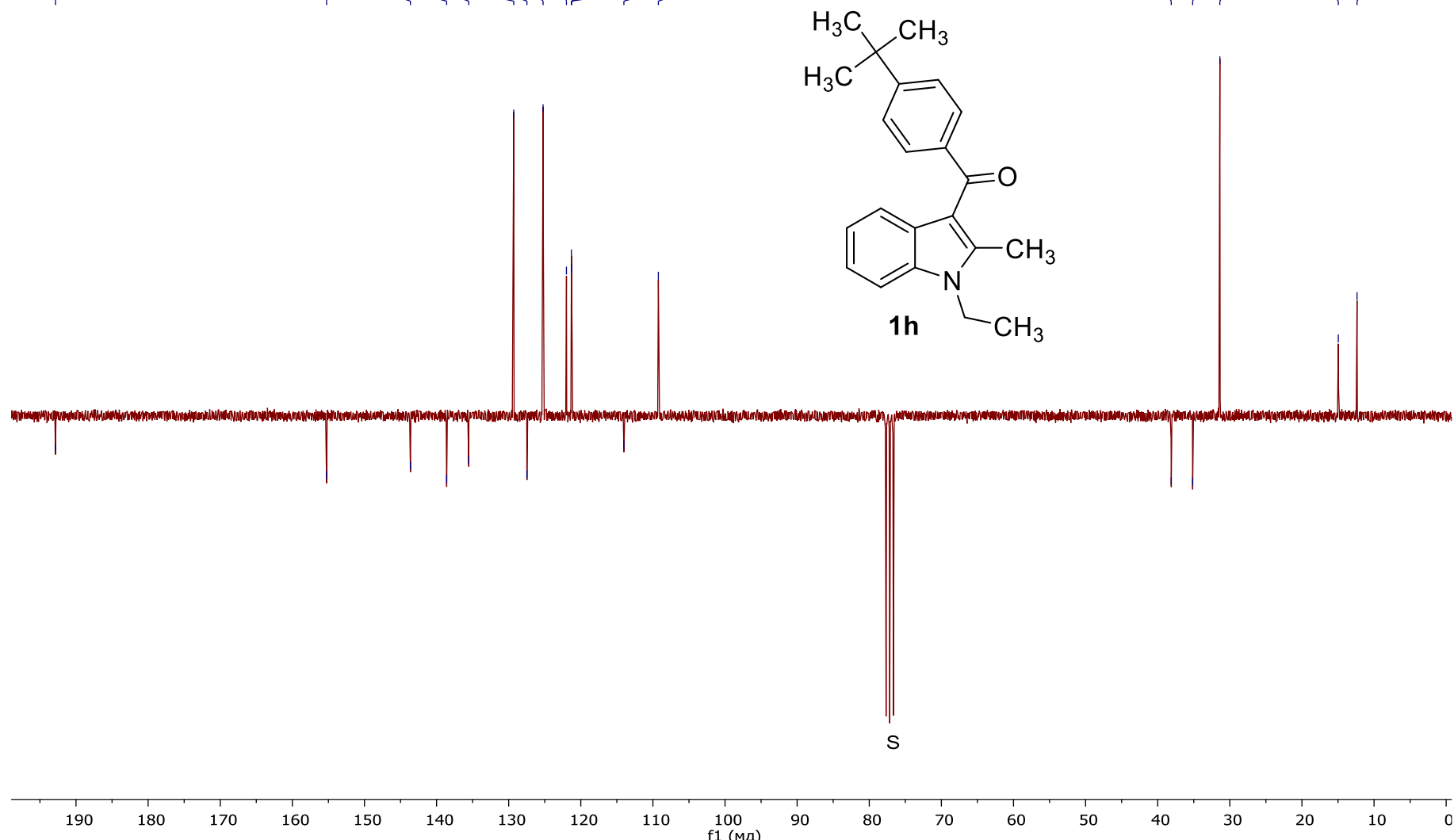

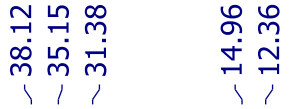


${ }^{1} \mathrm{H} \mathrm{NMR}, \mathrm{CDCl}_{3}, 250 \mathrm{MHz}$

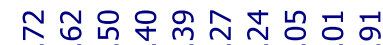

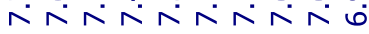

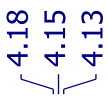

A (t)

4.15

$\mathrm{J}(7.18)$<smiles>Cc1c(C(=O)c2ccccc2)c2ccccc2n1CCCN1CCCC1</smiles>

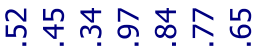

กั่

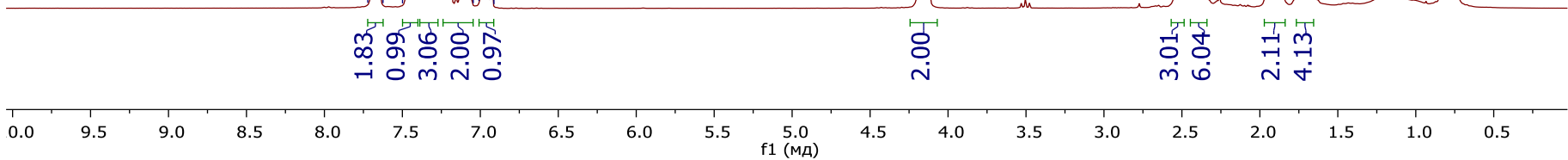

\section{${ }^{13} \mathrm{C}\left\{{ }^{1} \mathrm{H}\right\} \mathrm{NMR}, \mathrm{CDCl}_{3}, 63 \mathrm{MHz}$}

임 $\quad$ 늄

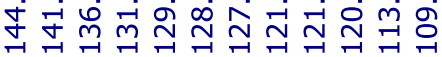

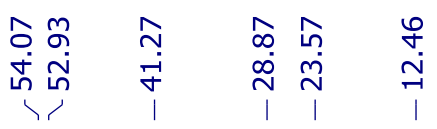

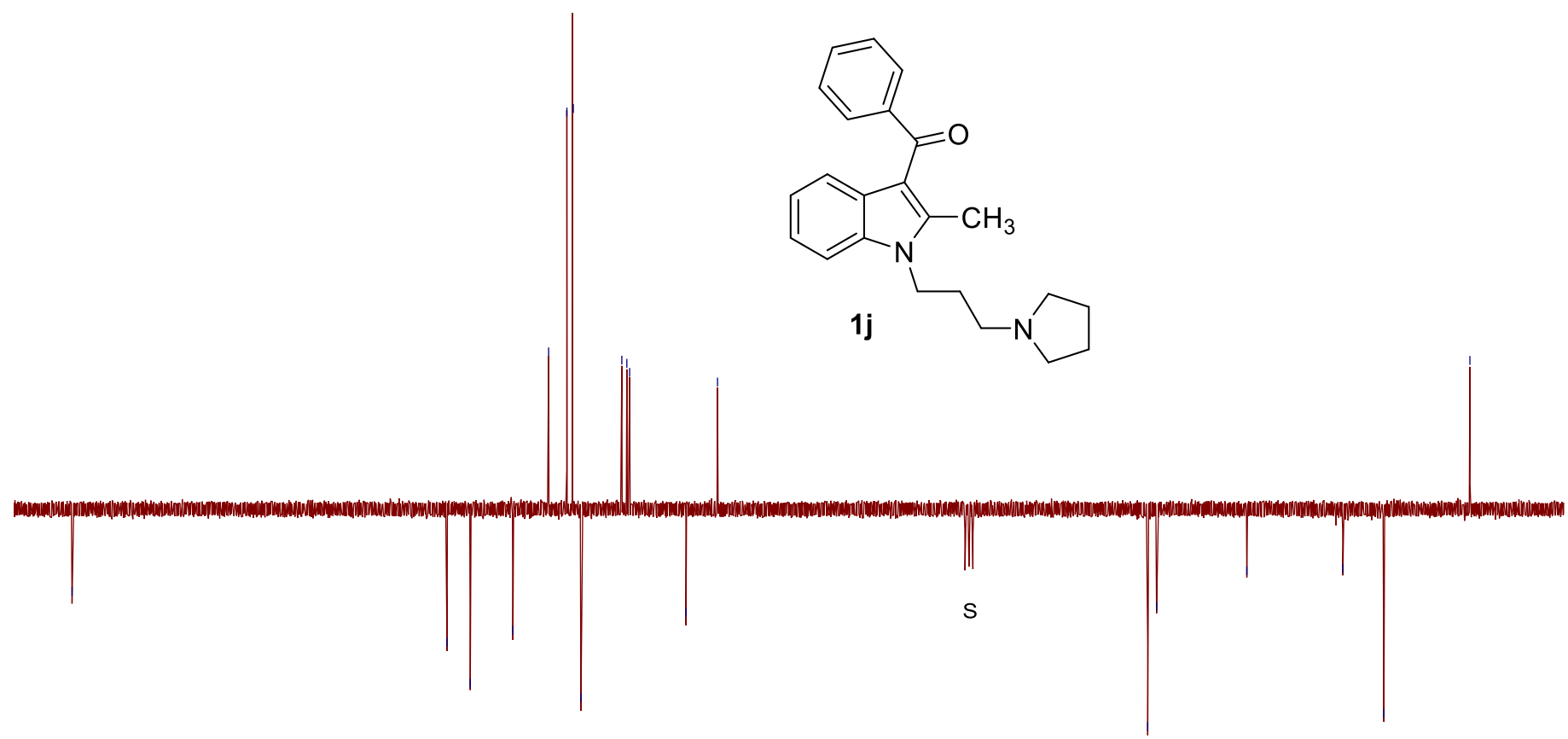




\section{${ }^{1} \mathrm{H} \mathrm{NMR}, \mathrm{CDCl}_{3}, 250 \mathrm{MHz}$}

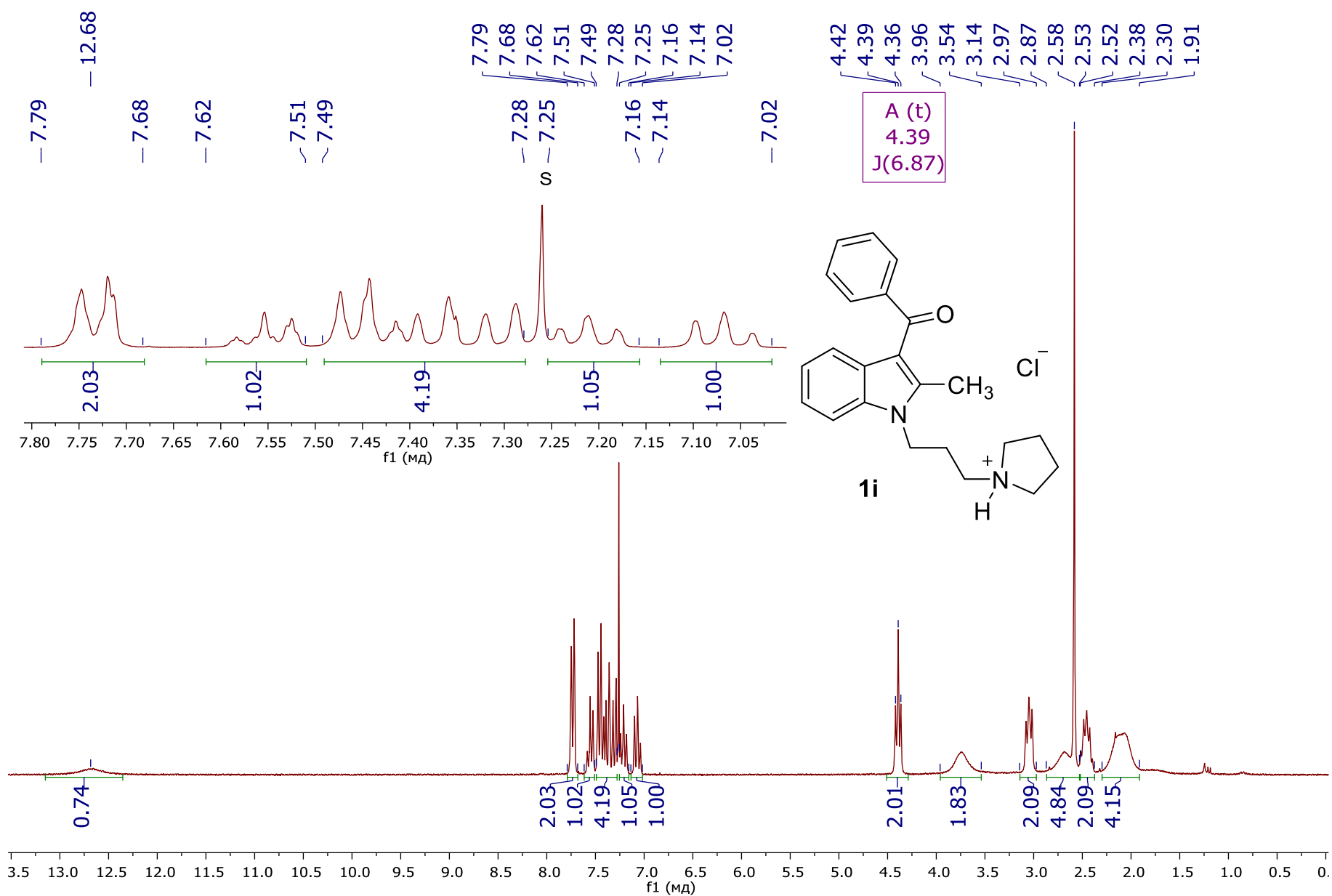

${ }^{13} \mathrm{C}\left\{{ }^{1} \mathrm{H}\right\} \mathrm{NMR}, \mathrm{CDCl}_{3}, 63 \mathrm{MHz}$

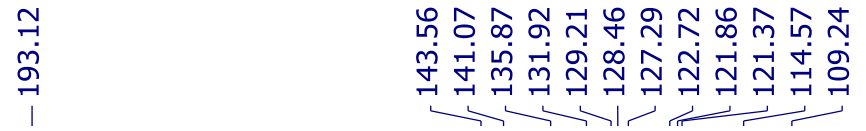

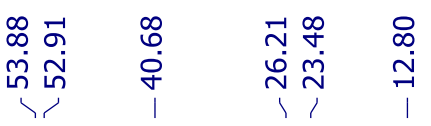

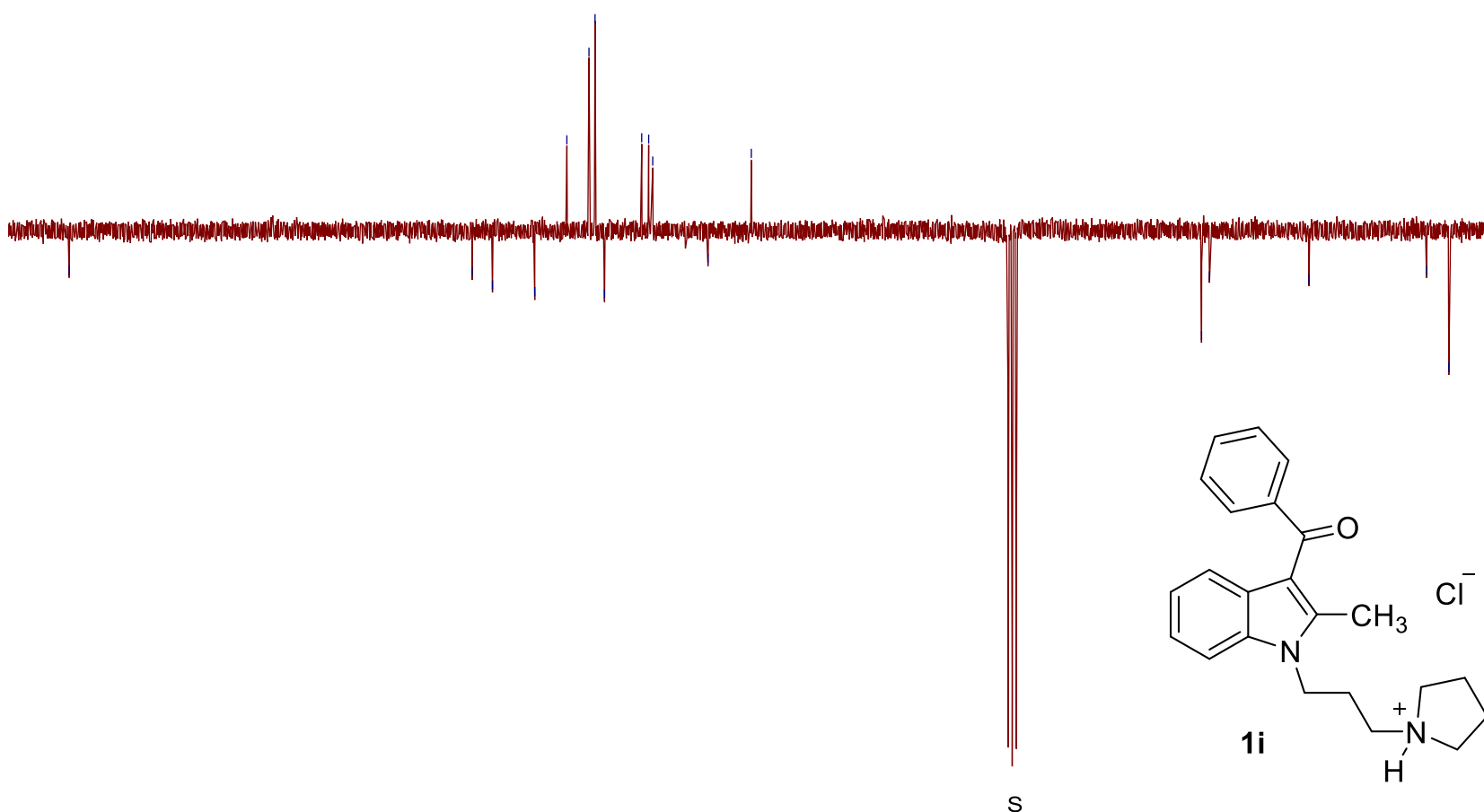

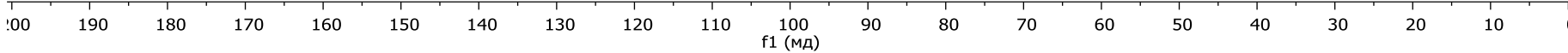


${ }^{1} \mathrm{H} \mathrm{NMR}, \mathrm{CDCl}_{3}, 250 \mathrm{MHz}$

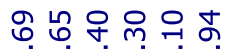

NヘNヘN

$\stackrel{?}{\stackrel{r}{m}}$

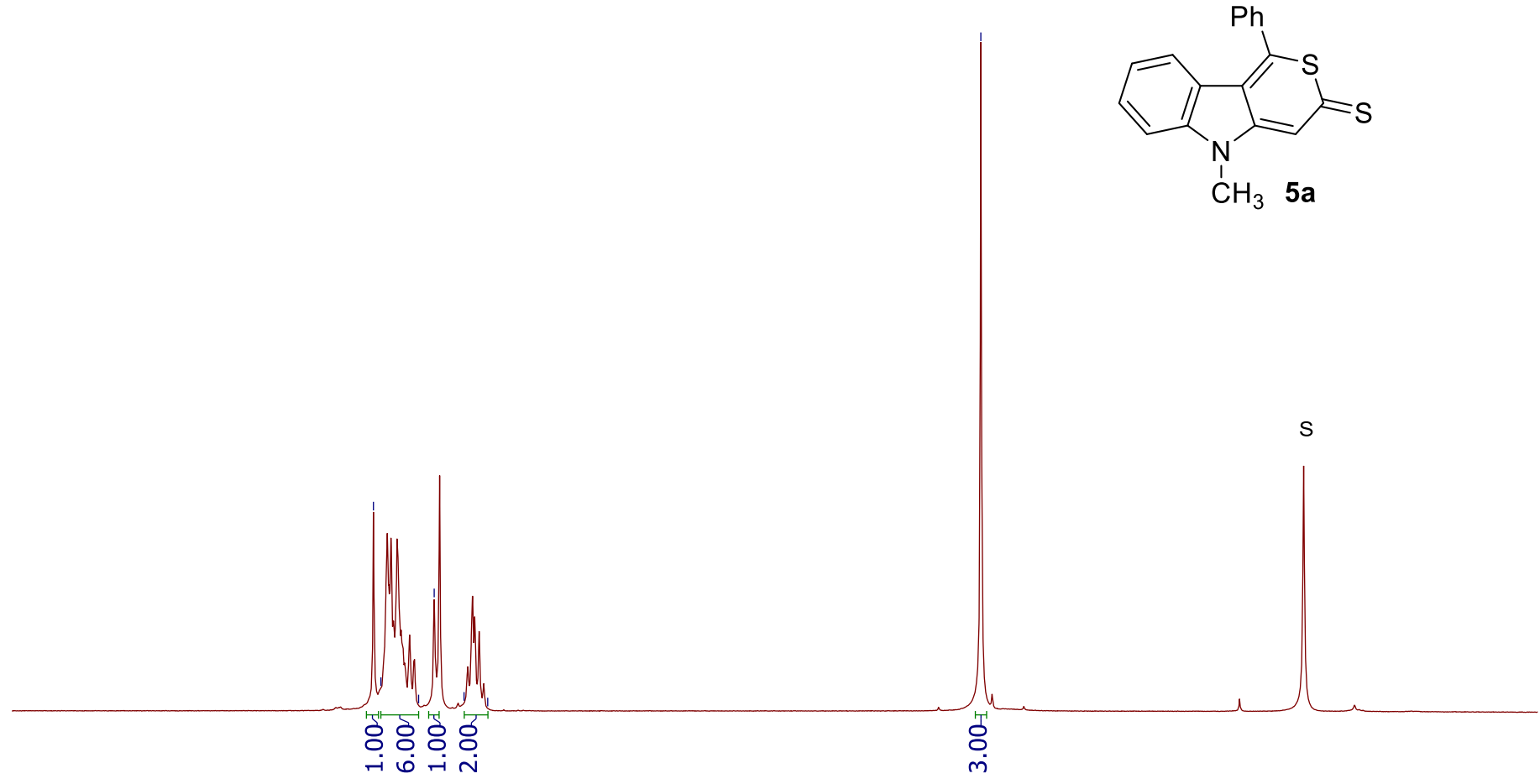

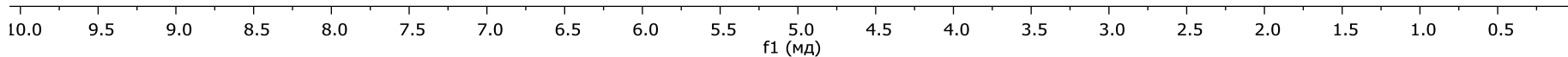

${ }^{13} \mathrm{C}\left\{{ }^{1} \mathrm{H}\right\} \mathrm{NMR}, \mathrm{CDCl}_{3}, 63 \mathrm{MHz}$

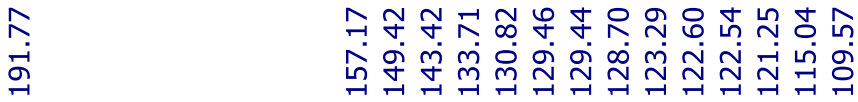

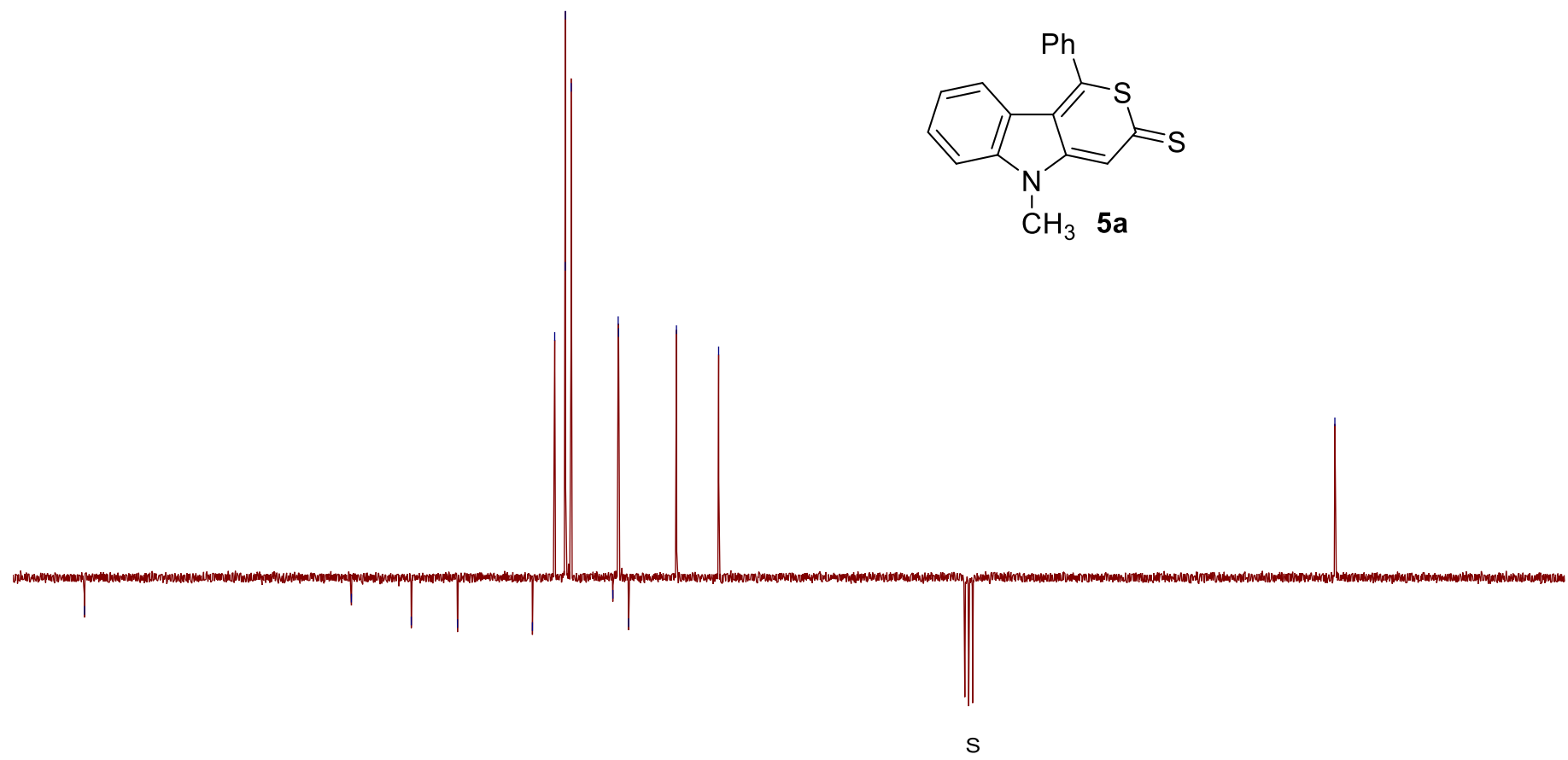

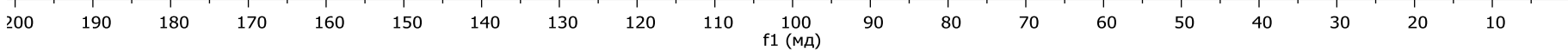


${ }^{1} \mathrm{H} \mathrm{NMR}, \mathrm{CDCl}_{3}, 600 \mathrm{MHz}$

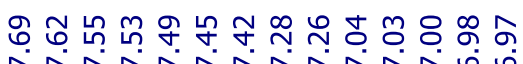

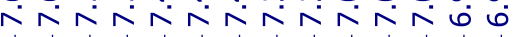

กุ ำ ำ

ํำ ㅇำ ๆ

证西

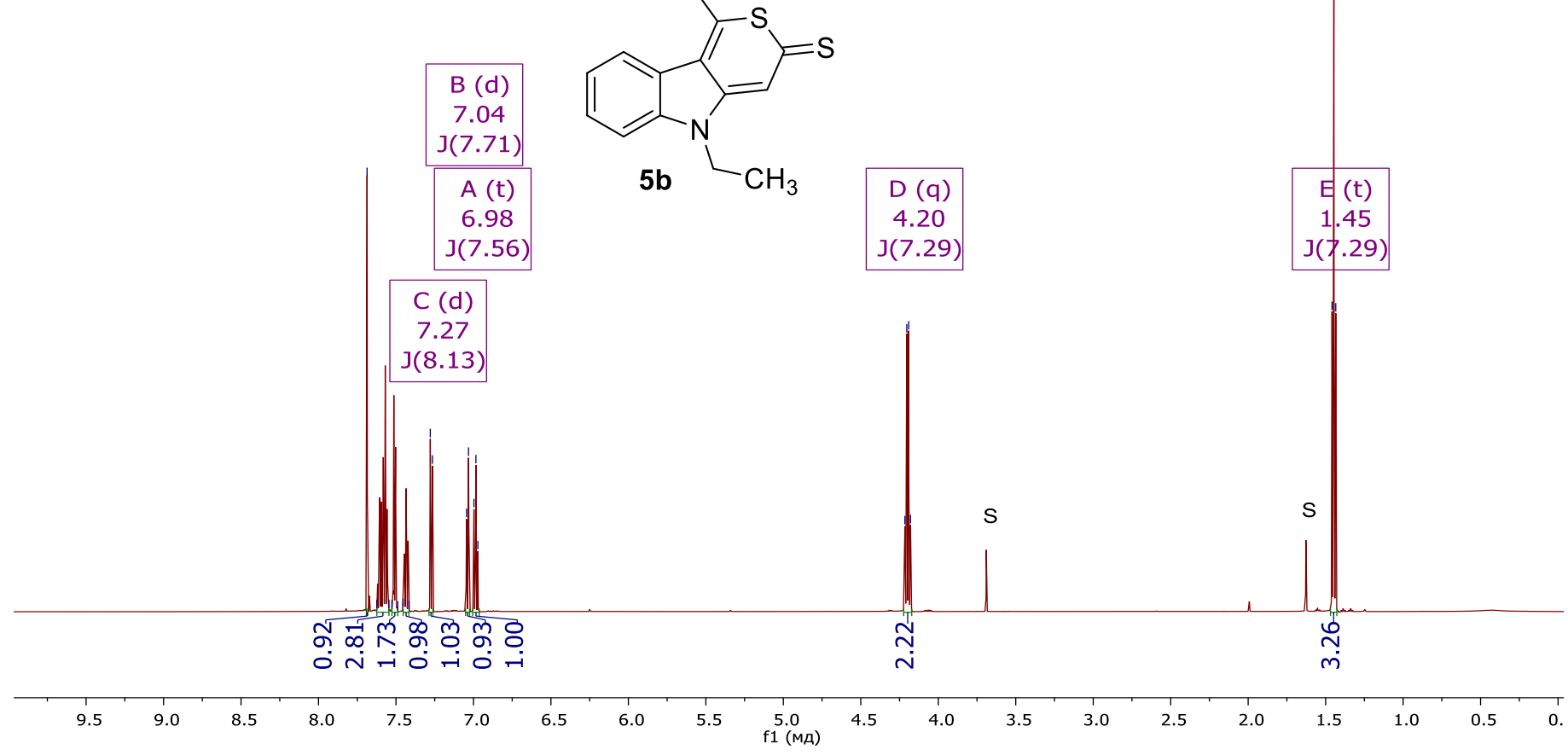

${ }^{13} \mathrm{C}\left\{{ }^{1} \mathrm{H}\right\} \mathrm{NMR}, \mathrm{CDCl}_{3}, 151 \mathrm{MHz}$

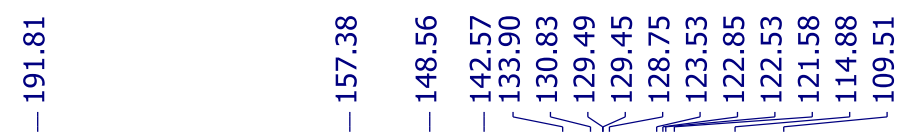
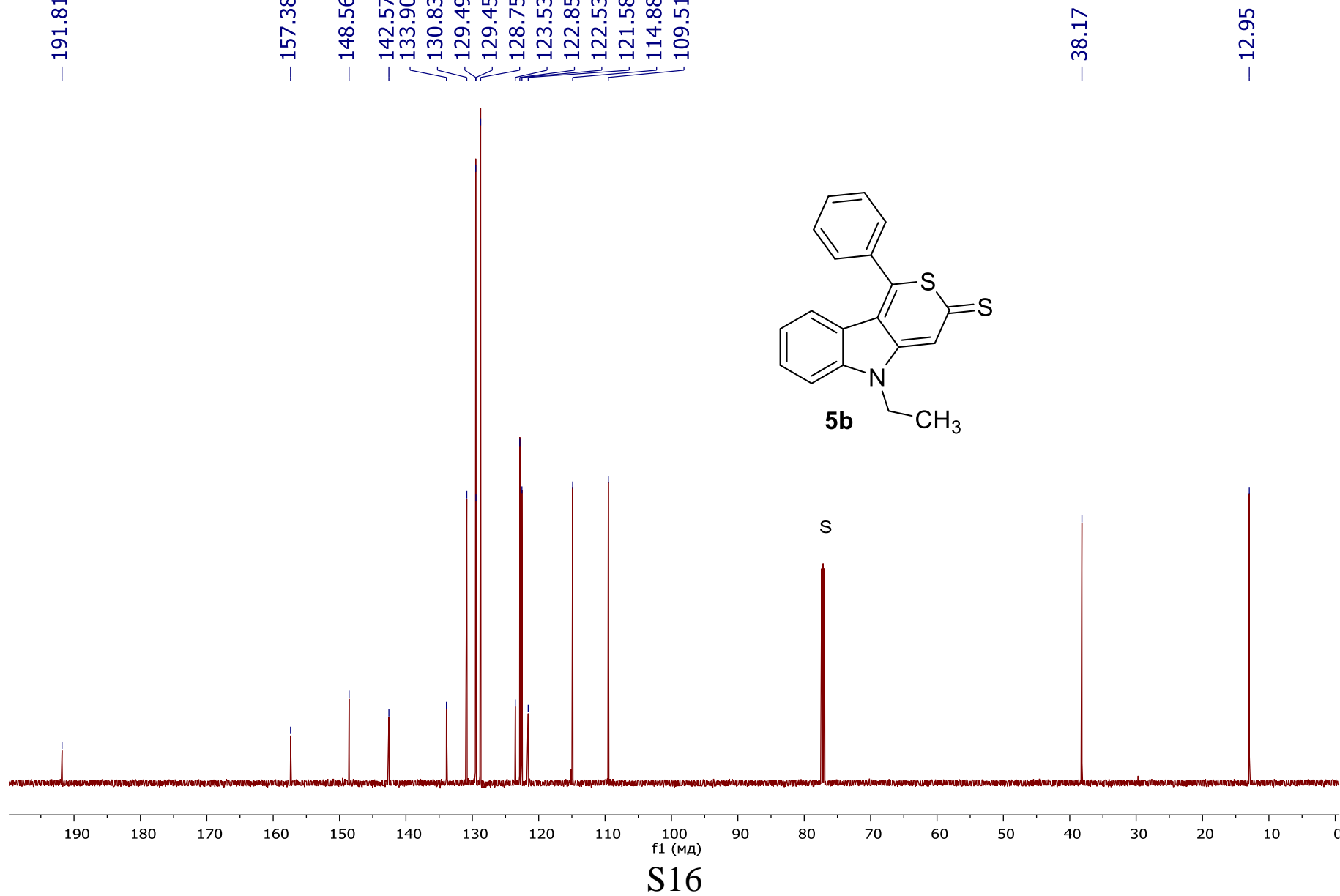


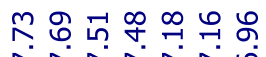

ベNヘNベ゚

$\stackrel{\infty}{m}$

i

เก

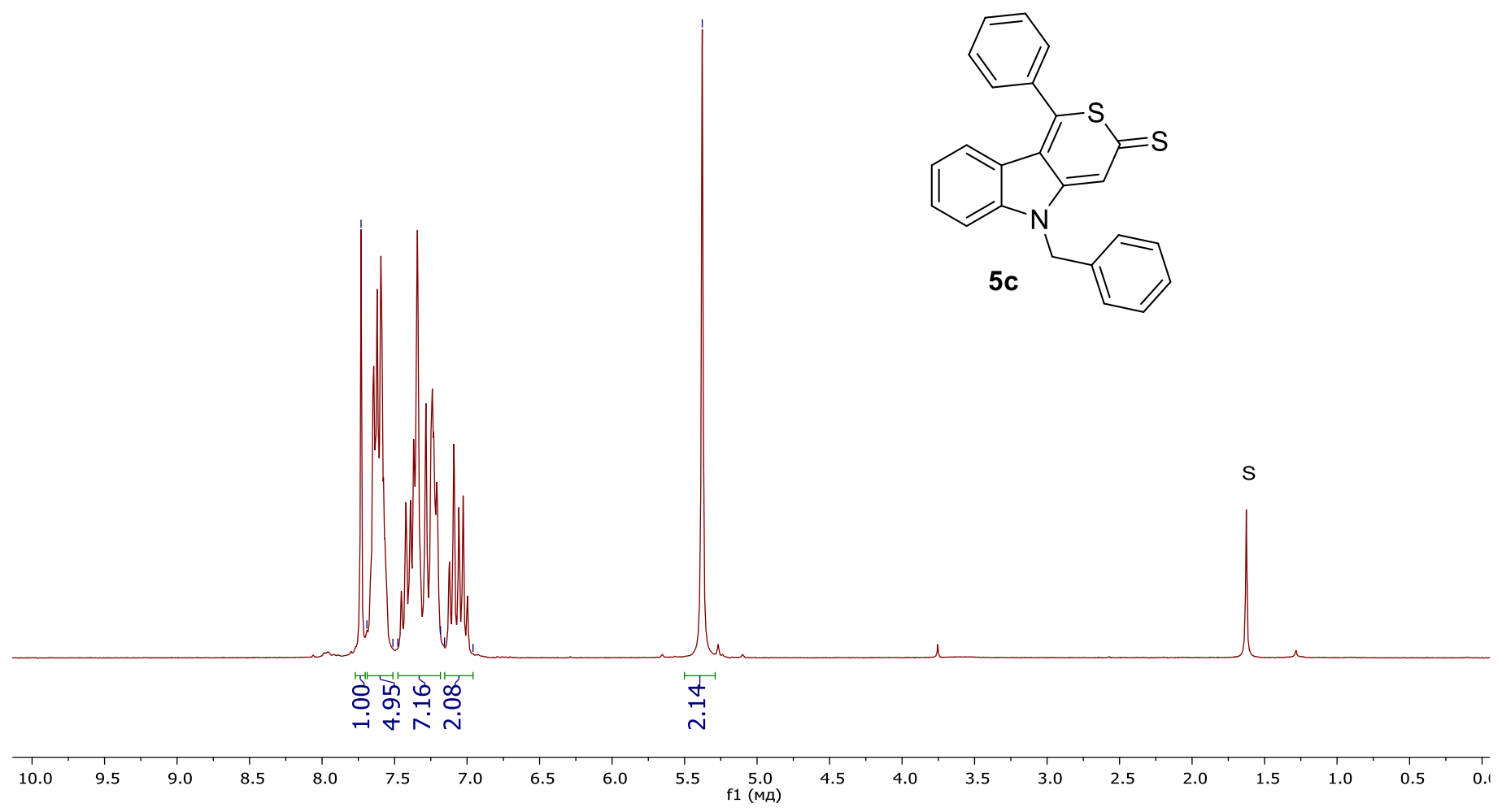

${ }^{13} \mathrm{C}\left\{{ }^{1} \mathrm{H}\right\} \mathrm{NMR}, \mathrm{CDCl}_{3}, 63 \mathrm{MHz}$

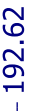

ํำ

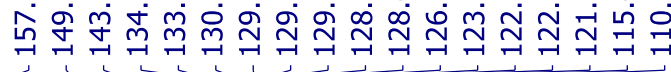
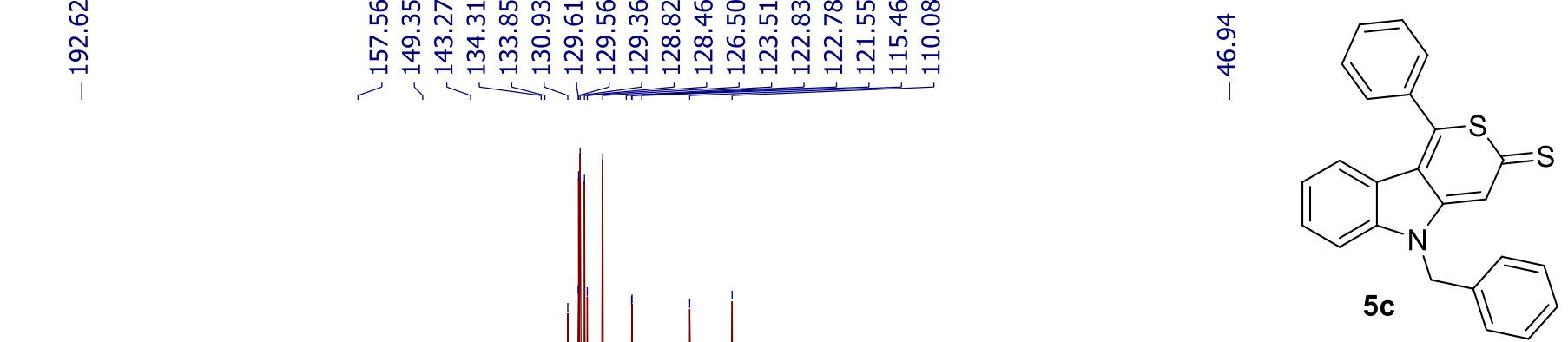

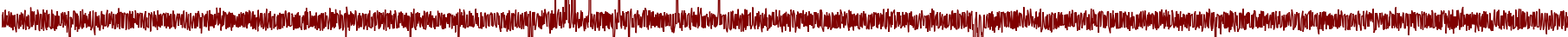

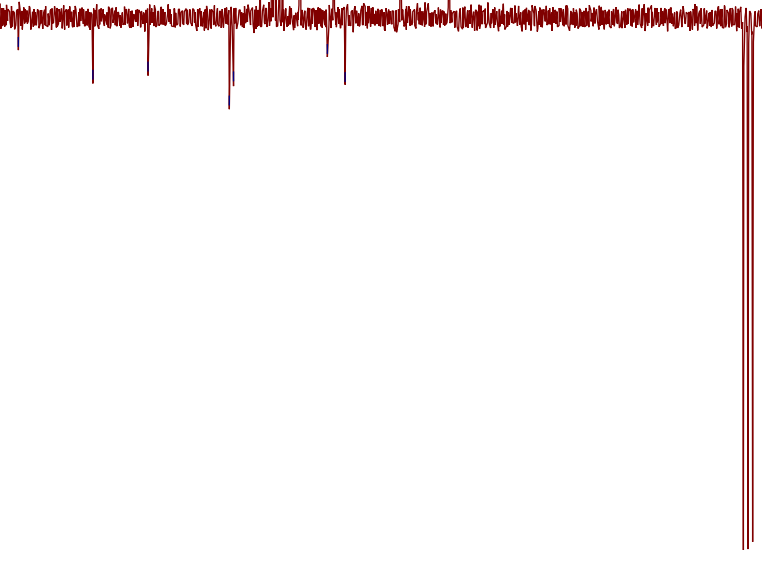

$\mathrm{S}$

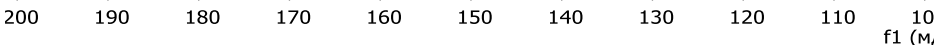




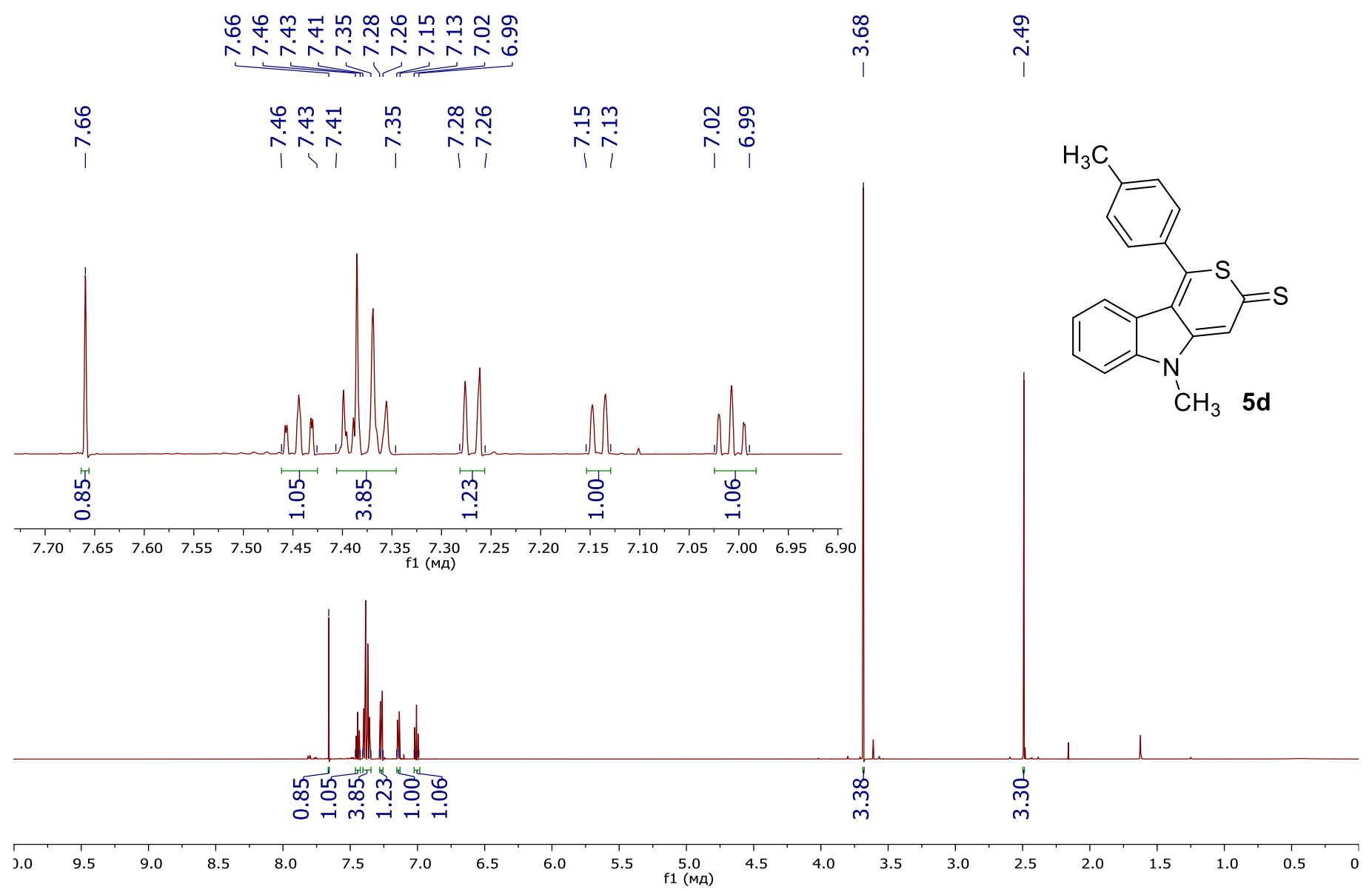

${ }^{13} \mathrm{C}\left\{{ }^{1} \mathrm{H}\right\} \mathrm{NMR}, \mathrm{CDCl}_{3}, 151 \mathrm{MHz}$

م)

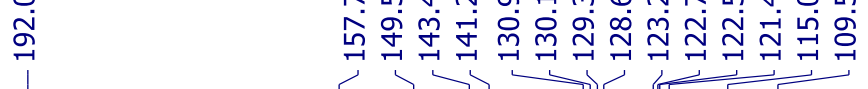

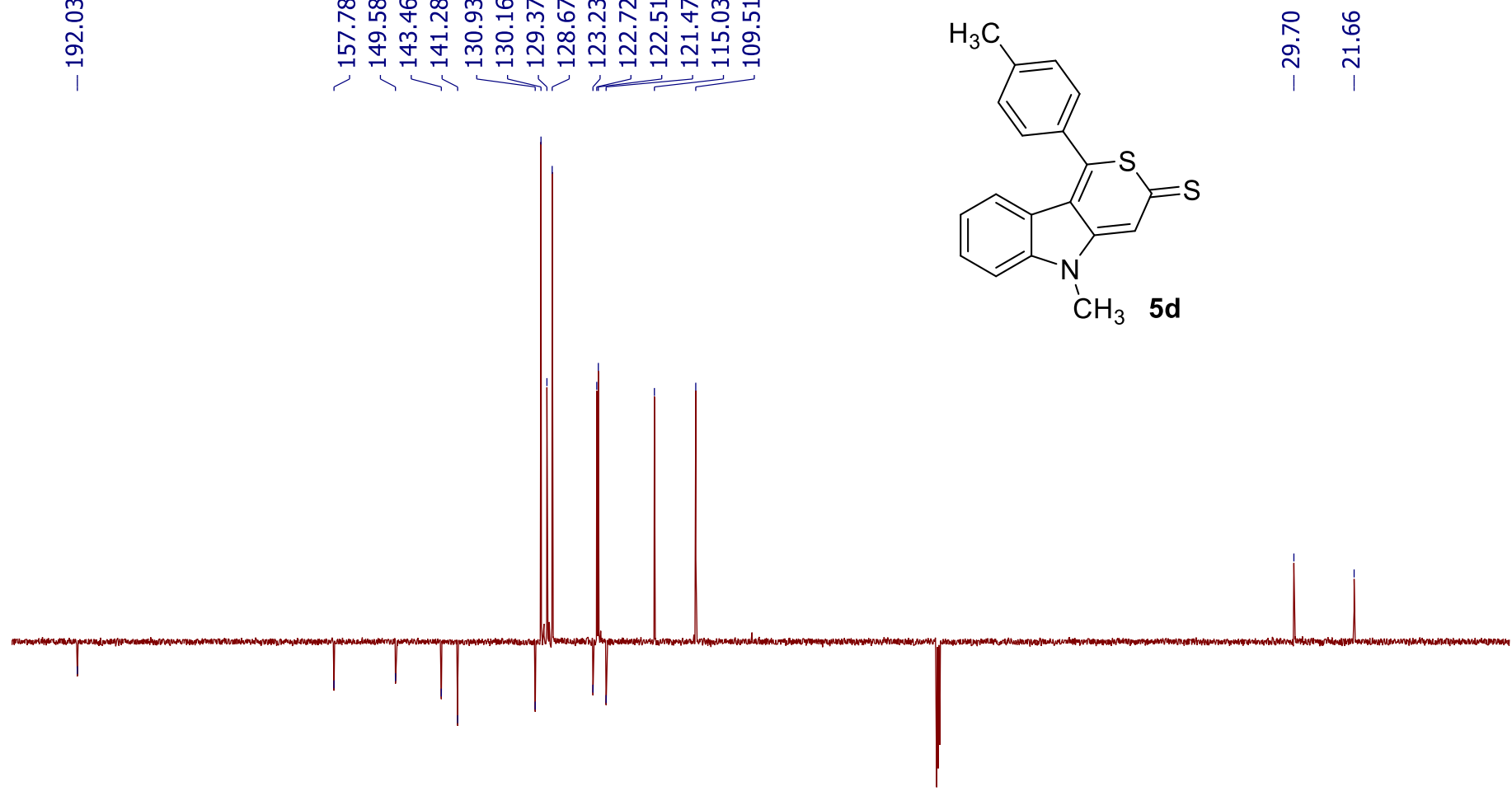

$\mathrm{S}$ 
${ }^{1} \mathrm{H} \mathrm{NMR}, \mathrm{CDCl}_{3}, 250 \mathrm{MHz}$

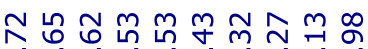

NNNNNNNNG
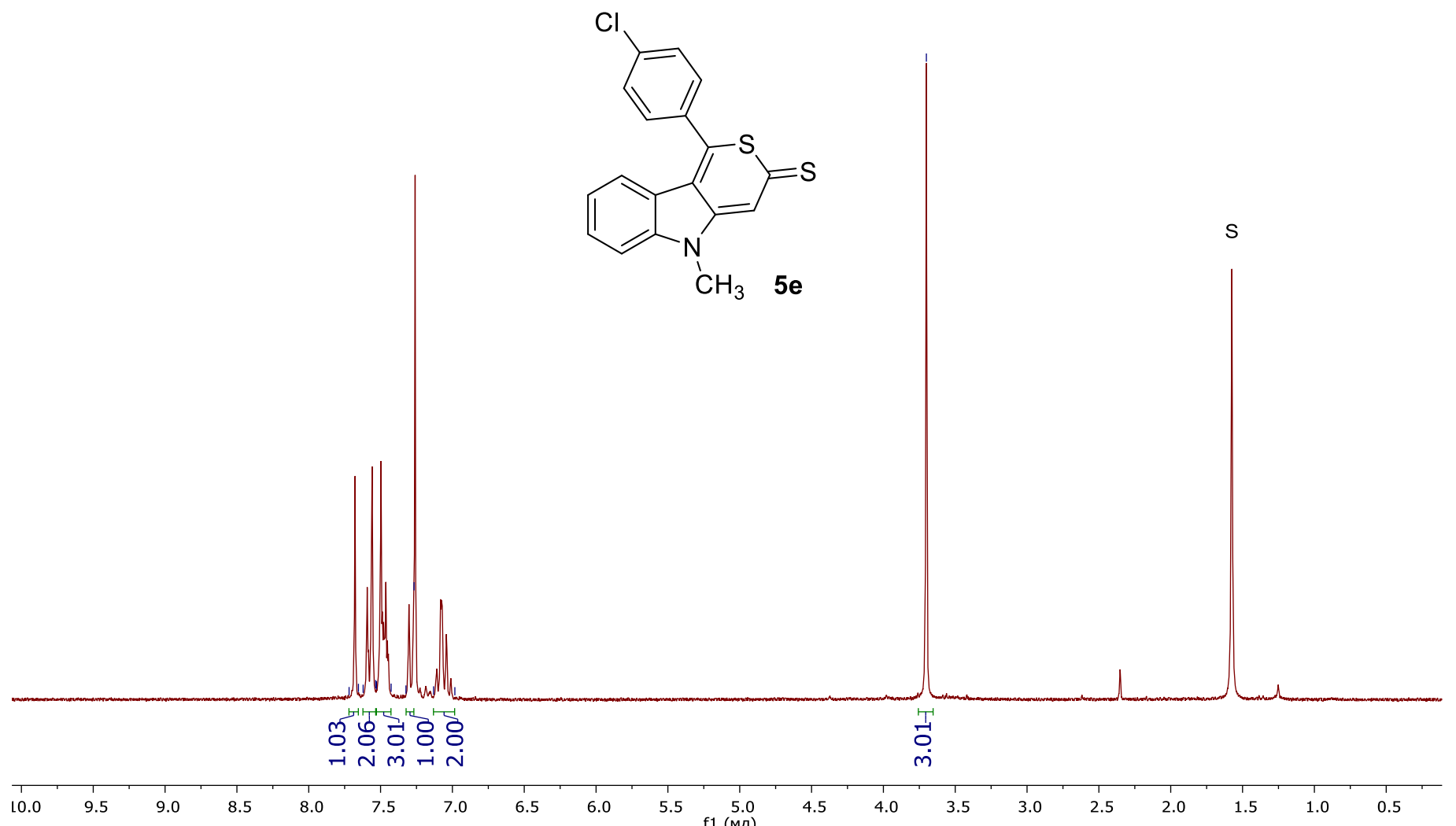

\begin{tabular}{lllllll}
\hline & 9.0 & 8.5 & 8.0 & 7.5 & 7.0
\end{tabular}

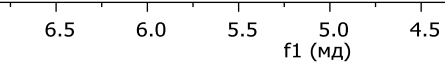

${ }^{13} \mathrm{C}\left\{{ }^{1} \mathrm{H}\right\} \mathrm{NMR}, \mathrm{CDCl}_{3}, 63 \mathrm{MHz}$

รั

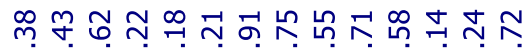

岗守 守

กั่

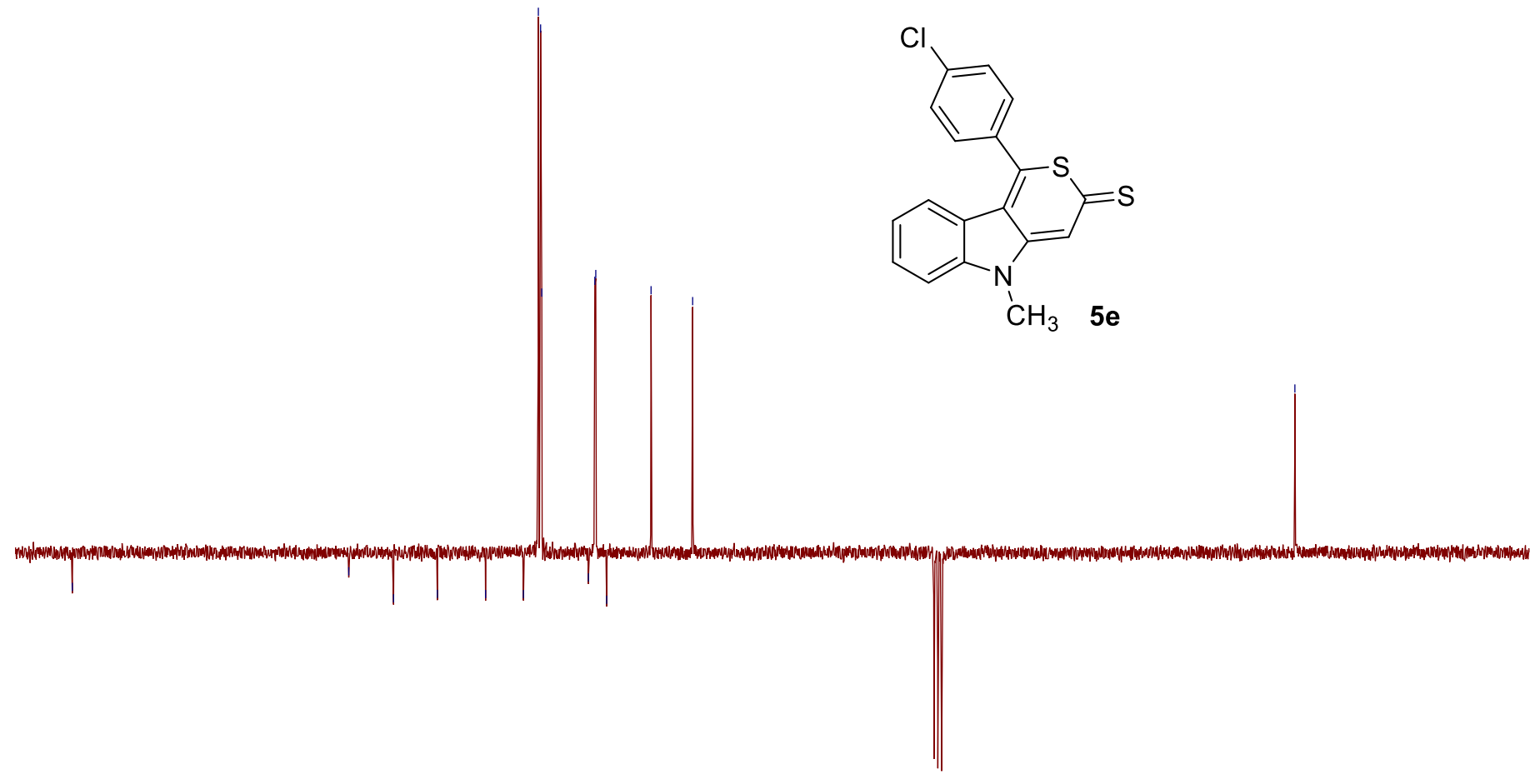

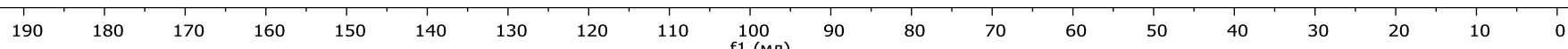


${ }^{1} \mathrm{H} \mathrm{NMR}, \mathrm{CDCl}_{3}, 250 \mathrm{MHz}$

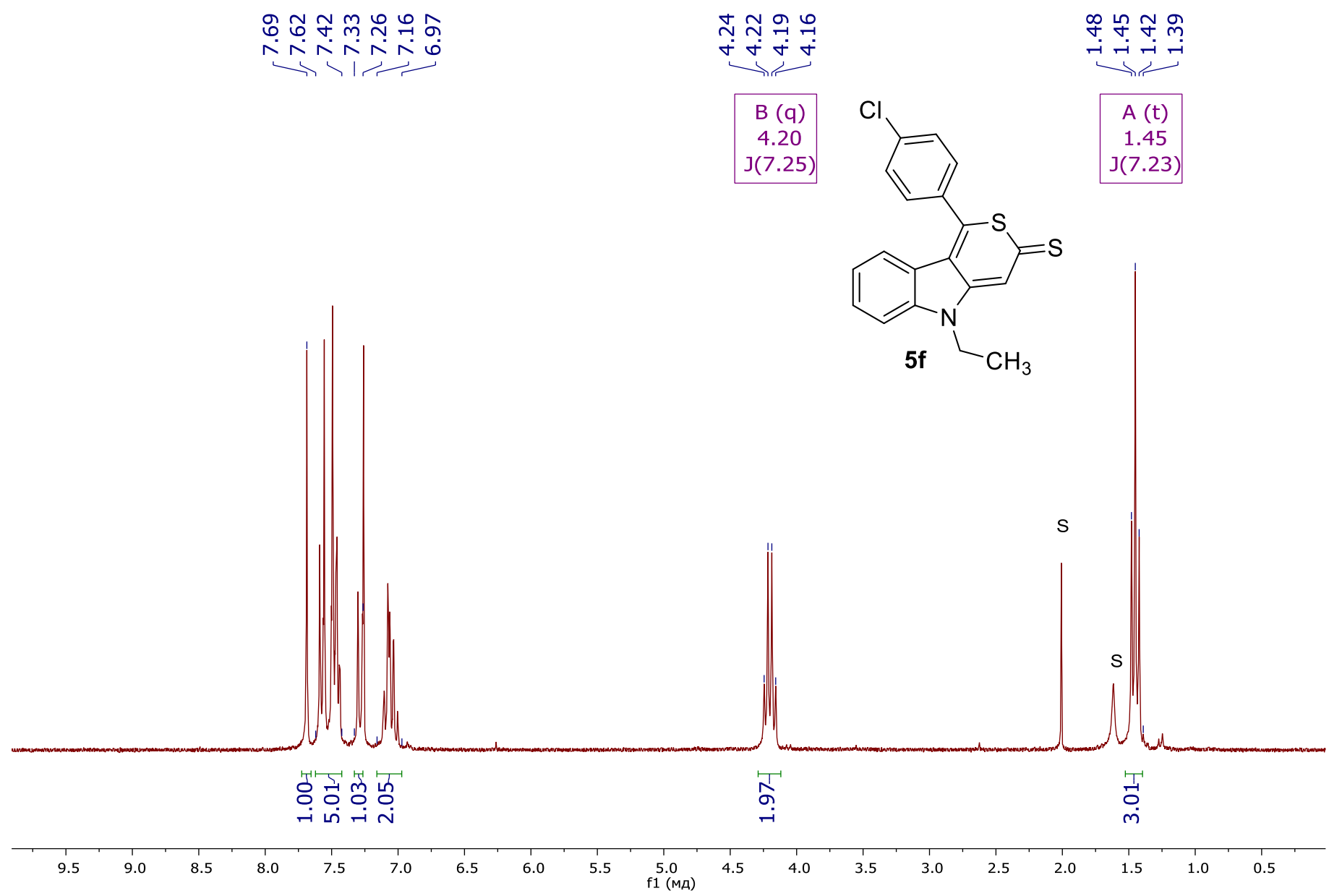

${ }^{13} \mathrm{C}\left\{{ }^{1} \mathrm{H}\right\} \mathrm{NMR}, \mathrm{CDCl}_{3}, 63 \mathrm{MHz}$

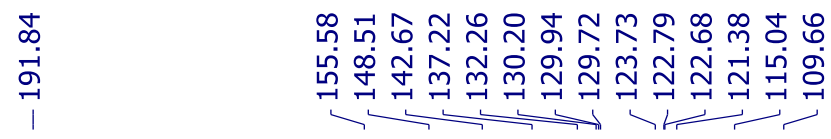
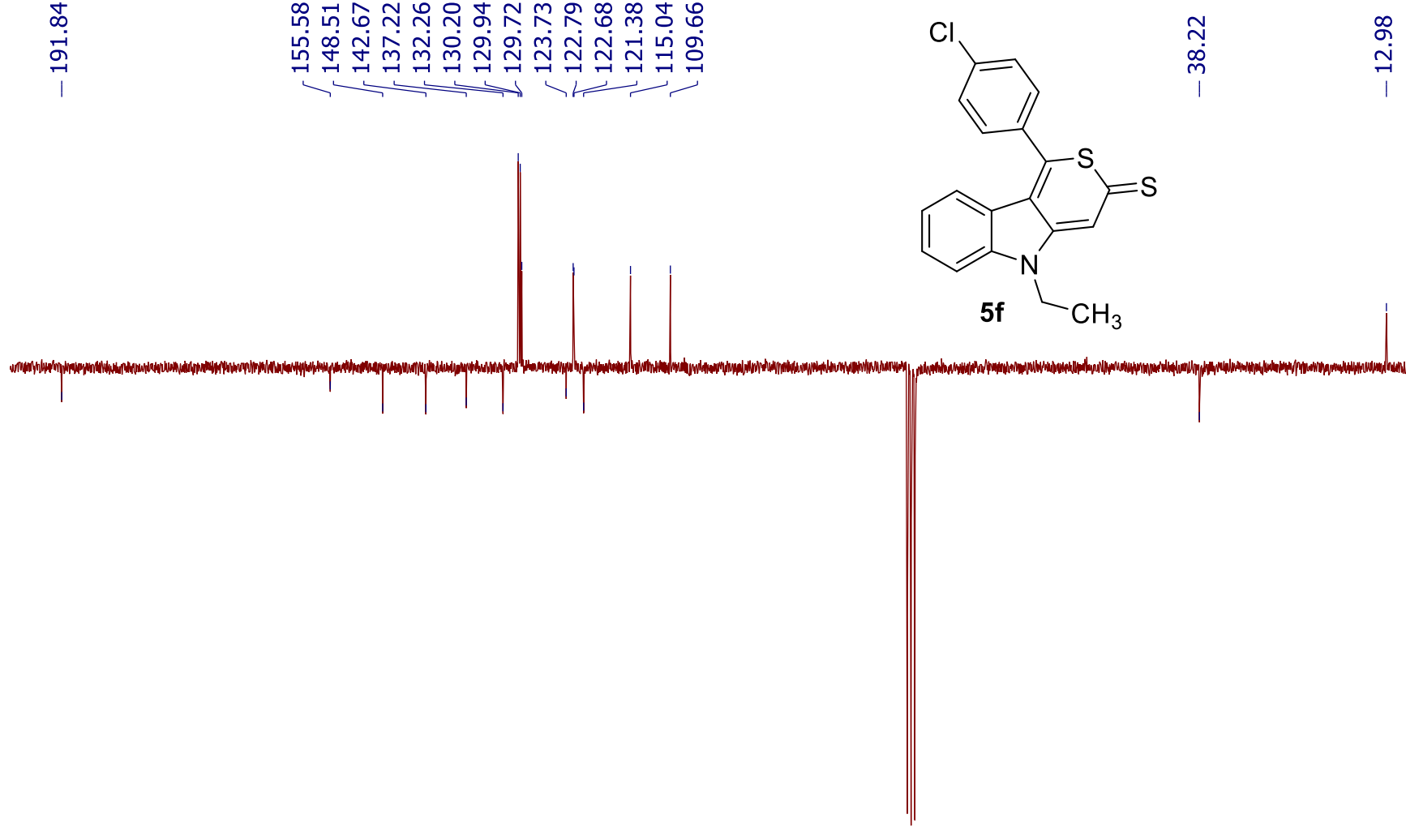

s 
${ }^{1} \mathrm{H} \mathrm{NMR}, \mathrm{CDCl}_{3}, 250 \mathrm{MHz}$

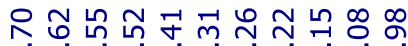

r

$\stackrel{+}{\stackrel{4}{*}}$

師

S
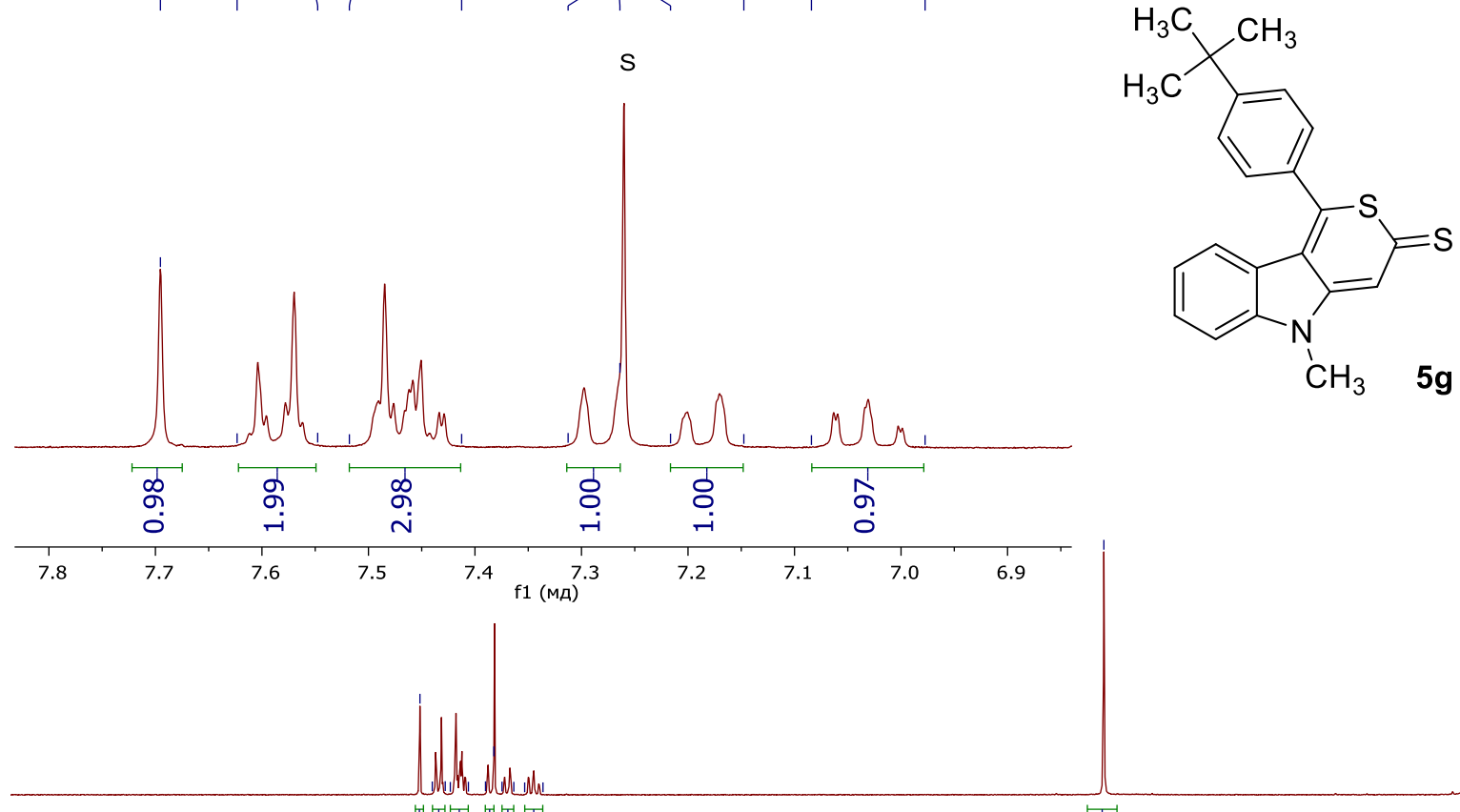

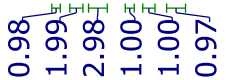

i̊

\section{궁}

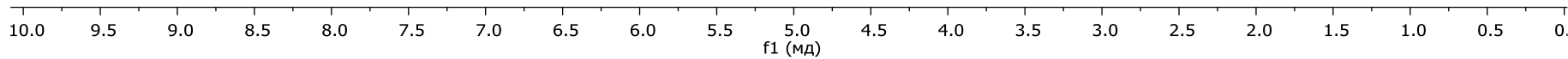

${ }^{13} \mathrm{C}\left\{{ }^{1} \mathrm{H}\right\} \mathrm{NMR}, \mathrm{CDCl}_{3}, 63 \mathrm{MHz}$

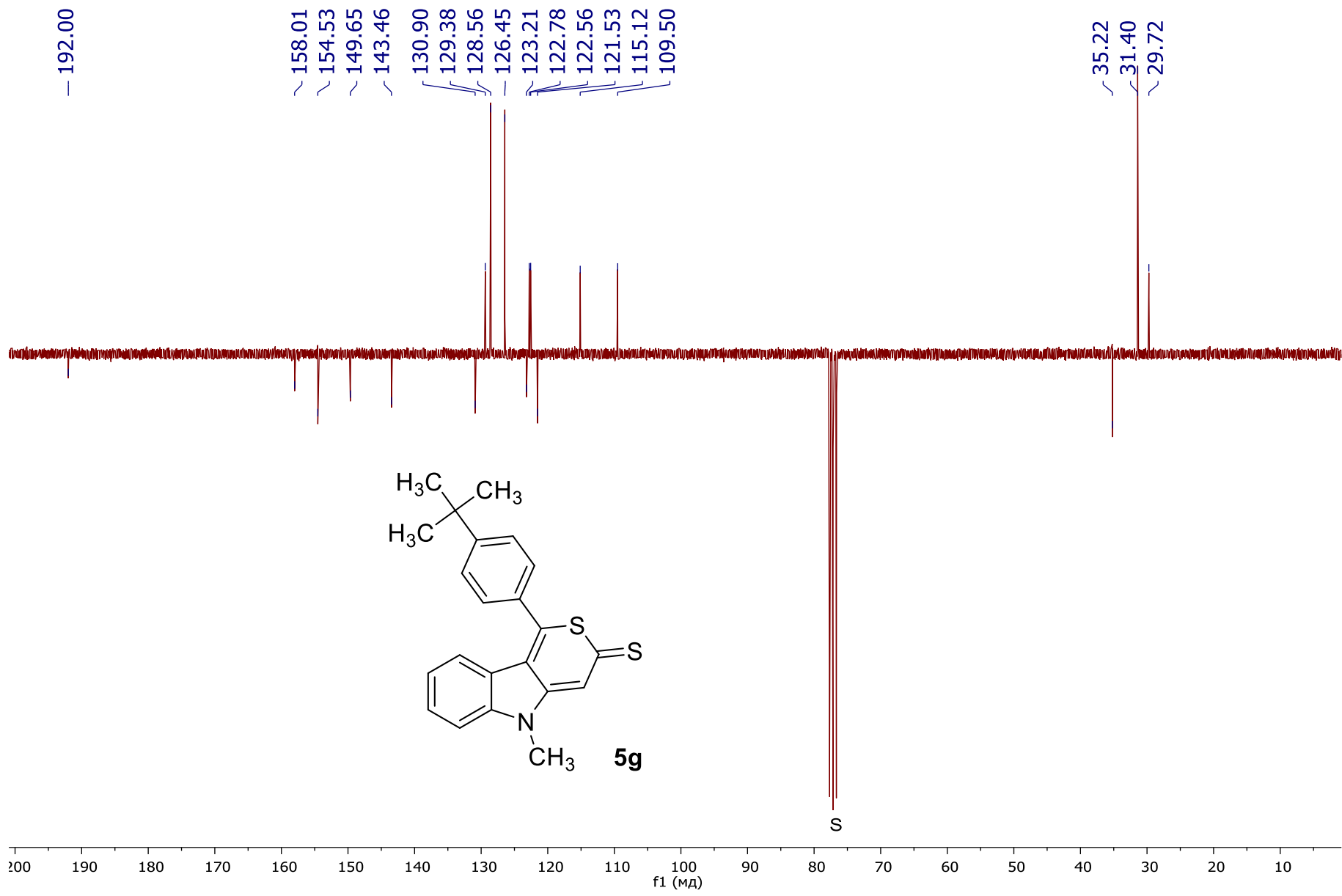


${ }^{1} \mathrm{H} \mathrm{NMR}, \mathrm{CDCl}_{3}, 250 \mathrm{MHz}$

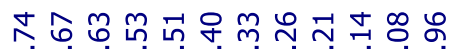

NNNヘNNNヘN゙

N

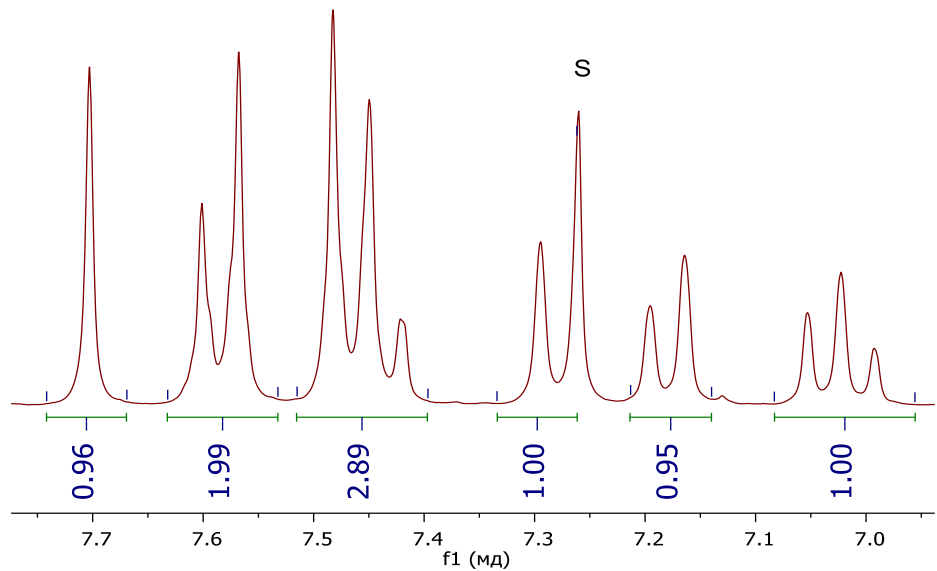

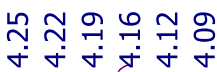

ֻ̊

A (q)

4.21

$\mathrm{J}(7.24)$

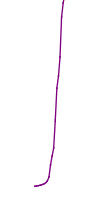

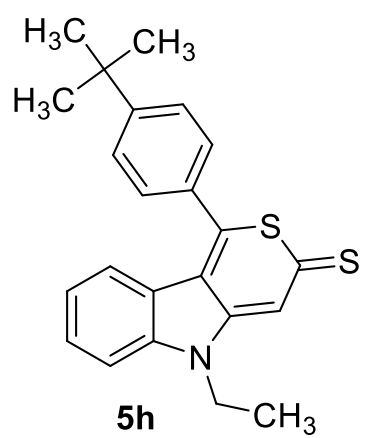

นั่

$\mathrm{CH}_{3}$

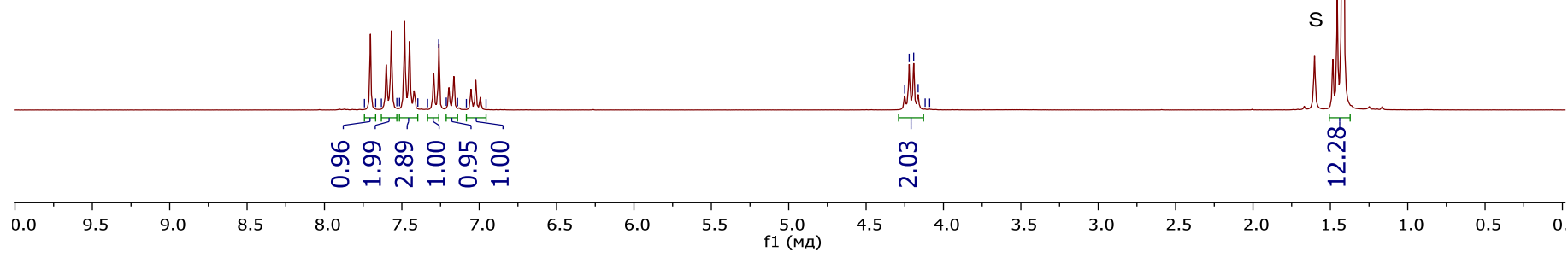

${ }^{13} \mathrm{C}\left\{{ }^{1} \mathrm{H}\right\} \mathrm{NMR}, \mathrm{CDCl}_{3}, 63 \mathrm{MHz}$

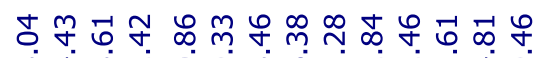

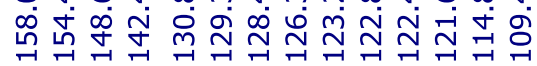

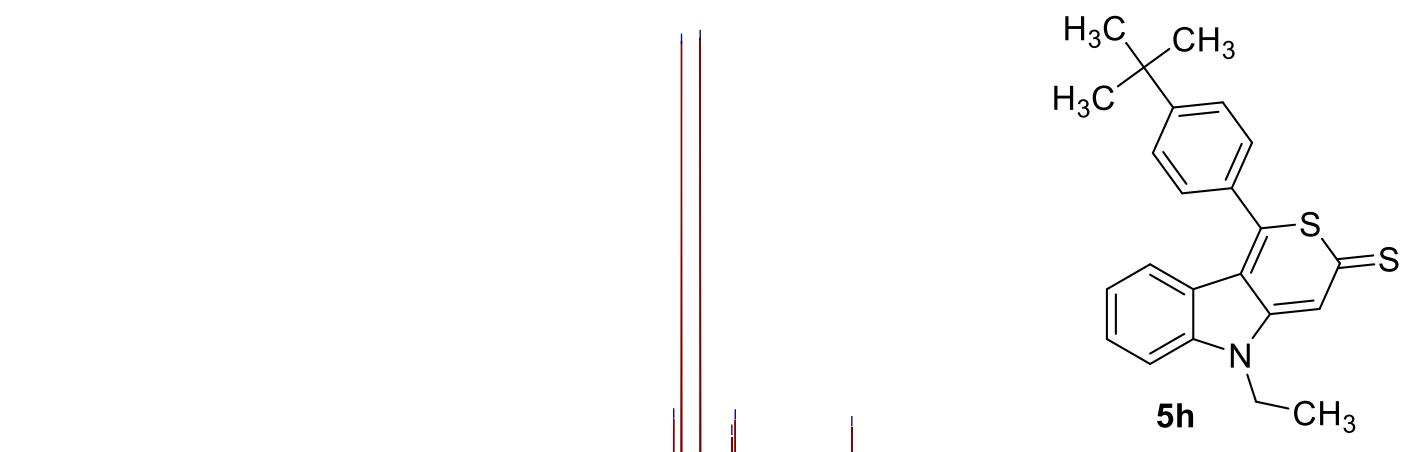

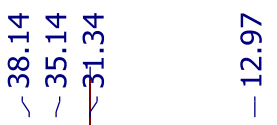

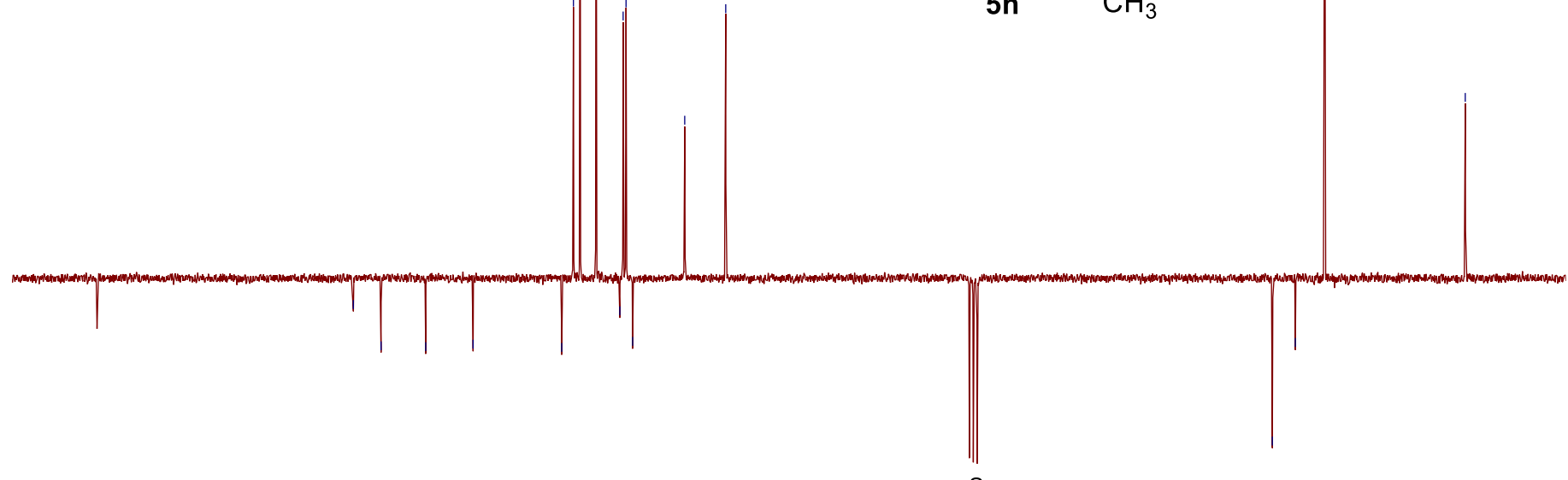

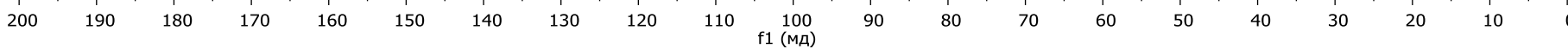


${ }^{1} \mathrm{H} \mathrm{NMR}, \mathrm{CDCl}_{3}, 250 \mathrm{MHz}$

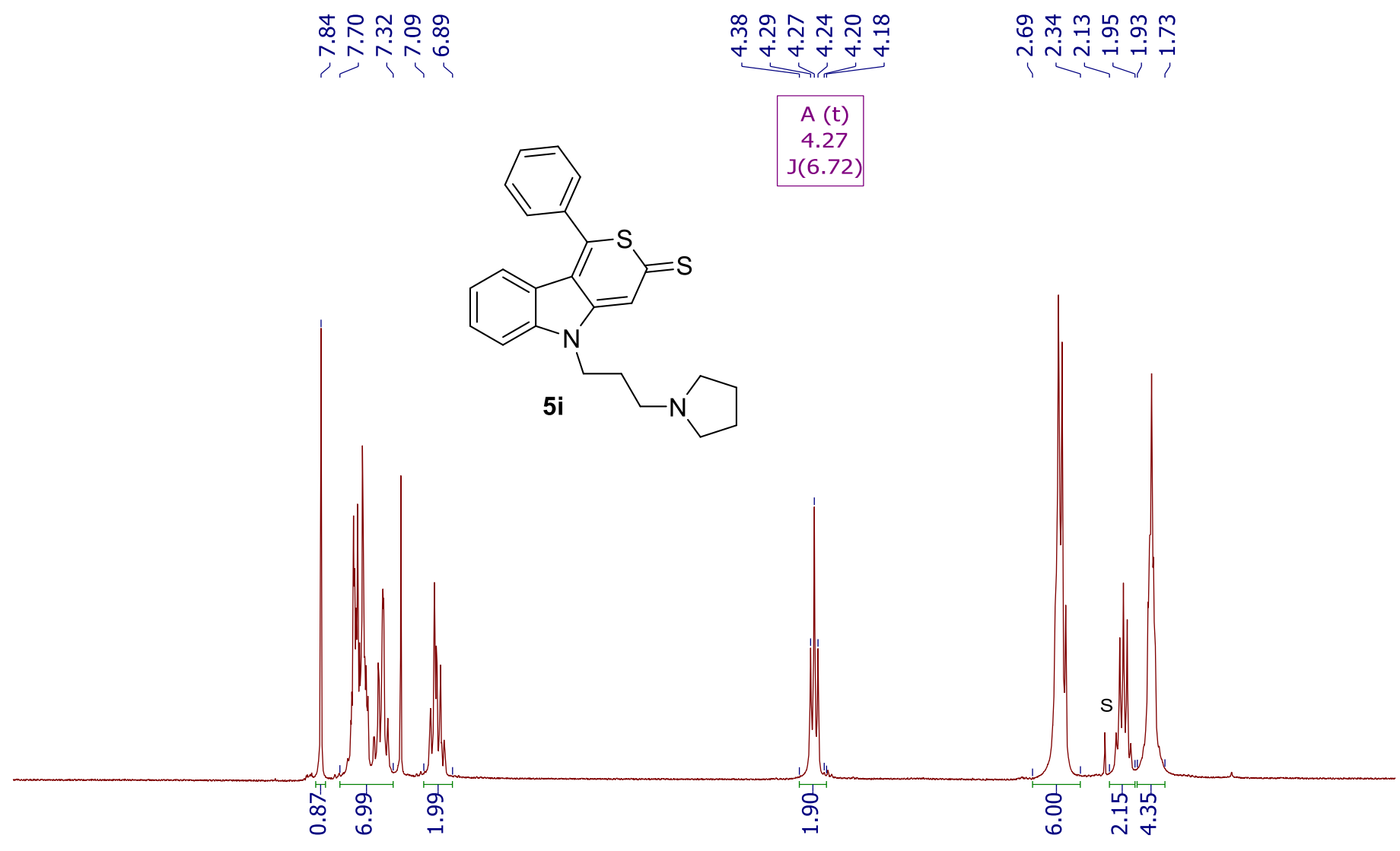

\begin{tabular}{|c|c|c|c|c|c|c|c|c|c|c|c|c|c|c|c|c|c|c|c|}
\hline 10.0 & 9.5 & 9.0 & 8.5 & 8.0 & 7.5 & 7.0 & 6.5 & 6.0 & 5.5 & $\begin{array}{r}5.0 \\
\text { f1 }(\text { (MA) }\end{array}$ & 4.5 & 4.0 & 3.5 & 3.0 & 2.5 & 2.0 & 1.5 & 1.0 & 0.5 \\
\hline
\end{tabular}

${ }^{13} \mathrm{C}\left\{{ }^{1} \mathrm{H}\right\} \mathrm{NMR}, \mathrm{CDCl}_{3}, 63 \mathrm{MHz}$

$\underset{\substack{- \\ \text { - }}}{\stackrel{\text { I }}{1}}$

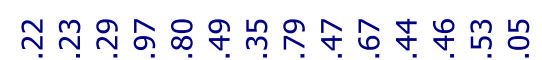

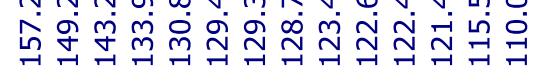

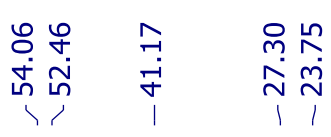

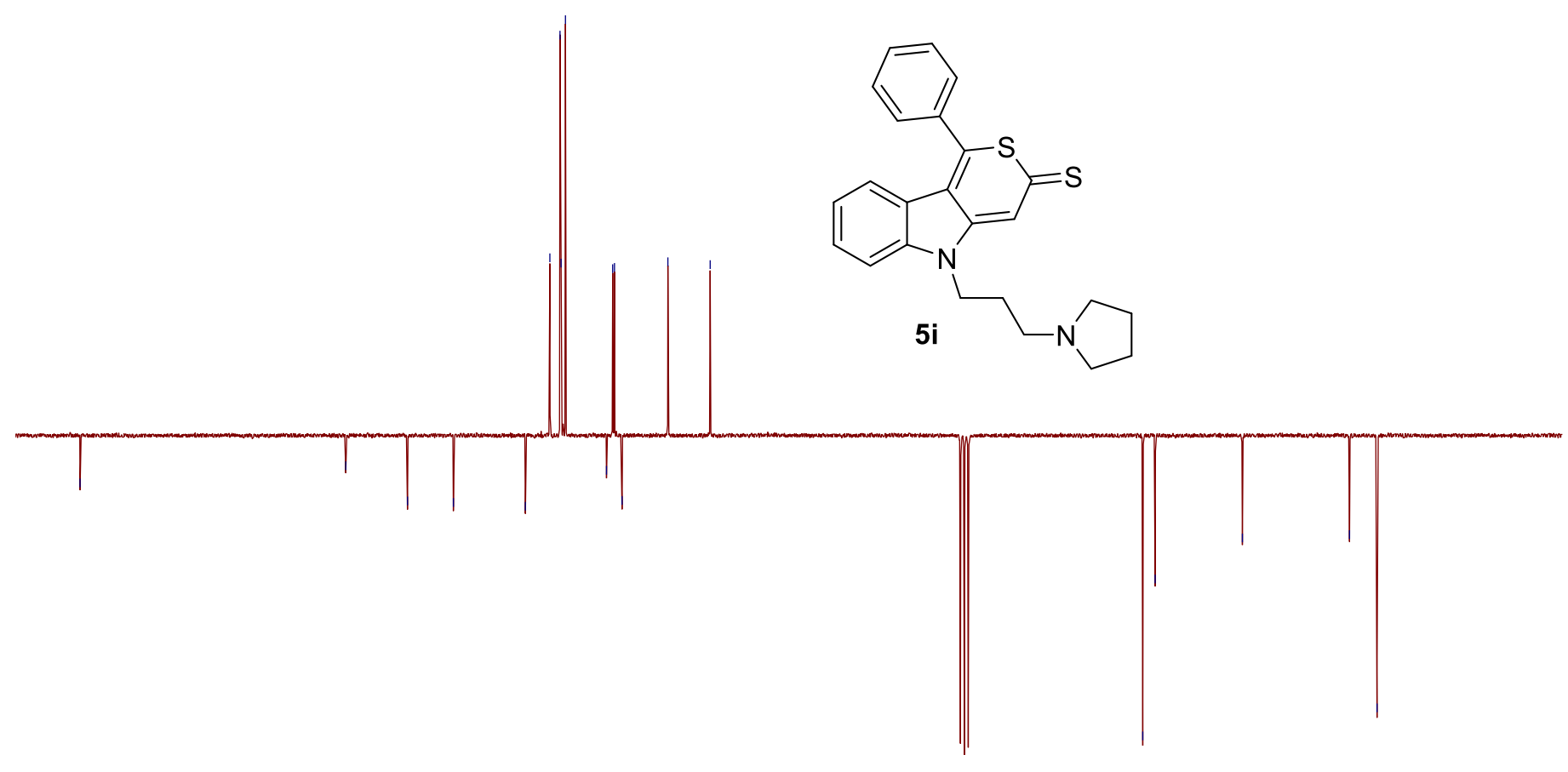

s

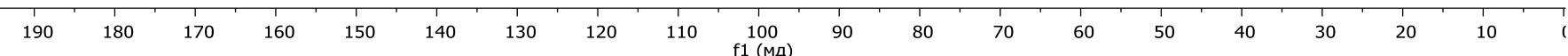




\section{CALCULATIONS DATA}

Figure S1. The attractive forces in system $\mathbf{2 4 a}$ according to AIM calculations. $\rho\left(r_{\mathrm{c}}\right)-$ electron density values.

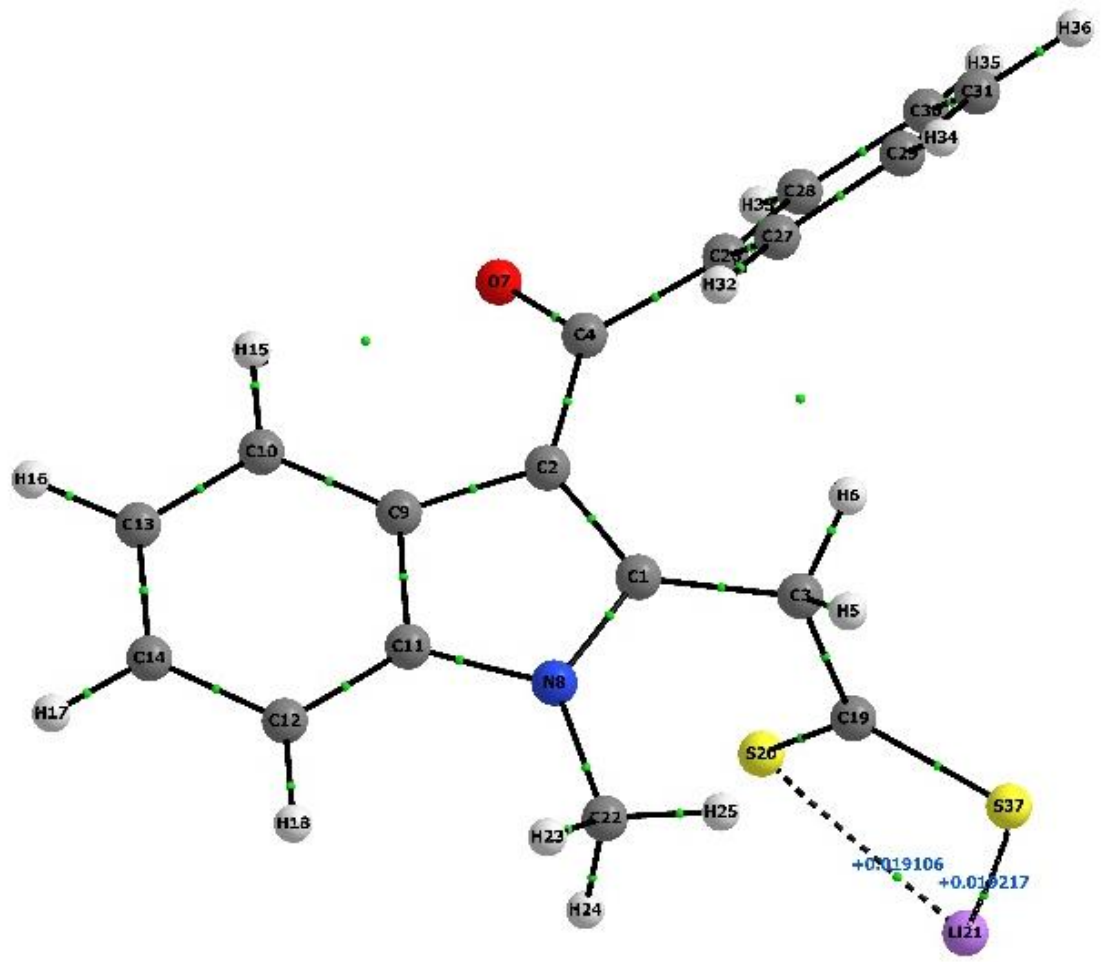

Figure S2. The attractive forces in system $\mathbf{2 4} \mathbf{a}^{\prime}$ according to AIM calculations. $\rho\left(r_{\mathrm{c}}\right)-$ electron density values.

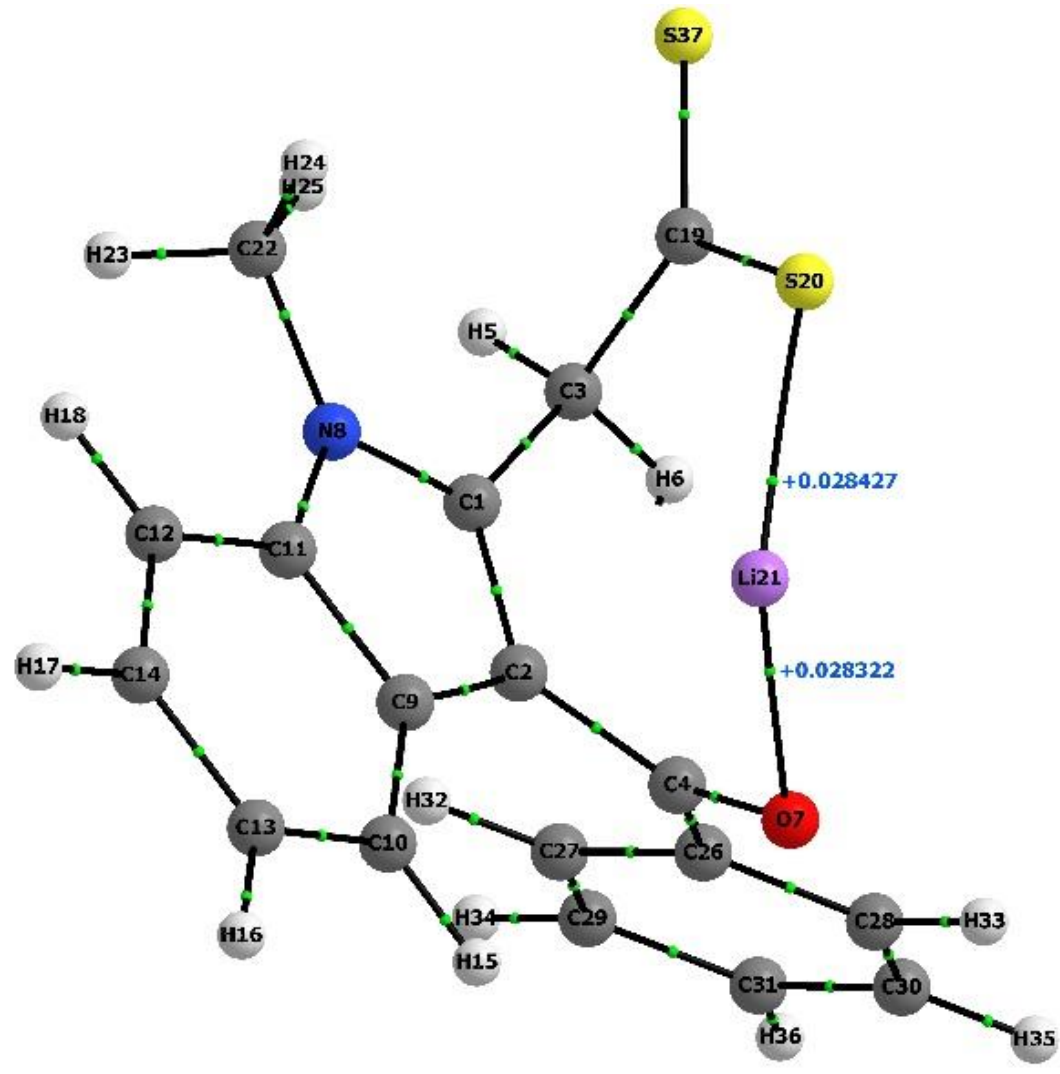


Figure S3. The attractive forces in system 23a according to AIM calculations. $\rho\left(r_{\mathrm{c}}\right)$ - electron density values.

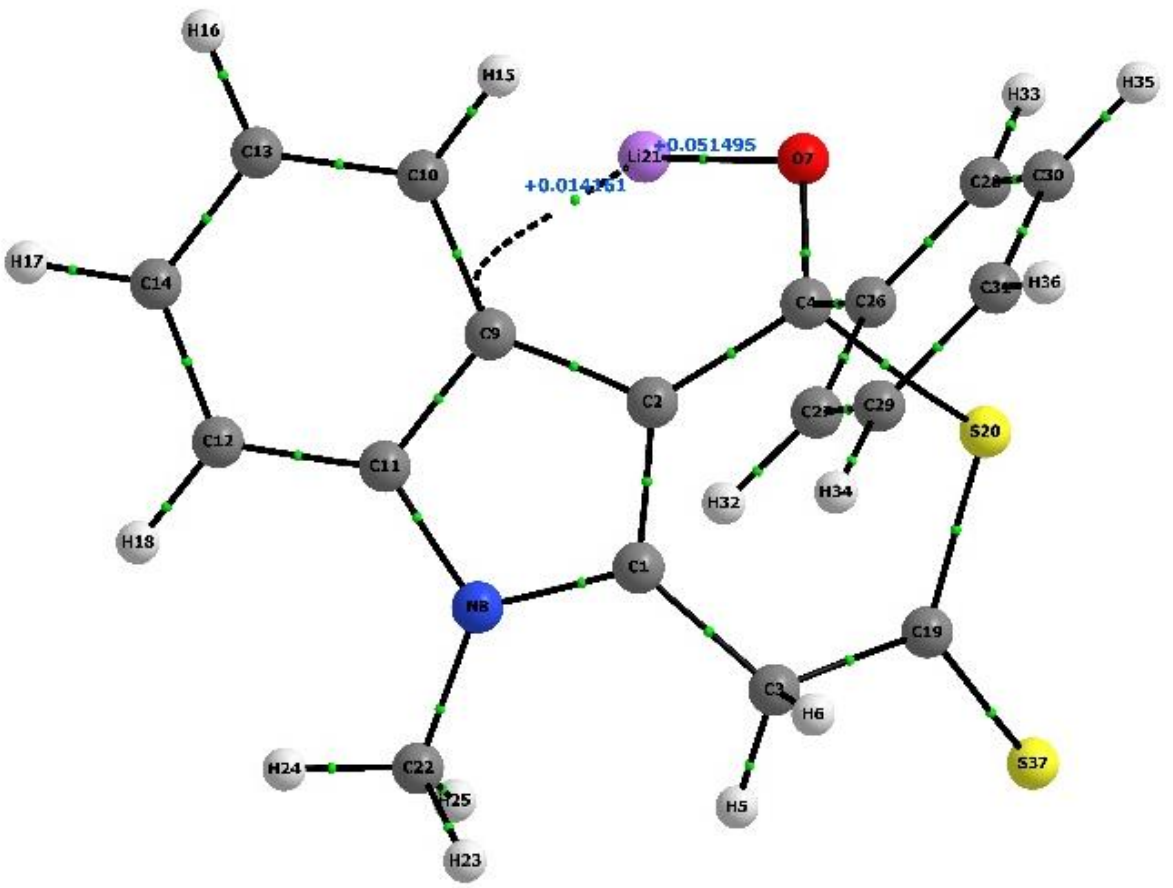

Figure S4. The attractive forces in system $\mathbf{T S 1}_{\mathbf{A}}$ according to AIM calculations. $\rho\left(r_{\mathrm{c}}\right)-$ electron density values.

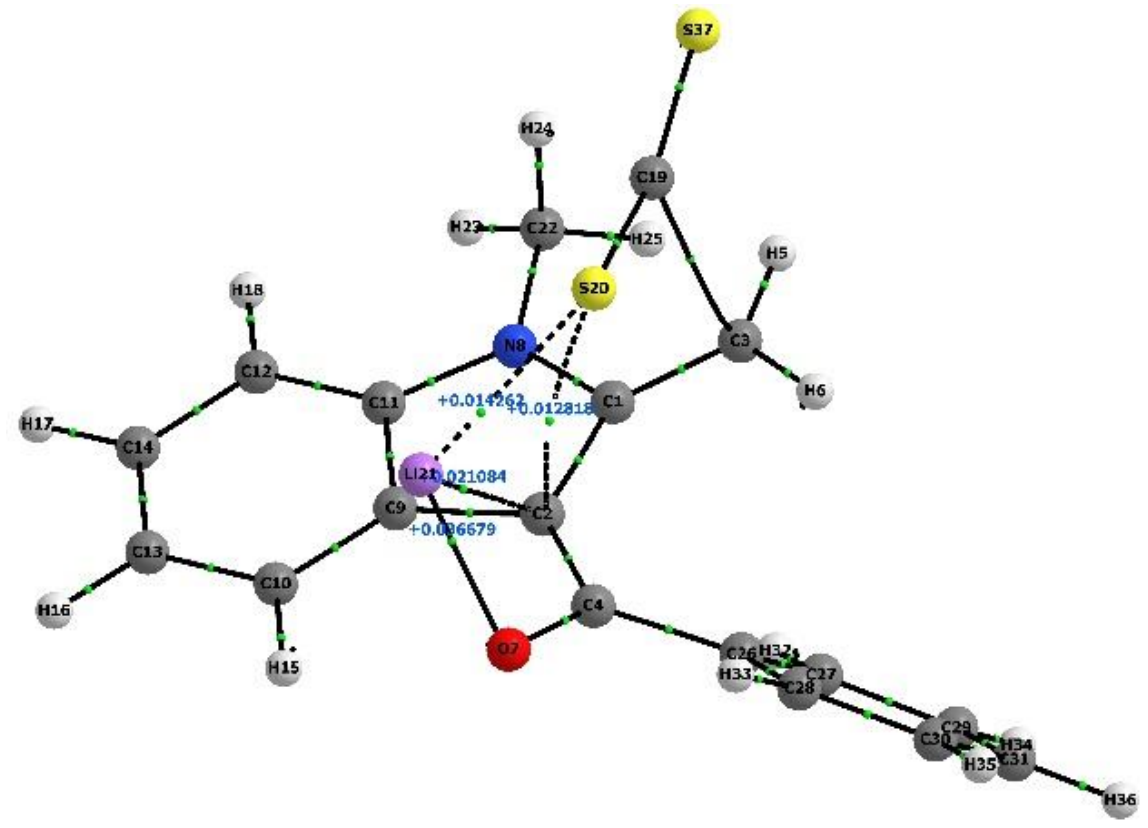


Figure S5. The attractive forces in system 25a according to AIM calculations. $\rho\left(r_{\mathrm{c}}\right)-$ electron density values.

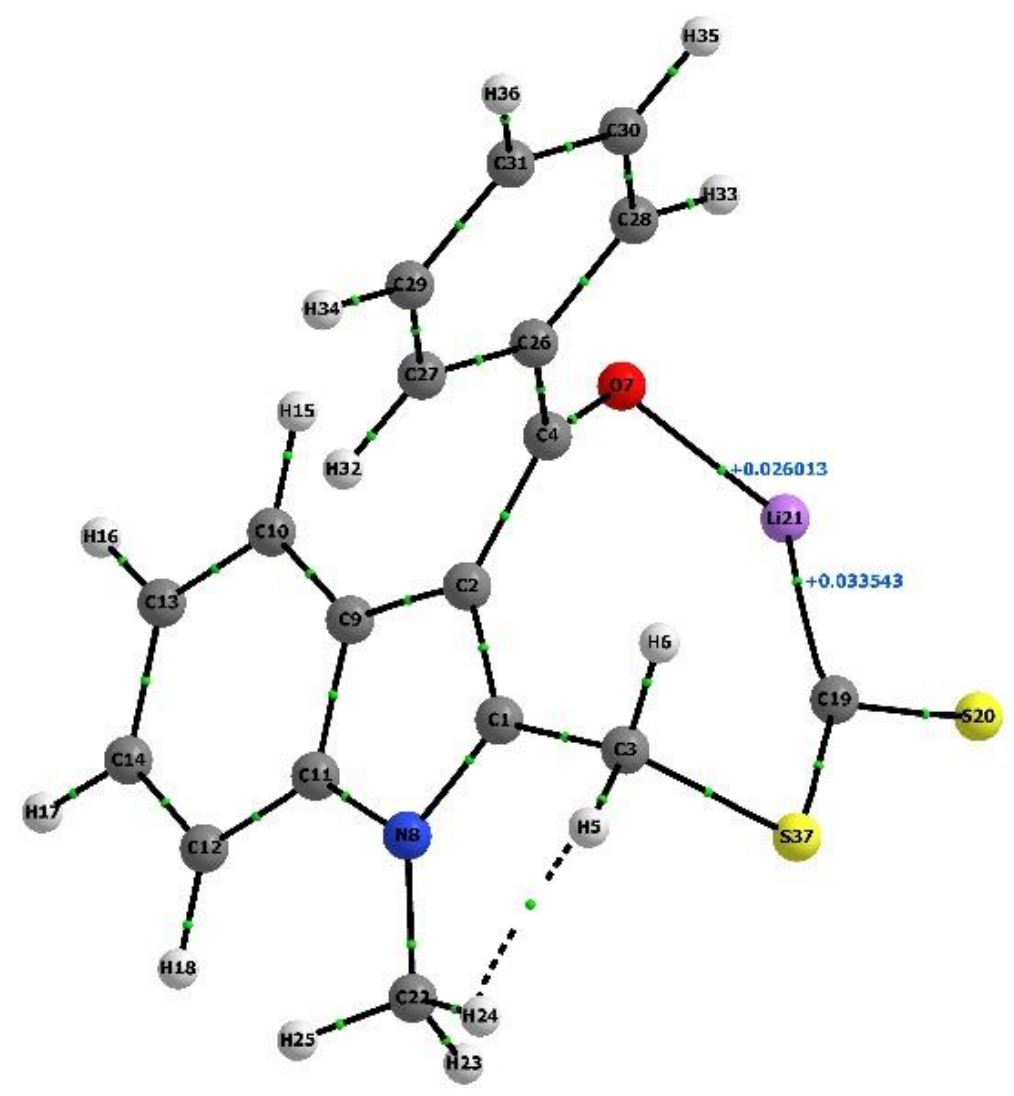

Figure S6. The attractive forces in system 21a according to AIM calculations. $\rho\left(r_{\mathrm{c}}\right)-$ electron density values.

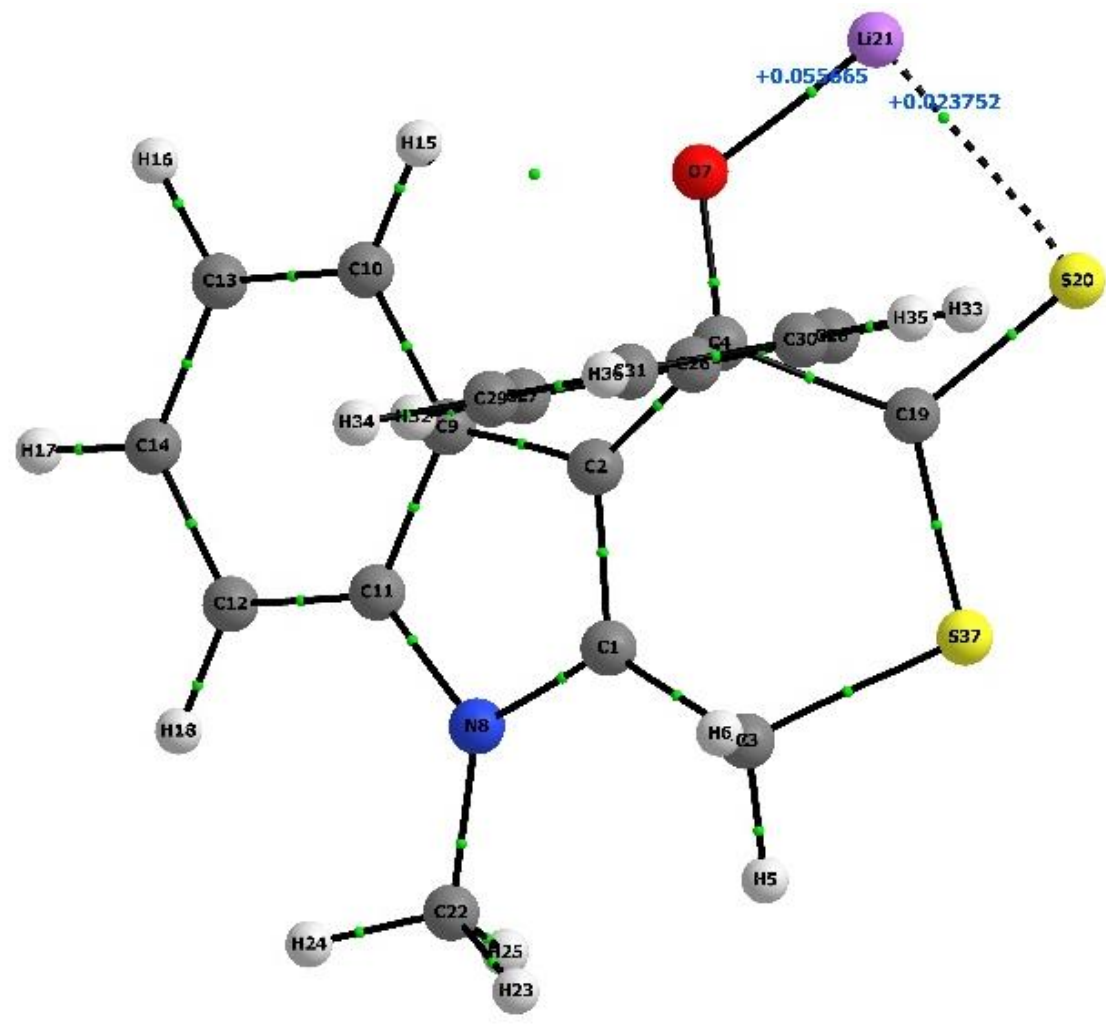


Figure S7. The attractive forces in system TS2 $2_{\mathbf{B}}$ according to AIM calculations. $\rho\left(r_{\mathrm{c}}\right)-$ electron density values.

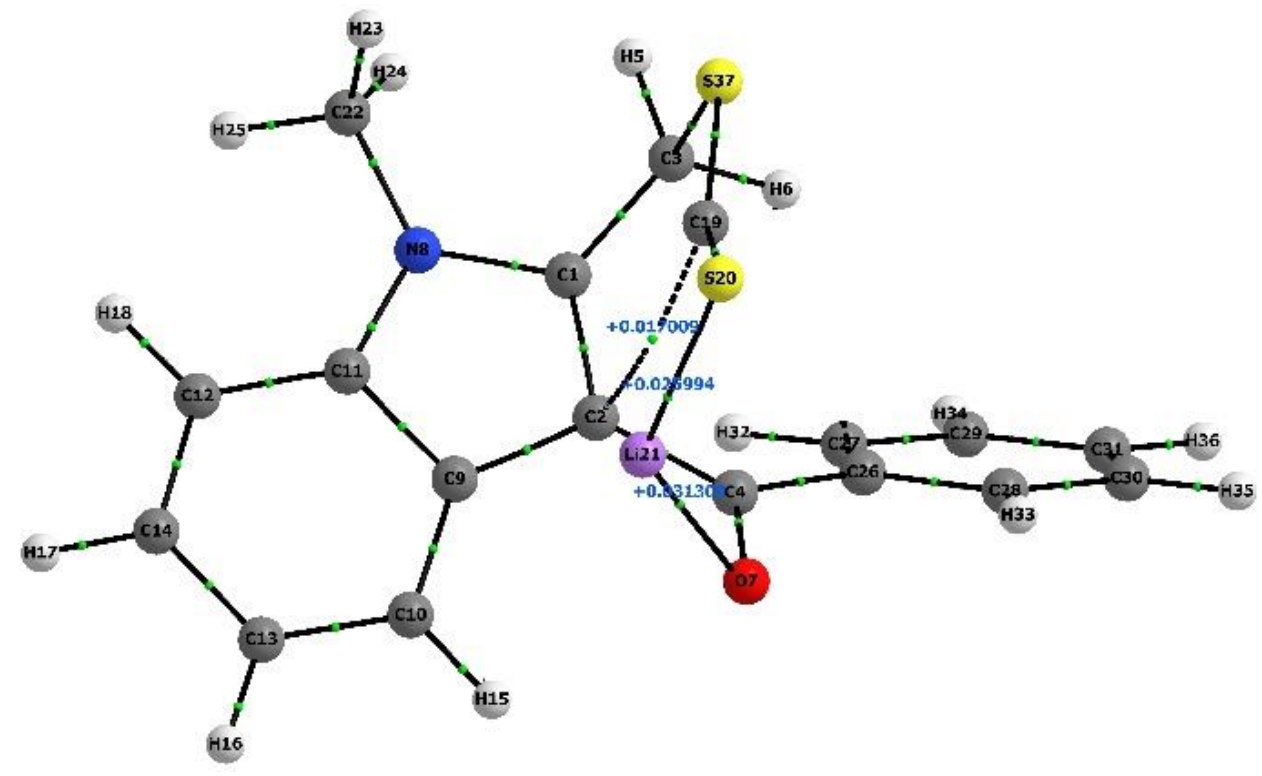


Figure S8. The structure 5a main geometric characteristics according to gas-phase calculations DFT B3LYP/6-311++G**. Results of $\mathrm{X}$-ray analysis are given in the parentheses. Bond lengths in $\AA$, bond angles in degrees.

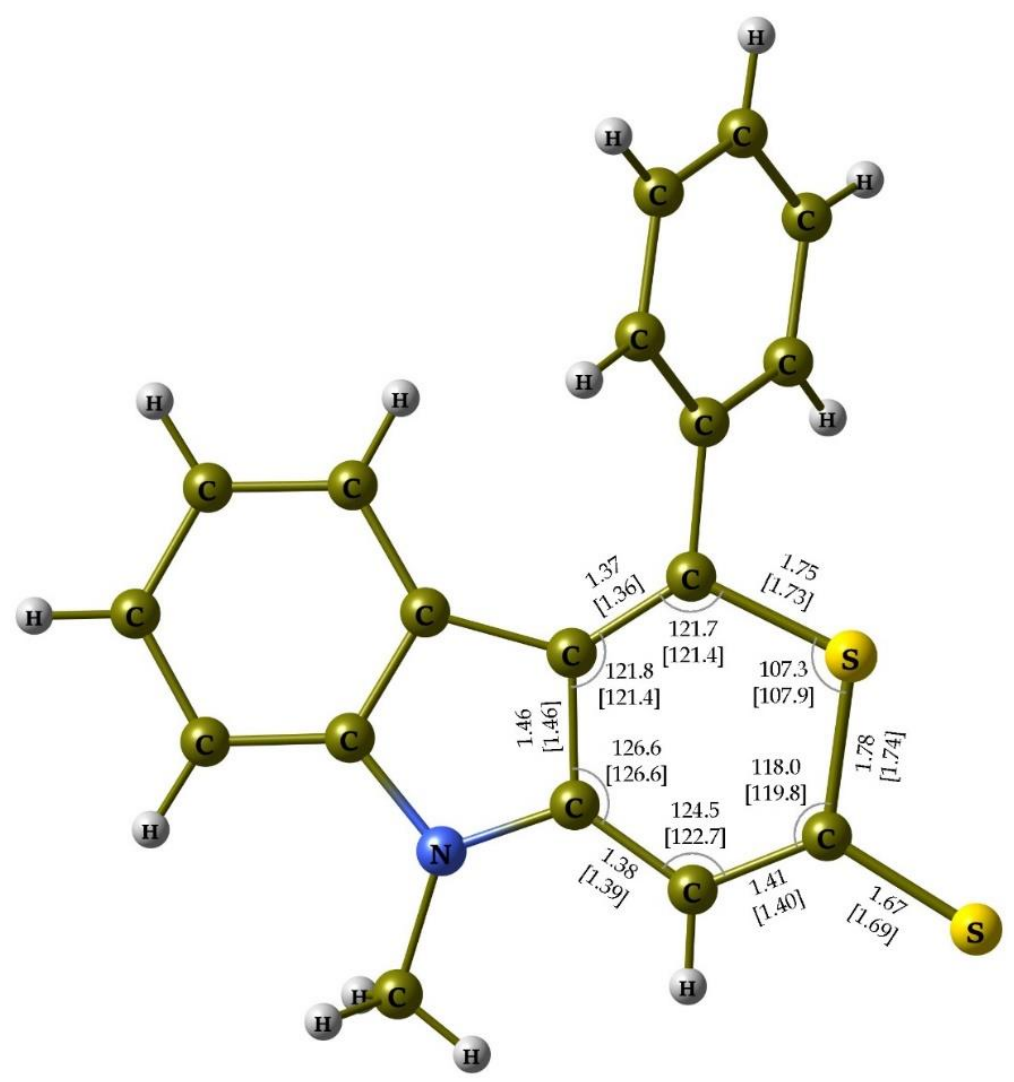

Table S1. Relative Gibbs energies $\Delta \mathrm{G}$ (in $\mathrm{kcal} / \mathrm{mol}$ ) of stationary points on the minimum energy paths for the reactions shown on Scheme 5.

\begin{tabular}{|c|c|c|c|}
\hline \multirow{2}{*}{ Structure } & \multicolumn{3}{|c|}{$\Delta \mathrm{G}$} \\
\hline & B3LYP (THF, $\left.-78^{\circ} \mathrm{C}\right)$ & PBE1PBE (gas phase) & MP2//HF (gas phase) \\
\hline $2 \mathrm{a}+\mathrm{CS}_{2}$ & 0.0 & 0.0 & 0.0 \\
\hline TS1a & 14.8 & 4.1 & $\begin{array}{l}-5.9 \\
\end{array}$ \\
\hline $24 a$ & -10.0 & -27.1 & -34.1 \\
\hline TS1b & - & -6.4 & -12.7 \\
\hline $23 a$ & - & -7.6 & -13.2 \\
\hline TS2a & - & - & 2.5 \\
\hline $25 a$ & - & - & 2.5 \\
\hline TS2b & 29.8 & 8.8 & 4.2 \\
\hline $21 \mathrm{a}$ & 13.4 & -7.4 & -17.3 \\
\hline
\end{tabular}


Table S2. Optimized geometries of stationary points of interaction paths of $\mathrm{CS}_{\mathbf{2}}$ with indole 2a. PCM/B3LYP/6-311++G(d,p) calculations (THF, $\left.-78{ }^{\circ} \mathrm{C}\right)$. Coordinates in Angstroms. Sum of electronic and thermal Free Energies in a.u. are in parentheses.

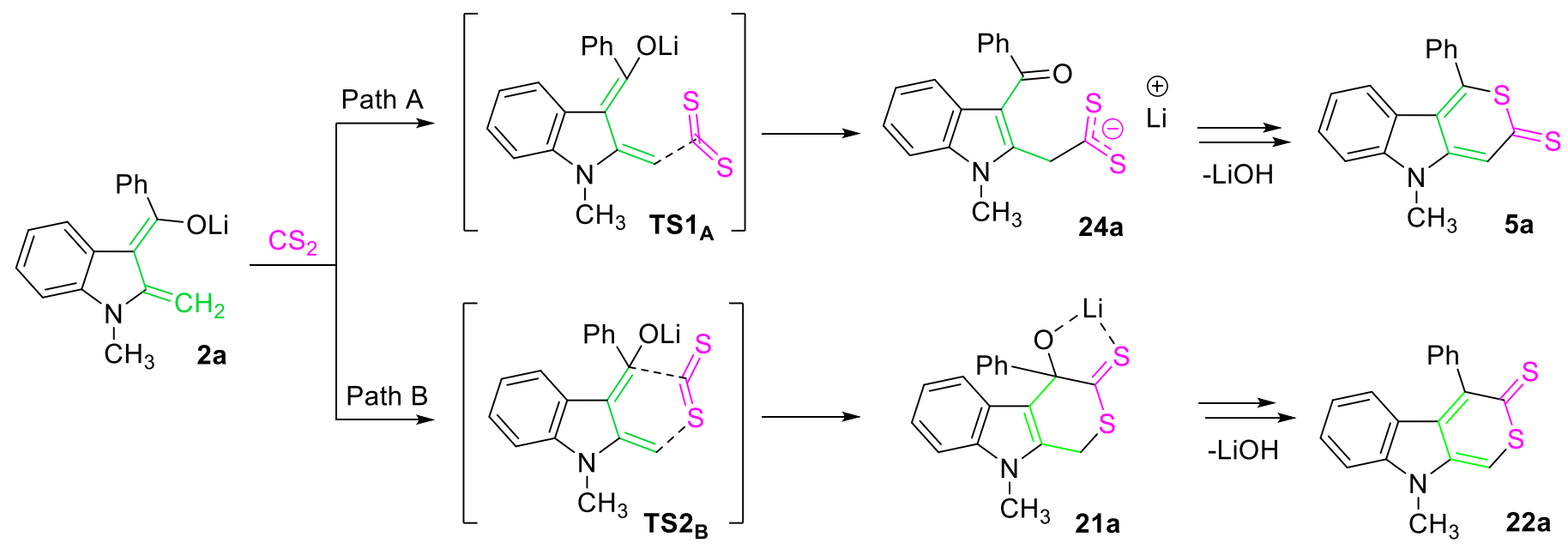

\begin{tabular}{|c|c|c|c|c|c|c|c|}
\hline \multicolumn{4}{|c|}{$\begin{array}{c}\mathbf{2 a} \\
v_{\text {im. }}=0 \\
0(-793,788)\end{array}$} & \multicolumn{4}{|c|}{$\begin{array}{c}\mathbf{C S}_{\mathbf{2}} \\
v_{\mathrm{im} .}=0(-834,55804)\end{array}$} \\
\hline C & 0.400929000 & 1.345748000 & -0.037051000 & $\mathrm{C}$ & -0.000004000 & 0.000258000 & -0.000006000 \\
\hline C & 0.344819000 & -0.133395000 & -0.016993000 & $S$ & -0.918582000 & -0.002797000 & -1.262071000 \\
\hline C & -0.568486000 & 2.298133000 & -0.088371000 & $S$ & 0.918583000 & 0.002700000 & 1.262073000 \\
\hline C & -0.764859000 & -0.963043000 & -0.012045000 & & & & \\
\hline $\mathrm{H}$ & -0.317612000 & 3.349887000 & -0.104511000 & & & & \\
\hline $\mathrm{H}$ & -1.612543000 & 2.035314000 & -0.113457000 & & & & \\
\hline 0 & -0.680975000 & -2.252245000 & -0.013261000 & & & & \\
\hline $\mathrm{N}$ & 1.768683000 & 1.680950000 & -0.004759000 & & & & \\
\hline C & 1.729251000 & -0.600278000 & -0.002571000 & & & & \\
\hline C & 2.318297000 & -1.865850000 & 0.008771000 & & & & \\
\hline C & 2.566837000 & 0.544577000 & 0.003783000 & & & & \\
\hline C & 3.954433000 & 0.438577000 & 0.018672000 & & & & \\
\hline C & 3.717036000 & -1.981587000 & 0.025081000 & & & & \\
\hline C & 4.522840000 & -0.844276000 & 0.029754000 & & & & \\
\hline $\mathrm{H}$ & 1.690145000 & -2.745157000 & 0.003498000 & & & & \\
\hline $\mathrm{H}$ & 4.173493000 & -2.965681000 & 0.032751000 & & & & \\
\hline $\mathrm{H}$ & 5.602896000 & -0.944238000 & 0.040840000 & & & & \\
\hline $\mathrm{H}$ & 4.592012000 & 1.314556000 & 0.020777000 & & & & \\
\hline $\mathrm{Li}$ & -1.435272000 & -3.885782000 & -0.011374000 & & & & \\
\hline C & 2.246848000 & 3.043648000 & -0.025189000 & & & & \\
\hline $\mathrm{H}$ & 1.838041000 & 3.612374000 & 0.816674000 & & & & \\
\hline $\mathrm{H}$ & 3.332157000 & 3.053620000 & 0.051080000 & & & & \\
\hline $\mathrm{H}$ & 1.959594000 & 3.551707000 & -0.953099000 & & & & \\
\hline C & -2.174255000 & -0.430765000 & 0.003022000 & & & & \\
\hline C & -2.817793000 & -0.159142000 & 1.215934000 & & & & \\
\hline C & -2.892600000 & -0.289549000 & -1.189982000 & & & & \\
\hline C & -4.151546000 & 0.248714000 & 1.236097000 & & & & \\
\hline C & -4.225518000 & 0.121410000 & -1.171756000 & & & & \\
\hline C & -4.858953000 & 0.391937000 & 0.041840000 & & & & \\
\hline $\mathrm{H}$ & -2.268305000 & -0.258633000 & 2.146064000 & & & & \\
\hline $\mathrm{H}$ & -2.402114000 & -0.491511000 & -2.136432000 & & & & \\
\hline $\mathrm{H}$ & -4.636592000 & 0.459218000 & 2.183193000 & & & & \\
\hline $\mathrm{H}$ & -4.767779000 & 0.234058000 & -2.104418000 & & & & \\
\hline $\mathrm{H}$ & -5.894257000 & 0.713980000 & 0.056572000 & & & & \\
\hline
\end{tabular}




\begin{tabular}{|c|c|c|c|c|c|c|c|}
\hline \multicolumn{4}{|c|}{$\begin{array}{c}\text { TS1 }_{\mathbf{A}} \\
V_{\text {im. }}=-318.9 \mathrm{~cm}^{-1}(-1628,32243)\end{array}$} & \multicolumn{4}{|c|}{$\begin{array}{c}\text { TS2 }_{\mathrm{B}} \\
V_{\text {im. }}=-276.1 \mathrm{~cm}^{-1}(-1628,29853)\end{array}$} \\
\hline C & -0.484186000 & 0.515990000 & -0.877918000 & C & 0.733162000 & -0.936222000 & 0.910397000 \\
\hline C & -0.679381000 & -0.721750000 & -0.140079000 & C & 0.585536000 & -0.290038000 & -0.340189000 \\
\hline C & 0.658775000 & 1.245466000 & -1.141255000 & C & -0.254124000 & -1.076384000 & 1.923580000 \\
\hline C & 0.295291000 & -1.594332000 & 0.378437000 & C & -0.630080000 & 0.191585000 & -0.958024000 \\
\hline $\mathrm{H}$ & 0.601550000 & 2.144615000 & -1.734729000 & $\mathrm{H}$ & 0.052407000 & -1.516907000 & 2.866262000 \\
\hline $\mathrm{H}$ & 1.629504000 & 0.791680000 & -1.040004000 & $\mathrm{H}$ & -1.240227000 & -1.387225000 & 1.611178000 \\
\hline 0 & 0.022202000 & -2.470006000 & 1.264392000 & 0 & -0.590442000 & 1.092702000 & -1.847501000 \\
\hline $\mathrm{N}$ & -1.752816000 & 1.030527000 & -1.147416000 & $\mathrm{~N}$ & 2.094333000 & -1.108392000 & 1.162125000 \\
\hline C & -2.115442000 & -0.879828000 & 0.037087000 & C & 1.921205000 & -0.073492000 & -0.852920000 \\
\hline C & -2.920253000 & -1.861349000 & 0.624105000 & C & 2.429801000 & 0.473367000 & -2.040805000 \\
\hline C & -2.740735000 & 0.215639000 & -0.610037000 & C & 2.824806000 & -0.607763000 & 0.102925000 \\
\hline C & -4.126209000 & 0.353507000 & -0.657670000 & C & 4.208600000 & -0.601237000 & -0.105144000 \\
\hline C & -4.312012000 & -1.733167000 & 0.576517000 & C & 3.804578000 & 0.480257000 & -2.251186000 \\
\hline C & -4.907318000 & -0.635389000 & -0.051580000 & C & 4.683799000 & -0.049819000 & -1.290397000 \\
\hline $\mathrm{H}$ & -2.466511000 & -2.718121000 & 1.101937000 & $\mathrm{H}$ & 1.752982000 & 0.883580000 & -2.778276000 \\
\hline $\mathrm{H}$ & -4.936057000 & -2.493770000 & 1.032876000 & $\mathrm{H}$ & 4.206453000 & 0.901554000 & -3.165869000 \\
\hline $\mathrm{H}$ & -5.987700000 & -0.545800000 & -0.074196000 & $\mathrm{H}$ & 5.752472000 & -0.026671000 & -1.472667000 \\
\hline $\mathrm{H}$ & -4.598213000 & 1.196359000 & -1.147467000 & $\mathrm{H}$ & 4.896614000 & -1.001780000 & 0.629320000 \\
\hline C & 1.178834000 & 2.539103000 & 0.902600000 & C & -0.921343000 & 1.705460000 & 1.131032000 \\
\hline$S$ & 0.520735000 & 1.739878000 & 2.117647000 & $S$ & -1.244377000 & 3.223238000 & 0.556580000 \\
\hline $\mathrm{Li}$ & -0.297836000 & -3.102942000 & 2.917714000 & $\mathrm{Li}$ & -0.697004000 & 2.920506000 & -1.863887000 \\
\hline C & -1.982859000 & 2.296562000 & -1.815064000 & C & 2.658920000 & -1.720679000 & 2.352720000 \\
\hline $\mathrm{H}$ & -3.047616000 & 2.422984000 & -1.997877000 & $\mathrm{H}$ & 2.037607000 & -2.558295000 & 2.671922000 \\
\hline $\mathrm{H}$ & -1.630458000 & 3.134212000 & -1.205114000 & $\mathrm{H}$ & 3.651098000 & -2.107421000 & 2.124080000 \\
\hline $\mathrm{H}$ & -1.464665000 & 2.322455000 & -2.776914000 & $\mathrm{H}$ & 2.742659000 & -1.005309000 & 3.176341000 \\
\hline C & 1.710232000 & -1.607583000 & -0.128360000 & C & -1.925330000 & -0.569454000 & -0.833536000 \\
\hline C & 1.975764000 & -1.834486000 & -1.484000000 & C & -1.943081000 & -1.969763000 & -0.812639000 \\
\hline C & 2.782945000 & -1.500100000 & 0.764584000 & C & -3.146203000 & 0.116630000 & -0.882037000 \\
\hline C & 3.288315000 & -1.950666000 & -1.938050000 & C & -3.151353000 & -2.666805000 & -0.817868000 \\
\hline C & 4.096121000 & -1.591387000 & 0.307669000 & C & -4.352446000 & -0.576310000 & -0.869021000 \\
\hline C & 4.352182000 & -1.821725000 & -1.044882000 & C & -4.360004000 & -1.972920000 & -0.837726000 \\
\hline $\mathrm{H}$ & 1.150499000 & -1.922969000 & -2.181333000 & $\mathrm{H}$ & -1.007721000 & -2.516810000 & -0.801805000 \\
\hline $\mathrm{H}$ & 2.585361000 & -1.323874000 & 1.815682000 & $\mathrm{H}$ & -3.139609000 & 1.198897000 & -0.912522000 \\
\hline $\mathrm{H}$ & 3.480244000 & -2.137712000 & -2.988819000 & $\mathrm{H}$ & -3.146336000 & -3.751208000 & -0.810489000 \\
\hline $\mathrm{H}$ & 4.919143000 & -1.484566000 & 1.005635000 & $\mathrm{H}$ & -5.288627000 & -0.029104000 & -0.885832000 \\
\hline $\mathrm{H}$ & 5.373675000 & -1.900549000 & -1.399814000 & $\mathrm{H}$ & -5.300014000 & -2.513318000 & -0.834639000 \\
\hline $\mathrm{S}$ & 2.039044000 & 3.745322000 & 0.291175000 & $\mathrm{~S}$ & -0.806510000 & 0.935959000 & 2.546221000 \\
\hline
\end{tabular}




\begin{tabular}{|c|c|c|c|c|c|c|c|}
\hline \multicolumn{4}{|c|}{$21 a$} & \multicolumn{4}{|c|}{$24 a$} \\
\hline \multicolumn{4}{|c|}{$v_{\text {im. }}=0(-1628,32465)$} & \multicolumn{4}{|c|}{$V_{\text {im. }}=0(-1628,36782)$} \\
\hline c & 0.868098000 & -0.446593000 & -1.194819000 & C & -0.468052000 & 0.344559000 & -0.476608000 \\
\hline c & 0.630402000 & -0.112342000 & 0.115674000 & C & -0.595687000 & -0.919653000 & 0.105900000 \\
\hline c & -0.183523000 & -0.905651000 & -2.136847000 & C & 0.735327000 & 1.220229000 & -0.654893000 \\
\hline c & -0.757083000 & -0.155031000 & 0.758560000 & C & 0.431880000 & -1.789397000 & 0.679132000 \\
\hline $\mathrm{H}$ & 0.204120000 & -1.430040000 & -3.009421000 & $\mathrm{H}$ & 0.842770000 & 1.518994000 & -1.700563000 \\
\hline $\mathrm{H}$ & -0.817228000 & -0.082900000 & -2.476294000 & $\mathrm{H}$ & 1.630472000 & 0.645479000 & -0.412864000 \\
\hline 0 & -0.725824000 & 0.000150000 & 2.098321000 & 0 & 0.158532000 & -2.567780000 & 1.596243000 \\
\hline $\mathrm{N}$ & 2.211304000 & -0.290247000 & -1.502984000 & $\mathrm{~N}$ & -1.712244000 & 0.797655000 & -0.846342000 \\
\hline c & 1.893983000 & 0.269395000 & 0.682122000 & C & -2.009691000 & -1.227718000 & 0.125163000 \\
\hline c & 2.319785000 & 0.716179000 & 1.946023000 & C & -2.768119000 & -2.321739000 & 0.568714000 \\
\hline c & 2.859302000 & 0.140151000 & -0.359658000 & C & -2.670703000 & -0.140575000 & -0.491272000 \\
\hline c & 4.213911000 & 0.428501000 & -0.159338000 & C & -4.055503000 & -0.109534000 & -0.673695000 \\
\hline c & 3.662945000 & 1.007543000 & 2.143007000 & C & -4.146458000 & -2.298143000 & 0.390780000 \\
\hline c & 4.602169000 & 0.863401000 & 1.102217000 & C & -4.784556000 & -1.204065000 & -0.222137000 \\
\hline $\mathrm{H}$ & 1.595047000 & 0.822561000 & 2.741451000 & $\mathrm{H}$ & -2.283717000 & -3.159550000 & 1.052543000 \\
\hline $\mathrm{H}$ & 3.999647000 & 1.351138000 & 3.115174000 & $\mathrm{H}$ & -4.744090000 & -3.134483000 & 0.735805000 \\
\hline $\mathrm{H}$ & 5.645461000 & 1.093799000 & 1.287414000 & $\mathrm{H}$ & -5.861962000 & -1.211783000 & -0.342081000 \\
\hline H & 4.941636000 & 0.316593000 & -0.954536000 & $\mathrm{H}$ & -4.549970000 & 0.732251000 & -1.143151000 \\
\hline c & -1.350584000 & -1.574224000 & 0.341208000 & C & 0.790331000 & 2.477119000 & 0.225120000 \\
\hline $\mathrm{s}$ & -2.077431000 & -2.575205000 & 1.454982000 & $\mathrm{~s}$ & 0.198614000 & 2.405167000 & 1.813912000 \\
\hline $\mathrm{Li}$ & -1.458610000 & -0.991098000 & 3.375498000 & $\mathrm{Li}$ & 1.053570000 & 4.764036000 & 1.831074000 \\
\hline c & 2.869306000 & -0.601612000 & -2.761861000 & C & -2.036218000 & 2.088727000 & -1.437832000 \\
\hline $\mathrm{H}$ & 2.150066000 & -0.562167000 & -3.578038000 & $\mathrm{H}$ & -2.757395000 & 1.947030000 & -2.244071000 \\
\hline $\mathrm{H}$ & 3.642213000 & 0.141110000 & -2.963511000 & $\mathrm{H}$ & -2.462226000 & 2.762755000 & -0.690166000 \\
\hline $\mathrm{H}$ & 3.329245000 & -1.594299000 & -2.740766000 & $\mathrm{H}$ & -1.142123000 & 2.545291000 & -1.853965000 \\
\hline c & -1.700313000 & 0.928568000 & 0.133647000 & C & 1.827263000 & -1.806789000 & 0.120839000 \\
\hline c & -1.172156000 & 2.130961000 & -0.348148000 & C & 2.063422000 & -1.780372000 & -1.259563000 \\
\hline c & -3.093349000 & 0.787054000 & 0.171010000 & C & 2.909872000 & -1.956454000 & 0.997768000 \\
\hline c & -2.007880000 & 3.164203000 & -0.774601000 & C & 3.361347000 & -1.899210000 & -1.754055000 \\
\hline c & -3.931704000 & 1.813043000 & -0.262880000 & C & 4.207750000 & -2.047124000 & 0.504165000 \\
\hline c & -3.392742000 & 3.009076000 & -0.737886000 & C & 4.435542000 & -2.023416000 & -0.873567000 \\
\hline $\mathrm{H}$ & -0.097522000 & 2.265496000 & -0.384306000 & $\mathrm{H}$ & 1.229666000 & -1.684889000 & -1.945547000 \\
\hline $\mathrm{H}$ & -3.534159000 & -0.128501000 & 0.548179000 & $\mathrm{H}$ & 2.723241000 & -1.994103000 & 2.064376000 \\
\hline $\mathrm{H}$ & -1.573701000 & 4.090077000 & -1.136946000 & $\mathrm{H}$ & 3.533047000 & -1.895166000 & -2.824449000 \\
\hline H & -5.007519000 & 1.676736000 & -0.230213000 & $\mathrm{H}$ & 5.041438000 & -2.143188000 & 1.190570000 \\
\hline $\mathrm{H}$ & -4.043701000 & 3.808268000 & -1.074656000 & $\mathrm{H}$ & 5.445921000 & -2.105311000 & -1.258176000 \\
\hline $\mathrm{s}$ & -1.279676000 & -2.146031000 & -1.297501000 & $\mathrm{~s}$ & 1.522959000 & 3.857617000 & -0.452086000 \\
\hline \multicolumn{4}{|c|}{$22 a$} & \multicolumn{4}{|c|}{$5 a$} \\
\hline \multicolumn{4}{|c|}{ Vim. $=0(-1544,88508)$} & \multicolumn{4}{|c|}{ Vim. $=-0(-1544.89802)$} \\
\hline c & -1.586268000 & 1.362402000 & -0.005954000 & C & -1.755046000 & 0.327451000 & 0.056394000 \\
\hline c & -0.580094000 & 0.313947000 & -0.002601000 & C & -0.300529000 & 0.311989000 & 0.016178000 \\
\hline c & -1.310645000 & 2.694409000 & 0.002122000 & C & -2.586735000 & -0.782750000 & 0.055327000 \\
\hline c & 0.791085000 & 0.557367000 & -0.000952000 & C & 0.411623000 & -0.854333000 & 0.011912000 \\
\hline $\mathrm{H}$ & -2.069692000 & 3.466020000 & 0.005924000 & $\mathrm{H}$ & -3.661019000 & -0.649104000 & 0.060438000 \\
\hline $\mathrm{N}$ & -2.846044000 & 0.785449000 & -0.015180000 & $\mathrm{~N}$ & -2.171676000 & 1.628891000 & 0.069278000 \\
\hline c & -1.327720000 & -0.932130000 & -0.000733000 & C & 0.118035000 & 1.711067000 & -0.045960000 \\
\hline c & -0.960714000 & -2.287734000 & 0.004043000 & C & 1.352772000 & 2.355683000 & -0.151135000 \\
\hline c & -2.707922000 & -0.590019000 & -0.003518000 & C & -1.064756000 & 2.482981000 & -0.016139000 \\
\hline c & -3.706414000 & -1.568311000 & 0.003586000 & C & -1.041359000 & 3.873305000 & -0.080814000 \\
\hline c & -1.950882000 & -3.260076000 & 0.008299000 & C & 1.385985000 & 3.747523000 & -0.215839000 \\
\hline c & -3.308709000 & -2.899329000 & 0.008745000 & C & 0.203143000 & 4.494939000 & -0.178152000 \\
\hline H & 0.080003000 & -2.576446000 & 0.005249000 & $\mathrm{H}$ & 2.273058000 & 1.789150000 & -0.185204000 \\
\hline $\mathrm{H}$ & -1.675067000 & -4.307583000 & 0.012584000 & $\mathrm{H}$ & 2.338977000 & 4.256175000 & -0.299076000 \\
\hline $\mathrm{H}$ & -4.066026000 & -3.675062000 & 0.014178000 & $\mathrm{H}$ & 0.250411000 & 5.576401000 & -0.231329000 \\
\hline $\mathrm{H}$ & -4.757062000 & -1.308687000 & 0.006747000 & $\mathrm{H}$ & -1.949867000 & 4.461383000 & -0.067333000 \\
\hline c & 1.354155000 & 1.872097000 & 0.001139000 & C & -2.126828000 & -2.106481000 & 0.023331000 \\
\hline S & 3.012519000 & 2.239770000 & 0.000869000 & $\mathrm{~s}$ & -0.383436000 & -2.409629000 & 0.022671000 \\
\hline c & -4.086429000 & 1.541210000 & -0.003752000 & C & -3.562395000 & 2.060094000 & 0.083273000 \\
\hline $\mathrm{H}$ & -4.102664000 & 2.255904000 & -0.830112000 & $\mathrm{H}$ & -3.614269000 & 3.090751000 & 0.426863000 \\
\hline $\mathrm{H}$ & -4.925498000 & 0.861139000 & -0.126677000 & $\mathrm{H}$ & -4.006681000 & 1.991740000 & -0.913386000 \\
\hline H & -4.208309000 & 2.080209000 & 0.940133000 & $\mathrm{H}$ & -4.134651000 & 1.443529000 & 0.776243000 \\
\hline c & 1.745157000 & -0.596307000 & -0.001539000 & C & 1.893281000 & -0.959421000 & 0.024832000 \\
\hline c & 2.183214000 & -1.150268000 & -1.208423000 & C & 2.613801000 & -0.563884000 & 1.160345000 \\
\hline c & 2.189380000 & -1.147454000 & 1.204387000 & C & 2.579796000 & -1.489575000 & -1.075435000 \\
\hline
\end{tabular}




\begin{tabular}{|c|c|c|c|c|c|c|c|}
\hline c & 3.047556000 & -2.243883000 & -1.209334000 & c & 4.000360000 & -0.686325000 & 1.187134000 \\
\hline C & 3.053621000 & -2.241131000 & 1.203339000 & c & 3.968661000 & -1.601246000 & -1.046870000 \\
\hline c & 3.483314000 & -2.793288000 & -0.003470000 & c & 4.680740000 & -1.201435000 & 0.082978000 \\
\hline $\mathrm{H}$ & 1.846509000 & -0.724858000 & -2.147199000 & $\mathrm{H}$ & 2.085532000 & -0.168214000 & 2.020004000 \\
\hline $\mathrm{H}$ & 1.857609000 & -0.719817000 & 2.143899000 & $\mathrm{H}$ & 2.028421000 & -1.800812000 & -1.955111000 \\
\hline $\mathrm{H}$ & 3.380163000 & -2.665567000 & -2.151228000 & $\mathrm{H}$ & 4.548529000 & -0.382921000 & 2.071601000 \\
\hline $\mathrm{H}$ & 3.390931000 & -2.660764000 & 2.144475000 & H & 4.491791000 & -2.002803000 & -1.906955000 \\
\hline $\mathrm{H}$ & 4.155558000 & -3.643898000 & -0.004210000 & $\mathrm{H}$ & 5.760410000 & -1.294426000 & 0.105578000 \\
\hline $\mathrm{s}$ & 0.305340000 & 3.280997000 & 0.006643000 & $\mathrm{~S}$ & -3.106078000 & -3.492283000 & -0.006137000 \\
\hline \multicolumn{4}{|c|}{ LiOH } & & & & \\
\hline \multicolumn{4}{|c|}{$V_{\text {im. }}=0(-83.46076)$} & & & & \\
\hline 0 & 0.000000000 & 0.000000000 & 0.346599000 & & & & \\
\hline $\mathrm{H}$ & 0.000000000 & 0.000000000 & 1.301361000 & & & & \\
\hline $\mathrm{Li}$ & 0.000000000 & 0.000000000 & -1.358051000 & & & & \\
\hline
\end{tabular}

Table S3. Optimized geometries of stationary points of interaction paths of $\mathrm{CS}_{2}$ with indole 2a. PBE1PBE/6-311++G** calculations (gas phase). Coordinates in Angstroms. Sum of electronic and thermal Free Energies in a.u. are in parentheses.

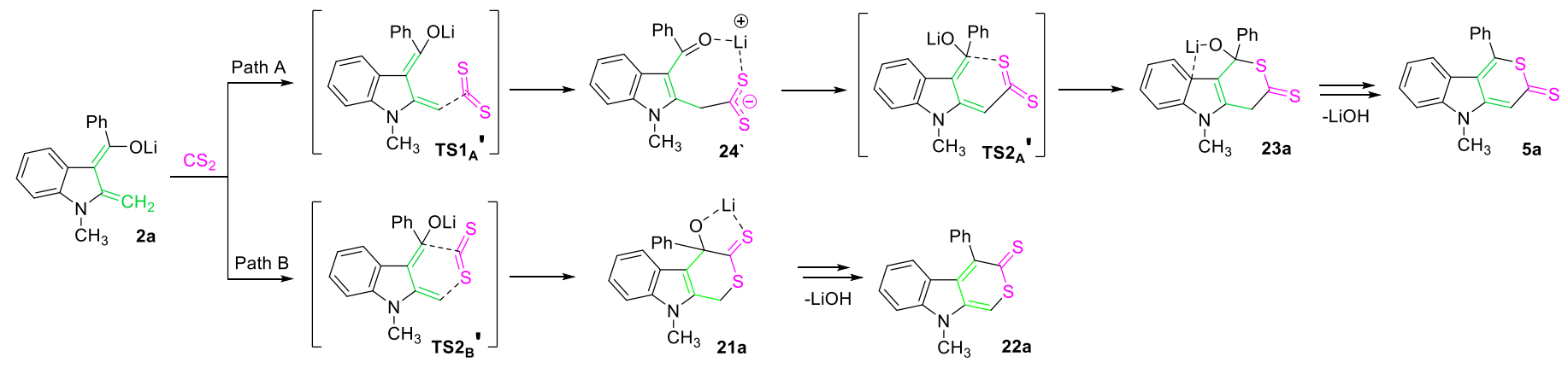

\begin{tabular}{|c|c|c|c|c|c|c|c|}
\hline \multicolumn{4}{|c|}{$\begin{array}{c}\mathbf{2 a} \\
v_{\text {im. }}=0(-792,79442)\end{array}$} & \multicolumn{4}{|c|}{$\begin{array}{c}\mathbf{2 4 a} \\
V_{\text {im. }}=0 \\
0(-1627,04214)\end{array}$} \\
\hline C & 0.387835000000 & 1.335273000000 & -0.184552000000 & $\mathrm{C}$ & -0.458795000000 & 0.342975000000 & -0.466748000000 \\
\hline C & 0.331183000000 & -0.137801000000 & -0.079623000000 & $\mathrm{C}$ & -0.581874000000 & -0.924067000000 & 0.088270000000 \\
\hline C & -0.569155000000 & 2.260333000000 & -0.414886000000 & $\mathrm{C}$ & 0.733654000000 & 1.223529000000 & -0.623400000000 \\
\hline C & -0.772846000000 & -0.947830000000 & -0.050749000000 & C & 0.444024000000 & -1.797387000000 & 0.661532000000 \\
\hline $\mathrm{H}$ & -0.321913000000 & 3.310962000000 & -0.497347000000 & $\mathrm{H}$ & 0.876198000000 & 1.526642000000 & -1.666021000000 \\
\hline $\mathrm{H}$ & -1.604124000000 & 1.981641000000 & -0.533862000000 & $\mathrm{H}$ & 1.630935000000 & 0.658381000000 & -0.353918000000 \\
\hline 0 & -0.694759000000 & -2.247238000000 & -0.048995000000 & 0 & 0.170951000000 & -2.581744000000 & 1.556828000000 \\
\hline $\mathrm{N}$ & 1.739875000000 & 1.675809000000 & -0.051739000000 & $\mathrm{~N}$ & -1.699509000000 & 0.796673000000 & -0.835076000000 \\
\hline C & 1.712938000000 & -0.596977000000 & -0.008966000000 & $\mathrm{C}$ & -1.986631000000 & -1.238518000000 & 0.092433000000 \\
\hline C & 2.308290000000 & -1.850728000000 & 0.058387000000 & C & -2.733775000000 & -2.340406000000 & 0.520514000000 \\
\hline C & 2.536736000000 & 0.550035000000 & 0.007790000000 & C & -2.648563000000 & -0.148217000000 & -0.504256000000 \\
\hline C & 3.919207000000 & 0.455665000000 & 0.082075000000 & C & -4.029346000000 & -0.120897000000 & -0.689173000000 \\
\hline C & 3.699711000000 & -1.957244000000 & 0.137114000000 & C & -4.106484000000 & -2.320166000000 & 0.339586000000 \\
\hline C & 4.492036000000 & -0.816478000000 & 0.147872000000 & C & -4.747685000000 & -1.224369000000 & -0.257675000000 \\
\hline $\mathrm{H}$ & 1.687930000000 & -2.736839000000 & 0.040357000000 & $\mathrm{H}$ & -2.238559000000 & -3.176084000000 & 0.999974000000 \\
\hline $\mathrm{H}$ & 4.164173000000 & -2.937072000000 & 0.184553000000 & $\mathrm{H}$ & -4.701850000000 & -3.163952000000 & 0.672784000000 \\
\hline $\mathrm{H}$ & 5.572224000000 & -0.908322000000 & 0.203371000000 & $\mathrm{H}$ & -5.825854000000 & -1.237981000000 & -0.379610000000 \\
\hline $\mathrm{H}$ & 4.550489000000 & 1.337558000000 & 0.085484000000 & $\mathrm{H}$ & -4.529195000000 & 0.727158000000 & -1.144948000000 \\
\hline $\mathrm{Li}$ & -0.976772000000 & -3.854609000000 & 0.054640000000 & $\mathrm{C}$ & 0.752742000000 & 2.468326000000 & 0.249810000000 \\
\hline C & 2.212179000000 & 3.022935000000 & -0.156020000000 & $\mathrm{~s}$ & 0.029311000000 & 2.400136000000 & 1.779074000000 \\
\hline $\mathrm{H}$ & 1.675406000000 & 3.669540000000 & 0.546351000000 & $\mathrm{Li}$ & 1.008106000000 & 4.458854000000 & 1.732033000000 \\
\hline $\mathrm{H}$ & 3.272794000000 & 3.060563000000 & 0.091269000000 & $\mathrm{C}$ & -2.023691000000 & 2.096321000000 & -1.370200000000 \\
\hline $\mathrm{H}$ & 2.072744000000 & 3.425132000000 & -1.168756000000 & $\mathrm{H}$ & -2.712436000000 & 1.986108000000 & -2.211734000000 \\
\hline C & -2.171539000000 & -0.429251000000 & 0.013503000000 & $\mathrm{H}$ & -2.488908000000 & 2.727969000000 & -0.606475000000 \\
\hline C & -2.647000000000 & 0.193005000000 & 1.168309000000 & $\mathrm{H}$ & -1.122851000000 & 2.592946000000 & -1.727925000000 \\
\hline C & -3.051036000000 & -0.645643000000 & -1.048506000000 & $\mathrm{C}$ & 1.839410000000 & -1.784525000000 & 0.115432000000 \\
\hline
\end{tabular}




\begin{tabular}{|c|c|c|c|c|c|c|c|}
\hline C & -3.976133000000 & 0.588229000000 & 1.260517000000 & C & 2.092621000000 & -1.670625000000 & -1.252677000000 \\
\hline C & -4.377235000000 & -0.237182000000 & -0.963262000000 & C & 2.903966000000 & -1.992302000000 & 0.994386000000 \\
\hline C & -4.844287000000 & 0.377613000000 & 0.193924000000 & $\mathrm{C}$ & 3.394262000000 & -1.752349000000 & -1.733814000000 \\
\hline $\mathrm{H}$ & -1.961066000000 & 0.376223000000 & 1.989275000000 & $\mathrm{C}$ & 4.205288000000 & -2.046898000000 & 0.516199000000 \\
\hline $\mathrm{H}$ & -2.683534000000 & -1.121072000000 & -1.953173000000 & C & 4.452266000000 & -1.929483000000 & -0.849213000000 \\
\hline $\mathrm{H}$ & -4.334272000000 & 1.068684000000 & 2.165755000000 & $\mathrm{H}$ & 1.262629000000 & -1.537269000000 & -1.939808000000 \\
\hline $\mathrm{H}$ & -5.046932000000 & -0.394682000000 & -1.803275000000 & $\mathrm{H}$ & 2.689147000000 & -2.112171000000 & 2.050944000000 \\
\hline \multirow[t]{4}{*}{$\mathrm{H}$} & -5.879959000000 & 0.695039000000 & 0.262657000000 & $\mathrm{H}$ & 3.582691000000 & -1.680650000000 & -2.800177000000 \\
\hline & & & & $\mathrm{H}$ & 5.030283000000 & -2.191252000000 & 1.206435000000 \\
\hline & & & & $\mathrm{H}$ & 5.469524000000 & -1.983465000000 & -1.223769000000 \\
\hline & & & & $\mathrm{S}$ & 1.569098000000 & 3.819780000000 & -0.383260000000 \\
\hline \multicolumn{4}{|c|}{$\begin{array}{c}\mathbf{T S 1}_{\mathbf{A}}^{\prime} \\
11.5 \mathrm{~cm}^{-1}(-1626,99476)\end{array}$} & \multicolumn{4}{|c|}{$\begin{array}{c}\text { TS2 } \\
\mathbf{A} \\
.0 \mathrm{~cm}^{-1}(-1627,01135)\end{array}$} \\
\hline C & -0.437874000000 & 0.318409000000 & -1.029595000000 & C & 0.798018000000 & 0.864387000000 & 0.635797000000 \\
\hline C & -0.201684000000 & -0.795158000000 & -0.130977000000 & C & 0.525460000000 & -0.254245000000 & -0.116009000000 \\
\hline C & 0.355285000000 & 1.397284000000 & -1.302327000000 & C & -0.151205000000 & 1.958604000000 & 0.963188000000 \\
\hline C & 0.982861000000 & -1.192137000000 & 0.552803000000 & C & -0.753222000000 & -0.603756000000 & -0.827723000000 \\
\hline $\mathrm{H}$ & 0.020332000000 & 2.187244000000 & -1.959644000000 & $\mathrm{H}$ & 0.338661000000 & 2.800426000000 & 1.451978000000 \\
\hline $\mathrm{H}$ & 1.399017000000 & 1.389343000000 & -1.024074000000 & $\mathrm{H}$ & -0.925052000000 & 1.585182000000 & 1.648382000000 \\
\hline 0 & 0.876343000000 & -1.756119000000 & 1.684043000000 & 0 & -0.566776000000 & -1.188044000000 & -1.979712000000 \\
\hline $\mathrm{N}$ & -1.774421000000 & 0.239869000000 & -1.421276000000 & $\mathrm{~N}$ & 2.127578000000 & 0.881577000000 & 0.994692000000 \\
\hline C & -1.495622000000 & -1.414430000000 & 0.107207000000 & $\mathrm{C}$ & 1.761781000000 & -0.975256000000 & -0.238399000000 \\
\hline C & -1.927459000000 & -2.479517000000 & 0.906178000000 & C & 2.138148000000 & -2.186051000000 & -0.853742000000 \\
\hline C & -2.434574000000 & -0.740519000000 & -0.712708000000 & C & 2.747126000000 & -0.220219000000 & 0.455166000000 \\
\hline C & -3.773404000000 & -1.100975000000 & -0.733121000000 & C & 4.077514000000 & -0.629454000000 & 0.510881000000 \\
\hline C & -3.276803000000 & -2.840941000000 & 0.884807000000 & $\mathrm{C}$ & 3.467261000000 & -2.579835000000 & -0.803830000000 \\
\hline C & -4.183412000000 & -2.159452000000 & 0.080266000000 & C & 4.425502000000 & -1.807290000000 & -0.132991000000 \\
\hline $\mathrm{H}$ & -1.213237000000 & -3.037137000000 & 1.504058000000 & $\mathrm{H}$ & 1.383186000000 & -2.822025000000 & -1.309990000000 \\
\hline $\mathrm{H}$ & -3.617043000000 & -3.666904000000 & 1.500138000000 & $\mathrm{H}$ & 3.766050000000 & -3.513821000000 & -1.267954000000 \\
\hline $\mathrm{H}$ & -5.228818000000 & -2.449536000000 & 0.082774000000 & $\mathrm{H}$ & 5.456762000000 & -2.141929000000 & -0.105071000000 \\
\hline $\mathrm{H}$ & -4.492358000000 & -0.577036000000 & -1.353115000000 & $\mathrm{H}$ & 4.830439000000 & -0.051866000000 & 1.036199000000 \\
\hline C & -0.263729000000 & 2.759718000000 & 0.890807000000 & $\mathrm{C}$ & -0.881669000000 & 2.485856000000 & -0.266498000000 \\
\hline $\mathrm{S}$ & 0.194361000000 & 1.764152000000 & 2.046546000000 & $\mathrm{~S}$ & -1.491216000000 & 1.322606000000 & -1.358994000000 \\
\hline $\mathrm{Li}$ & -0.588128000000 & -0.644914000000 & 2.021154000000 & $\mathrm{Li}$ & 1.077809000000 & -0.782639000000 & -2.476575000000 \\
\hline C & -2.418011000000 & 1.199228000000 & -2.276870000000 & $C$ & 2.784265000000 & 1.937410000000 & 1.732344000000 \\
\hline $\mathrm{H}$ & -3.320656000000 & 0.757415000000 & -2.701415000000 & $\mathrm{H}$ & 2.239684000000 & 2.158063000000 & 2.652537000000 \\
\hline $\mathrm{H}$ & -2.689006000000 & 2.115626000000 & -1.739581000000 & $\mathrm{H}$ & 3.786119000000 & 1.609624000000 & 2.006034000000 \\
\hline $\mathrm{H}$ & -1.751471000000 & 1.460614000000 & -3.101610000000 & $\mathrm{H}$ & 2.863768000000 & 2.851454000000 & 1.136506000000 \\
\hline C & 2.343664000000 & -0.985860000000 & -0.002561000000 & C & -1.873431000000 & -1.166657000000 & 0.014121000000 \\
\hline C & 2.587204000000 & -1.040237000000 & -1.376454000000 & $\mathrm{C}$ & -1.823851000000 & -1.202144000000 & 1.405951000000 \\
\hline C & 3.415836000000 & -0.827846000000 & 0.877785000000 & $\mathrm{C}$ & -2.982483000000 & -1.708096000000 & -0.638480000000 \\
\hline C & 3.881918000000 & -0.921381000000 & -1.861971000000 & $\mathrm{C}$ & -2.875721000000 & -1.746703000000 & 2.136500000000 \\
\hline C & 4.708202000000 & -0.692586000000 & 0.390639000000 & $\mathrm{C}$ & -4.031931000000 & -2.247490000000 & 0.089484000000 \\
\hline C & 4.943152000000 & -0.739281000000 & -0.980091000000 & $\mathrm{C}$ & -3.985179000000 & -2.264987000000 & 1.481580000000 \\
\hline $\mathrm{H}$ & 1.756802000000 & -1.185914000000 & -2.058918000000 & $\mathrm{H}$ & -0.947450000000 & -0.825097000000 & 1.923991000000 \\
\hline $\mathrm{H}$ & 3.216735000000 & -0.817799000000 & 1.943719000000 & $\mathrm{H}$ & -2.998445000000 & -1.703238000000 & -1.722797000000 \\
\hline $\mathrm{H}$ & 4.065676000000 & -0.975495000000 & -2.930082000000 & $\mathrm{H}$ & -2.821736000000 & -1.773815000000 & 3.220403000000 \\
\hline $\mathrm{H}$ & 5.535675000000 & -0.557668000000 & 1.079666000000 & $\mathrm{H}$ & -4.891242000000 & -2.660998000000 & -0.429194000000 \\
\hline $\mathrm{H}$ & 5.954723000000 & -0.641640000000 & -1.361353000000 & $\mathrm{H}$ & -4.806273000000 & -2.689177000000 & 2.050507000000 \\
\hline S & -0.775304000000 & 4.100696000000 & 0.255308000000 & $\mathrm{~S}$ & -1.075349000000 & 4.110770000000 & -0.470443000000 \\
\hline
\end{tabular}




\section{TS1'} $V_{\text {im. }}=-256.5(-1626,98718)$

C $\quad 0.415401000000 \quad-0.274134000000$

C $0.288886000000-0.619250000000$

C $-0.484000000000 \quad 0.512524000000$

C $-0.871766000000-0.560388000000$

H -0.257150000000

H -1.538682000000

O -0.710316000000

N 1.742028000000

C 1.606079000000

C 2.107245000000

C 2.477027000000

C 3.829901000000

C 3.457260000000

C 4.305823000000

H 1.446251000000

H 3.859989000000

H 5.358372000000

H 4.501662000000

C 0.076409000000

S $\quad 0.403305000000$

Li 0.580905000000

C 2.283748000000

H 2.379591000000

H 1.643353000000

H $\quad 3.268555000000$

C -2.242158000000

C -2.477971000000

C -3.322062000000

C -3.778902000000

C -4.619374000000

C -4.849223000000

H -1.639041000000

H -3.123128000000

H -3.959086000000

H -5.454848000000

H -5.865340000000

S -0.283855000000

$-1.624209000000$

2.532796000000
1.274494000000 $-0.087364000000$ 2.040270000000 3.094526000000 1.850369000000 $-2.165522000000$ 1.631362000000 $-0.552776000000$ $-1.773946000000$ 0.552994000000 0.459232000000 $-1.868902000000$ $-0.765843000000$ $-2.625184000000$ $-2.810326000000$ $-0.867246000000$ 1.304015000000 $-0.198327000000$ $-1.542475000000$ $-1.817163000000$ 2.921984000000 3.040726000000 3.716049000000 3.031519000000 $-0.453007000000$ 0.579815000000 $-1.097175000000$ 0.970473000000 $-0.690893000000$ 0.341902000000 1.061254000000 $-1.911349000000$ 1.762819000000 $-1.182375000000$ 0.653235000000 1.356827000000 $-0.951520000000$

24a`

$v_{\text {im. }}=0(-1627,04435)$

C $-0.516585000000 \quad 0.356102000000$

C $-0.126051000000-0.806169000000$

C $\quad 0.190095000000 \quad 1.660597000000$

C $1.116367000000-1.021011000000$

H $\quad 0.182975000000 \quad 2.083300000000$

H $1.238512000000 \quad 1.511132000000$

O $1.020031000000-1.283930000000$

N $\quad-1.812717000000 \quad 0.223830000000$

C $-1.279330000000-1.670986000000$

C $-1.526270000000-2.946025000000$

C $-2.302969000000-0.998212000000$

C $-3.563659000000-1.559960000000$

C $-2.780988000000-3.502453000000$

C $-3.787136000000-2.817802000000$

H $\quad-0.753095000000-3.481049000000$

H $\quad-2.993100000000-4.485470000000$

H $\quad-4.760183000000-3.281514000000$

H $\quad-4.345268000000-1.034376000000$

C $-0.400913000000 \quad 2.700984000000$

S $-0.827767000000 \quad 2.197037000000$

Li $-0.502821000000 \quad-0.078103000000$

C -2.637447000000

$23 a$

$$
v_{\text {im. }}=0(-1627,01335)
$$

C $0.815666000000 \quad 0.950941000000$

C $0.535593000000-0.175780000000$

C $-0.132133000000 \quad 2.040665000000$

C $-0.749771000000-0.540990000000$

H $\quad 0.376395000000 \quad 2.920204000000$

H -0.826798000000

O -0.558276000000

N 2.147148000000

1.693545000000

$-1.102237000000$

0.964954000000

C 1.765937000000

C 2.131651000000

C 2.758234000000

C 4.086607000000

C 3.458417000000

C 4.424727000000

H 1.369803000000

H 3.748414000000

H 5.454030000000

H 4.845205000000

C -0.993511000000

S -1.595237000000

Li 1.097968000000

C 2.816099000000

H 2.234193000000

H $\quad 3.783098000000$

H 2.975031000000

C -1.801769000000

C -1.743733000000

$-0.908249000000$

$-2.136224000000$

$-0.147827000000$

$-0.563019000000$

$-2.538676000000$

$-1.756912000000$

$-2.780109000000$

$-3.486130000000$

$-2.097296000000$

0.021998000000

2.491559000000

1.291688000000

$-0.825004000000$

2.024717000000

2.317039000000

1.663147000000

2.903728000000

$-1.210068000000$

$-1.218481000000$
0.585332000000

$-0.148513000000$

0.933992000000

$-0.843977000000$

1.329013000000

1.712605000000

$-2.016990000000$

0.944066000000

$-0.257971000000$

$-0.844855000000$

0.419011000000

0.476302000000

$-0.791122000000$

$-0.143290000000$

$-1.278999000000$

$-1.233018000000$

$-0.112661000000$

0.985058000000

$-0.235780000000$

$-1.293232000000$

$-2.519575000000$

1.663867000000

2.011789000000

1.031567000000

0.016622000000

1.408721000000
2.539593000000
H -3.175889000000

H -3.351267000000

H -2.014647000000

C 2.434124000000

C 2.560236000000

C 3.577921000000

C 3.816202000000

C 4.828667000000

C 4.947758000000

H 1.672047000000

H 3.459942000000

H 3.914583000000

H $\quad 5.714770000000$

H 5.929210000000

S -0.546077000000

-0.078103000000
1.261441000000

0.860446000000

1.638523000000

2.093069000000

$-0.917049000000$

$-0.935614000000$

$-0.822568000000$

$-0.851712000000$

$-0.733717000000$

$-0.747307000000$

$-1.031233000000$

$-0.813082000000$

$-0.871276000000$

$-0.650112000000$

$-0.677709000000$

4.252155000000

21a

$V_{\text {im. }}=-0(-1627,01302)$

C 0.827335000000

$-0.367872000000$

C 0.623433000000

C -0.261042000000

C -0.744638000000

H 0.084811000000

H -0.909327000000

O -0.694312000000

N 2.159487000000

C 1.895507000000

C 2.340709000000

C 2.832410000000

C 4.189350000000

C 3.685215000000

C 4.600473000000

H 1.621783000000

H 4.044776000000

H 5.649775000000

H 4.903137000000

C -1.342670000000

S -2.061815000000

Li -1.490663000000

C 2.786970000000

H 2.044360000000

H 3.529823000000

H $\quad 3.282597000000$

C -1.672530000000

C -1.129911000000

$-0.122100000000$

$-0.743574000000$

$-0.191368000000$

$-1.185632000000$

0.109299000000

$-0.105694000000$

$-0.207148000000$

0.208628000000

0.570559000000

0.139013000000

0.401009000000

0.837813000000

0.750591000000

0.633556000000

1.119282000000

0.960593000000

0.332442000000

$-1.566563000000$

$-2.633440000000$

$-1.211100000000$

$-0.440558000000$

$-0.392734000000$

0.336948000000

$-1.417439000000$

0.920298000000

2.165690000000

$-0.623601000000$

0.033930000000

$-0.683135000000$

0.782694000000

$-1.690384000000$

$-0.408737000000$

1.992611000000

$-1.027237000000$

0.087092000000

0.610442000000

$-0.614785000000$

$-0.803356000000$

0.430620000000

$-0.267759000000$

1.151872000000

0.837333000000

$-0.389174000000$ 


\begin{tabular}{|c|c|c|c|c|c|c|c|}
\hline C & -2.856503000000 & -1.864969000000 & -0.620629000000 & C & -3.055741000000 & 0.757400000000 & 0.131364000000 \\
\hline C & -2.733478000000 & -1.851685000000 & 2.153609000000 & C & -1.948175000000 & 3.221599000000 & -0.491743000000 \\
\hline C & -3.845735000000 & -2.492984000000 & 0.122002000000 & C & -3.875726000000 & 1.810019000000 & -0.261211000000 \\
\hline C & -3.790630000000 & -2.485889000000 & 1.513304000000 & C & -3.325383000000 & 3.047023000000 & -0.575268000000 \\
\hline $\mathrm{H}$ & -0.908208000000 & -0.746715000000 & 1.916730000000 & $\mathrm{H}$ & -0.055952000000 & 2.308575000000 & -0.041805000000 \\
\hline $\mathrm{H}$ & -2.876081000000 & -1.877813000000 & -1.704784000000 & $\mathrm{H}$ & -3.505023000000 & -0.198624000000 & 0.382691000000 \\
\hline $\mathrm{H}$ & -2.672528000000 & -1.856059000000 & 3.237512000000 & $\mathrm{H}$ & -1.506652000000 & 4.185438000000 & -0.726415000000 \\
\hline $\mathrm{H}$ & -4.663394000000 & -2.995443000000 & -0.385510000000 & $\mathrm{H}$ & -4.949397000000 & 1.660426000000 & -0.322729000000 \\
\hline $\mathrm{H}$ & -4.563433000000 & -2.979677000000 & 2.093651000000 & $\mathrm{H}$ & -3.964540000000 & 3.869073000000 & -0.881426000000 \\
\hline$S$ & -1.342729000000 & 4.092127000000 & -0.425276000000 & $S$ & -1.307786000000 & -2.040183000000 & -1.368071000000 \\
\hline \multicolumn{4}{|c|}{$22 a$} & \multicolumn{4}{|c|}{$5 a$} \\
\hline \multicolumn{4}{|c|}{$v_{\text {im. }}=0(-1543.69643)$} & \multicolumn{4}{|c|}{$V_{\text {im. }}=0(-1543.70754)$} \\
\hline C & 1.575841000000 & 1.365251000000 & 0.006249000000 & C & -1.749704000000 & 0.311413000000 & 0.067454000000 \\
\hline C & 0.573369000000 & 0.319620000000 & 0.003804000000 & $\mathrm{C}$ & -0.301048000000 & 0.307683000000 & 0.017556000000 \\
\hline C & 1.299225000000 & 2.691954000000 & -0.004191000000 & $\mathrm{C}$ & -2.571681000000 & -0.790897000000 & 0.067141000000 \\
\hline C & -0.785486000000 & 0.559444000000 & 0.001940000000 & $\mathrm{C}$ & 0.410883000000 & -0.855361000000 & 0.015441000000 \\
\hline $\mathrm{H}$ & 2.062917000000 & 3.461468000000 & -0.011448000000 & $\mathrm{H}$ & -3.649423000000 & -0.672254000000 & 0.074930000000 \\
\hline $\mathrm{N}$ & 2.830127000000 & 0.784544000000 & 0.016431000000 & $\mathrm{~N}$ & -2.170340000000 & 1.613675000000 & 0.086045000000 \\
\hline C & 1.318944000000 & -0.927156000000 & 0.001644000000 & C & 0.107331000000 & 1.703935000000 & -0.057147000000 \\
\hline C & 0.947318000000 & -2.273966000000 & -0.003469000000 & C & 1.331799000000 & 2.353415000000 & -0.188126000000 \\
\hline C & 2.692183000000 & -0.587697000000 & 0.003839000000 & C & -1.076845000000 & 2.466720000000 & -0.016402000000 \\
\hline C & 3.685643000000 & -1.562870000000 & -0.004743000000 & $\mathrm{C}$ & -1.060883000000 & 3.852962000000 & -0.089103000000 \\
\hline C & 1.934245000000 & -3.247578000000 & -0.008752000000 & $\mathrm{C}$ & 1.356733000000 & 3.741491000000 & -0.261652000000 \\
\hline C & 3.285790000000 & -2.891401000000 & -0.010178000000 & C & 0.175000000000 & 4.479659000000 & -0.208517000000 \\
\hline $\mathrm{H}$ & -0.097730000000 & -2.555774000000 & -0.004293000000 & $\mathrm{H}$ & 2.254383000000 & 1.788412000000 & -0.237791000000 \\
\hline $\mathrm{H}$ & 1.654882000000 & -4.295119000000 & -0.013504000000 & $\mathrm{H}$ & 2.306135000000 & 4.255044000000 & -0.365869000000 \\
\hline $\mathrm{H}$ & 4.042929000000 & -3.668967000000 & -0.016983000000 & $\mathrm{H}$ & 0.215168000000 & 5.562154000000 & -0.269190000000 \\
\hline $\mathrm{H}$ & 4.738664000000 & -1.305948000000 & -0.009881000000 & $\mathrm{H}$ & -1.973698000000 & 4.437140000000 & -0.067850000000 \\
\hline C & -1.361780000000 & 1.875620000000 & -0.000267000000 & $\mathrm{C}$ & -2.109241000000 & -2.122816000000 & 0.024046000000 \\
\hline S & -2.993683000000 & 2.206408000000 & 0.001382000000 & $S$ & -0.366538000000 & -2.404817000000 & 0.032361000000 \\
\hline C & 4.056362000000 & 1.532607000000 & 0.005218000000 & $\mathrm{C}$ & -3.551087000000 & 2.023099000000 & 0.107755000000 \\
\hline $\mathrm{H}$ & 4.076425000000 & 2.250079000000 & 0.832331000000 & $\mathrm{H}$ & -3.613942000000 & 3.065163000000 & 0.419286000000 \\
\hline $\mathrm{H}$ & 4.898619000000 & 0.853732000000 & 0.128968000000 & $\mathrm{H}$ & -4.018754000000 & 1.917720000000 & -0.877333000000 \\
\hline $\mathrm{H}$ & 4.184498000000 & 2.075547000000 & -0.938441000000 & $\mathrm{H}$ & -4.106820000000 & 1.421760000000 & 0.830544000000 \\
\hline C & -1.734477000000 & -0.586438000000 & 0.001999000000 & C & 1.885987000000 & -0.947473000000 & 0.029954000000 \\
\hline C & -2.165390000000 & -1.141825000000 & 1.205552000000 & C & 2.605406000000 & -0.456048000000 & 1.122869000000 \\
\hline C & -2.173712000000 & -1.135573000000 & -1.201439000000 & $\mathrm{C}$ & 2.571005000000 & -1.563006000000 & -1.020079000000 \\
\hline C & -3.013798000000 & -2.242252000000 & 1.205169000000 & C & 3.988704000000 & -0.566049000000 & 1.155173000000 \\
\hline C & -3.021930000000 & -2.236101000000 & -1.200877000000 & C & 3.956507000000 & -1.662468000000 & -0.987522000000 \\
\hline C & -3.440899000000 & -2.794067000000 & 0.002176000000 & C & 4.667386000000 & -1.165457000000 & 0.098516000000 \\
\hline $\mathrm{H}$ & -1.834544000000 & -0.707245000000 & 2.143324000000 & $\mathrm{H}$ & 2.072276000000 & 0.005396000000 & 1.947780000000 \\
\hline $\mathrm{H}$ & -1.849539000000 & -0.695989000000 & -2.139202000000 & $\mathrm{H}$ & 2.014567000000 & -1.952373000000 & -1.866402000000 \\
\hline $\mathrm{H}$ & -3.344581000000 & -2.667447000000 & 2.147281000000 & $\mathrm{H}$ & 4.538085000000 & -0.188195000000 & 2.011200000000 \\
\hline $\mathrm{H}$ & -3.359038000000 & -2.656556000000 & -2.142874000000 & $\mathrm{H}$ & 4.480436000000 & -2.133200000000 & -1.812723000000 \\
\hline $\mathrm{H}$ & -4.104619000000 & -3.652682000000 & 0.002224000000 & $\mathrm{H}$ & 5.748751000000 & -1.250227000000 & 0.125409000000 \\
\hline $\mathrm{S}$ & -0.313097000000 & 3.279070000000 & -0.008543000000 & $\mathrm{~S}$ & -3.064230000000 & -3.483750000000 & -0.018514000000 \\
\hline \multicolumn{4}{|c|}{ LiOH } & \multicolumn{4}{|c|}{$\mathrm{CS}_{2}$} \\
\hline \multicolumn{4}{|c|}{$V_{\text {im. }}=0(-83.30884)$} & \multicolumn{4}{|c|}{ Vim. $=0(-834,20679)$} \\
\hline $\mathrm{O}$ & 0.000000000000 & 0.000000000000 & 0.318403000000 & C & 0.000000000000 & 0.000000000000 & -0.000001000000 \\
\hline $\mathrm{H}$ & 0.000000000000 & 0.000000000000 & 1.267368000000 & s & 0.000000000000 & 0.000000000000 & -1.554790000000 \\
\hline $\mathrm{Li}$ & 0.000000000000 & 0.000000000000 & -1.271529000000 & S & 0.000000000000 & 0.000000000000 & 1.554790000000 \\
\hline
\end{tabular}


Table S4. Optimized geometries of stationary points of interaction paths of $\mathrm{CS}_{2}$ with indole 2a. MP2//HF/6-311++G** calculations (gas phase). Coordinates in Angstroms. Sum of electronic (MP2/6-311++G** single point) and thermal Free Energies (HF/6$\left.311++G^{* *}\right)$ in a.u. are in parentheses.

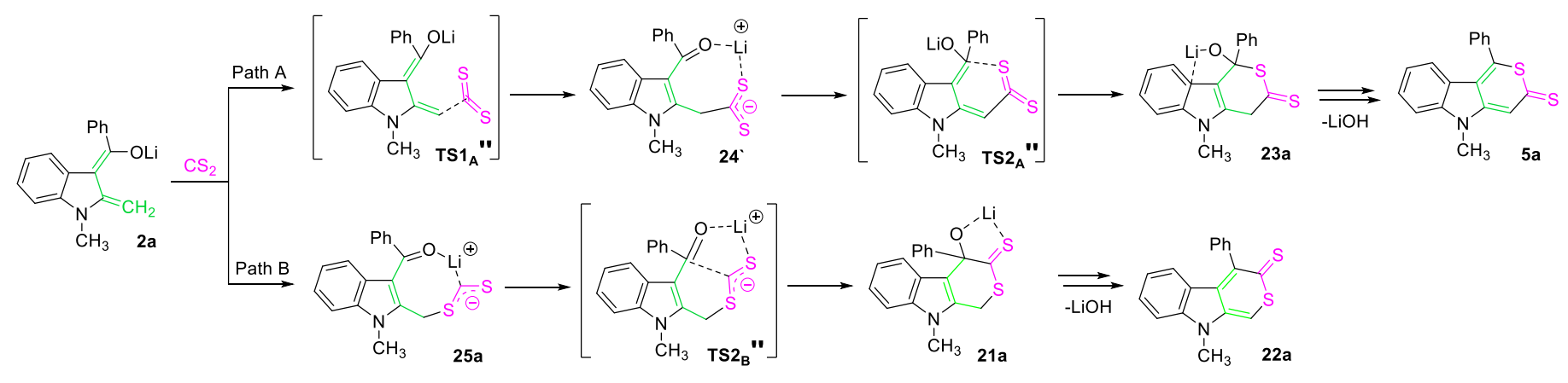

\begin{tabular}{|c|c|c|c|c|c|c|c|}
\hline \multicolumn{4}{|c|}{$2 a$} & \multicolumn{4}{|c|}{$5 a$} \\
\hline \multicolumn{4}{|c|}{$V_{\text {im. }}=0(-791,36428)$} & \multicolumn{4}{|c|}{$V_{\text {im. }}=0(-1541,56059)$} \\
\hline C & 0.403562000000 & 1.348346000000 & -0.163282000000 & C & -1.718359000000 & 0.470773000000 & 0.018378000000 \\
\hline c & 0.325236000000 & -0.136645000000 & -0.082175000000 & c & -0.269635000000 & 0.313503000000 & 0.010343000000 \\
\hline c & -0.530802000000 & 2.266628000000 & -0.431014000000 & c & -2.639886000000 & -0.536406000000 & 0.005498000000 \\
\hline c & -0.766330000000 & -0.931465000000 & -0.030110000000 & c & 0.310522000000 & -0.894735000000 & 0.008123000000 \\
\hline $\mathrm{H}$ & -0.288240000000 & 3.310201000000 & -0.483343000000 & $\mathrm{H}$ & -3.688922000000 & -0.316411000000 & -0.003795000000 \\
\hline $\mathrm{H}$ & -1.549128000000 & 1.994569000000 & -0.604217000000 & $\mathrm{~N}$ & -2.000331000000 & 1.799479000000 & 0.033925000000 \\
\hline 0 & -0.699642000000 & -2.222101000000 & 0.004285000000 & c & 0.278281000000 & 1.677704000000 & -0.003909000000 \\
\hline $\mathrm{N}$ & 1.748697000000 & 1.670772000000 & 0.049396000000 & c & 1.562045000000 & 2.195194000000 & -0.029672000000 \\
\hline c & 1.719752000000 & -0.604529000000 & -0.025573000000 & c & -0.818229000000 & 2.542491000000 & 0.003039000000 \\
\hline c & 2.304999000000 & -1.853620000000 & -0.024047000000 & c & -0.659816000000 & 3.915547000000 & -0.020304000000 \\
\hline c & 2.539212000000 & 0.532846000000 & 0.043877000000 & c & 1.733893000000 & 3.571204000000 & -0.049209000000 \\
\hline c & 3.913598000000 & 0.432066000000 & 0.104955000000 & C & 0.633718000000 & 4.416041000000 & -0.045299000000 \\
\hline c & 3.695582000000 & -1.970048000000 & 0.041923000000 & $\mathrm{H}$ & 2.417246000000 & 1.548291000000 & -0.036414000000 \\
\hline c & 4.484533000000 & -0.840931000000 & 0.104648000000 & $\mathrm{H}$ & 2.725737000000 & 3.984067000000 & -0.070142000000 \\
\hline $\mathrm{H}$ & 1.694079000000 & -2.730599000000 & -0.084508000000 & $\mathrm{H}$ & 0.781470000000 & 5.481031000000 & -0.064038000000 \\
\hline $\mathrm{H}$ & 4.150800000000 & -2.944816000000 & 0.036805000000 & $\mathrm{H}$ & -1.497470000000 & 4.586285000000 & -0.023814000000 \\
\hline $\mathrm{H}$ & 5.555515000000 & -0.935058000000 & 0.149384000000 & C & -2.298751000000 & -1.906635000000 & -0.002122000000 \\
\hline $\mathrm{H}$ & 4.543928000000 & 1.300282000000 & 0.148059000000 & $\mathrm{~s}$ & -0.610765000000 & -2.370770000000 & 0.006989000000 \\
\hline $\mathrm{Li}$ & -1.165216000000 & -3.780189000000 & 0.189680000000 & c & -3.341558000000 & 2.340243000000 & 0.029081000000 \\
\hline c & 2.254498000000 & 3.008521000000 & -0.076979000000 & $\mathrm{H}$ & -3.302273000000 & 3.398888000000 & 0.232309000000 \\
\hline $\mathrm{H}$ & 1.662867000000 & 3.687543000000 & 0.525386000000 & $\mathrm{H}$ & -3.823132000000 & 2.184704000000 & -0.931198000000 \\
\hline $\mathrm{H}$ & 3.271519000000 & 3.049368000000 & 0.285576000000 & $\mathrm{H}$ & -3.935307000000 & 1.870607000000 & 0.803607000000 \\
\hline $\mathrm{H}$ & 2.236531000000 & 3.355948000000 & -1.109600000000 & C & 1.786858000000 & -1.124133000000 & 0.008992000000 \\
\hline c & -2.177032000000 & -0.415125000000 & 0.018651000000 & C & 2.483098000000 & -1.176316000000 & 1.209532000000 \\
\hline c & -2.705664000000 & 0.097533000000 & 1.195281000000 & C & 2.466352000000 & -1.306200000000 & -1.188247000000 \\
\hline c & -3.000889000000 & -0.535718000000 & -1.094236000000 & C & 3.851248000000 & -1.391063000000 & 1.210370000000 \\
\hline C & -4.034660000000 & 0.486133000000 & 1.258438000000 & C & 3.835042000000 & -1.519256000000 & -1.184808000000 \\
\hline c & -4.326910000000 & -0.138269000000 & -1.035581000000 & C & 4.529007000000 & -1.560016000000 & 0.013596000000 \\
\hline C & -4.848687000000 & 0.371862000000 & 0.143159000000 & $\mathrm{H}$ & 1.956747000000 & -1.047108000000 & 2.138200000000 \\
\hline $\mathrm{H}$ & -2.070976000000 & 0.207007000000 & 2.056354000000 & $\mathrm{H}$ & 1.927479000000 & -1.277621000000 & -2.118281000000 \\
\hline $\mathrm{H}$ & -2.596661000000 & -0.923600000000 & -2.013020000000 & $\mathrm{H}$ & 4.384383000000 & -1.429155000000 & 2.143225000000 \\
\hline $\mathrm{H}$ & -4.430898000000 & 0.885250000000 & 2.175605000000 & $\mathrm{H}$ & 4.355504000000 & -1.656144000000 & -2.115531000000 \\
\hline $\mathrm{H}$ & -4.949769000000 & -0.221500000000 & -1.909021000000 & $\mathrm{H}$ & 5.591008000000 & -1.728343000000 & 0.015379000000 \\
\hline $\mathrm{H}$ & -5.877643000000 & 0.681866000000 & 0.189839000000 & $\mathrm{~s}$ & -3.403806000000 & -3.151923000000 & -0.017443000000 \\
\hline \multicolumn{4}{|c|}{$\begin{array}{c}\text { TS1 }_{\mathbf{A}}^{\prime \prime} \\
V_{\text {im. }}=-491.2 \mathrm{~cm}^{-1}(-1624,72805)\end{array}$} & \multicolumn{4}{|c|}{$\begin{array}{l}\text { TS2 } \\
0.8 \mathrm{~cm}^{-1}(-1624,73887)\end{array}$} \\
\hline C & 0.461732000000 & 0.314783000000 & 0.954657000000 & C & 0.837135000000 & 0.975127000000 & 0.566591000000 \\
\hline C & 0.217368000000 & -0.791397000000 & 0.071112000000 & C & 0.542179000000 & -0.098963000000 & -0.200430000000 \\
\hline C & -0.327877000000 & 1.416755000000 & 1.193773000000 & c & -0.106990000000 & 2.065872000000 & 0.974351000000 \\
\hline c & -0.975767000000 & -1.180886000000 & -0.608707000000 & c & -0.779621000000 & -0.443408000000 & -0.847675000000 \\
\hline $\mathrm{H}$ & -0.036215000000 & 2.143378000000 & 1.923488000000 & $\mathrm{H}$ & 0.412954000000 & 2.937197000000 & 1.338360000000 \\
\hline $\mathrm{H}$ & -1.369382000000 & 1.377153000000 & 0.946769000000 & $\mathrm{H}$ & -0.751748000000 & 1.715476000000 & 1.779015000000 \\
\hline 0 & -0.873780000000 & -1.670582000000 & -1.744752000000 & $\mathrm{o}$ & -0.631224000000 & -0.962852000000 & -2.051354000000 \\
\hline $\mathrm{N}$ & 1.776790000000 & 0.221133000000 & 1.368631000000 & $\mathrm{~N}$ & 2.157807000000 & 0.942695000000 & 0.955100000000 \\
\hline c & 1.506825000000 & -1.445004000000 & -0.145521000000 & C & 1.764844000000 & -0.860730000000 & -0.334167000000 \\
\hline c & 1.931000000000 & -2.520875000000 & -0.917048000000 & C & 2.103570000000 & -2.060838000000 & -0.973900000000 \\
\hline c & 2.434166000000 & -0.784932000000 & 0.681395000000 & c & 2.744102000000 & -0.163819000000 & 0.387736000000 \\
\hline
\end{tabular}


C $3.757615000000-1.166946000000$

C $3.269169000000-2.907652000000$

C $4.167116000000-2.239127000000$

H $\quad 1.233733000000-3.064590000000$

H 3.601815000000

H 5.197206000000

H 4.467023000000

C 0.225991000000

S -0.060170000000

Li 0.549073000000

H 1.761854000000

H 3.278803000000

H 2.802424000000

C -2.340589000000

C -2.563019000000

C -3.421127000000

C -3.849311000000

C -4.704320000000

C -4.918887000000

H -1.733764000000

H -3.246872000000

H -4.015803000000

H -5.534305000000

H -5.917669000000

S $\quad 0.594375000000$
$-3.738708000000$

$-2.546348000000$

$-0.657973000000$

2.851041000000

1.972956000000

$-0.506467000000$

1.179359000000

1.520771000000

0.701019000000

2.038257000000

$-1.036383000000$

$-1.177915000000$

$-0.838900000000$

$-1.111315000000$

$-0.752315000000$

$-0.890866000000$

$-1.348881000000$

$-0.754031000000$

$-1.231742000000$

$-0.585795000000$

$-0.832578000000$ 4.202772000000
C 2.441480000000

0.735988000000 $-0.865672000000$ $-0.054482000000$

$-1.527414000000$

$-1.461241000000$

$-0.029310000000$

1.361257000000

$-0.745240000000$

$-2.057034000000$

$-2.107867000000$

2.218636000000

2.986473000000

2.708206000000

1.659920000000

$-0.024495000000$

1.339494000000

$-0.877675000000$

1.846545000000

$-0.369336000000$

0.993733000000

1.999750000000

$-1.933522000000$

2.901864000000

$-1.032213000000$

1.388929000000

$-0.052635000000$

\section{5a}

$v_{\text {im. }}=0(-1624,71466)$

C $-0.633587000000-0.071913000000$

C $-0.194069000000 \quad 0.592376000000$

C $0.033801000000-1.204738000000$

C $1.064335000000 \quad 0.367654000000$

H $\quad-0.107239000000-1.144678000000$

H $1.095393000000-1.183117000000$

O $1.020884000000-0.079902000000$

$\begin{array}{lll}\text { N } & -1.896994000000 & 0.357178000000\end{array}$

C -1.256870000000

C -1.407852000000

C -2.290446000000

C -3.483271000000

C -2.583945000000

C -3.613580000000

H -0.625012000000

H -2.721044000000

H -4.524046000000

H -4.280563000000

C -0.299470000000

S -0.719694000000

Li 0.126424000000

C -2.776513000000

H -3.570024000000

H -2.223936000000

H -3.210695000000

C 2.378797000000

C 2.467575000000

C 3.546422000000

C 3.706647000000

C 4.779815000000

C 4.859217000000

H 1.573697000000

H 3.474036000000

H 3.769782000000

H $\quad 5.676736000000$

H $\quad 5.820987000000$

S -0.605150000000
1.470076000000

2.384347000000

1.297223000000

2.013060000000

3.090869000000

2.905129000000

2.526642000000

3.793233000000

3.468299000000

1.874712000000

$-2.739674000000$

$-3.949398000000$

$-1.789884000000$

$-0.165919000000$

$-0.760532000000$

$-0.788695000000$

0.653028000000

0.725669000000

1.535380000000

0.260868000000

1.874343000000

0.588706000000

1.397751000000

1.910034000000

$-0.356196000000$

2.507937000000

0.220136000000

1.656503000000

$-2.850347000000$
1.006326000000

$-0.098879000000$

1.720555000000

$-0.854709000000$

2.792238000000

1.530745000000

$-1.979583000000$

1.322851000000

$-0.523399000000$

$-1.569393000000$

0.399101000000

0.308277000000

$-1.665385000000$

$-0.731626000000$

$-2.292829000000$

$-2.467738000000$

$-0.832531000000$

$-0.483038000000$

$-1.545558000000$

2.347487000000

1.908411000000

3.032086000000

2.908919000000

$-0.254662000000$

0.872360000000

$-0.859576000000$

1.391576000000

$-0.334575000000$

0.791699000000

1.333502000000

$-1.734543000000$

2.257482000000

$-0.798154000000$

1.197809000000

1.201845000000
1.015104000000

$-2.182407000000$
C 4.062979000000

C 3.404404000000

C 4.376280000000

H 1.351558000000

H $\quad 3.679292000000$

H $\quad 5.385612000000$

H 4.819356000000

C -1.028077000000

S -1.650874000000

Li 0.552319000000

C 2.863448000000

H 2.227048000000

H 3.718411000000

H 3.206757000000

C -1.753043000000

C -1.639419000000

C -2.793054000000

C -2.555115000000

C -3.707073000000

C -3.594072000000

H -0.827129000000

H -2.868884000000

H -2.446567000000

H -4.506632000000

H -4.303667000000

S -1.428066000000
$-0.613822000000$

$-2.506562000000$

$-1.783566000000$

$-2.624402000000$

$-3.427465000000$

$-2.152980000000$

$-0.075490000000$

2.515155000000

1.318238000000

$-0.108792000000$

1.959769000000

2.367584000000

1.511871000000

2.768352000000

$-1.229497000000$

$-1.305522000000$

$-1.913280000000$

$-2.033164000000$

$-2.635000000000$

$-2.696075000000$

$-0.816590000000$

$-1.879113000000$

$-2.084938000000$

$-3.155254000000$

$-3.260983000000$

4.092825000000

$$
\text { Vim. }=-195.4(-1624,71198)
$$

C 0.840103000000

$-0.396980000000$

$-1.097075000000$

C $\quad 0.649004000000$

0.214792000000

C -0.193852000000

$-1.077218000000$

C -0.639572000000

0.392284000000

H $\quad 0.222260000000$

$-1.500102000000$

H -0.980806000000

O -0.635154000000

$-0.393955000000$

0.444417000000

N 2.176537000000

C 1.950977000000

C 2.405422000000

$-0.414410000000$

0.598544000000

1.261027000000

C 2.870676000000

C 4.239697000000

C 3.752882000000

0.192082000000

0.424112000000

1.490907000000

C 4.663167000000

H $\quad 1.707100000000$

H 4.118495000000

1.072317000000

1.578581000000

1.996930000000

1.260685000000

H 5.713013000000

H 4.952079000000

C -1.292259000000

0.109435000000

$-1.740523000000$

S -1.928854000000

$-2.658841000000$

Li -1.127163000000

C 2.772887000000

$-0.937382000000$

$-1.042912000000$

$-2.125463000000$

H 2.744642000000

H 2.268265000000

H 3.803372000000

$-0.733906000000$

$-0.732596000000$ 


\section{4a`}

$$
v_{\text {im. }}=0(-1624,77292)
$$

C $-1.021975000000-0.140733000000$

C $0.282819000000-0.102249000000$

C $-1.880893000000 \quad 0.992353000000$

C $1.046438000000 \quad 1.109050000000$

H $-2.101152000000 \quad 0.819259000000$

H $\quad-1.303010000000 \quad 1.907237000000$

$\begin{array}{lll}\text { O } & 0.532072000000 & 2.058260000000\end{array}$

N $\quad-1.417276000000-1.429107000000$

C $\quad 0.700235000000 \quad-1.477147000000$

C $1.851604000000-2.100662000000$

C $-0.400423000000-2.260810000000$

C $-0.390527000000-3.648683000000$

C $1.871918000000-3.472419000000$

C $\quad 0.758977000000-4.242145000000$

H $\quad 2.711981000000-1.527175000000$

H $2.753331000000-3.962579000000$

H $\quad 0.800267000000$

H -1.248624000000

C -3.202433000000

S -3.164922000000

Li -1.033740000000

C -2.706471000000

H -2.550320000000

H -3.297044000000

H -3.249444000000

C 2.476791000000

C 2.990120000000

C 3.299591000000

C 4.310292000000

C 4.622127000000

C 5.126202000000

H 2.359415000000

H 2.895518000000

H 4.698797000000

H $\quad 5.256524000000$

H $\quad 6.153763000000$

S -4.555127000000

1.465898000000
$-0.378924000000$ 0.073456000000 $-0.880989000000$ 0.360511000000 $-1.926234000000$ $-0.834587000000$ 0.934665000000 $-0.493783000000$ 0.272963000000 $-0.070715000000$ 0.041009000000 0.871094000000 0.510198000000 1.054396000000 1.243716000000 0.609864000000 $-0.216969000000$ $-0.141249000000$ 1.882072000000 $-0.949281000000$ $-1.642934000000$ $-0.108651000000$ $-1.452162000000$ $-0.034828000000$ $-1.105420000000$ 0.648420000000 $-1.487868000000$ 0.279778000000 $-0.791617000000$ $-1.645334000000$ 1.464199000000 $-2.323665000000$ 0.817114000000 $-1.084629000000$ $-1.048908000000$ 0.763783000000 1.579964000000

\section{$24 a$}

$V_{\text {im. }}=-0(-1624,76645)$

C $-2.653576000000-0.209021000000$

H -2.611620000000

C 2.061071000000

3.871345000000

\section{$V_{\text {im. }}=0(-1541,55242)$}

C 1.584511000000

C 0.566371000000

C 1.315707000000

C -0.775328000000

H 2.073288000000

N 2.835233000000

C 1.326688000000

C 0.958320000000

C 2.686831000000

C 3.678279000000

C 1.937989000000

C 3.282598000000

H -0.072771000000

H 1.660526000000

H 4.035444000000

H 4.720831000000

C -1.338054000000

S -2.963836000000

C 4.067594000000

H $\quad 4.077429000000$

H 4.899935000000

H 4.205707000000

C -1.742579000000

C -2.182262000000

C -2.193960000000

C -3.050397000000

C -3.061758000000
1.362758000000

0.315898000000

2.668274000000

0.550628000000

3.428310000000

0.769509000000

$-0.939939000000$

$-2.282826000000$

$-0.599234000000$

$-1.572247000000$

$-3.256110000000$

$-2.895718000000$

$-2.569192000000$

$-4.293689000000$

$-3.664061000000$

$-1.318772000000$

1.888075000000

2.224292000000

1.511482000000

2.283465000000

0.851246000000

1.975147000000

$-0.594305000000$

$-1.142032000000$

$-1.129735000000$

$-2.221236000000$

$-2.208988000000$
0.021761000000

0.013414000000

$-0.002967000000$

0.006621000000

$-0.011095000000$

0.051222000000

0.007773000000

$-0.009972000000$

0.016463000000

$-0.002861000000$

$-0.023953000000$

$-0.022139000000$

$-0.014801000000$

$-0.039001000000$

$-0.037117000000$

$-0.004954000000$

$-0.000857000000$

0.000585000000

$-0.009405000000$

0.752711000000

0.179655000000

$-0.983244000000$

0.004953000000

1.201130000000

$-1.192606000000$

1.201083000000

$-1.195202000000$
C $-0.493248000000 \quad 0.356913000000$

C $-0.571909000000-0.881142000000$

C $0.693447000000 \quad 1.257142000000$

C $0.485800000000 \quad-1.713166000000$

H $\quad 0.799899000000 \quad 1.531748000000$

H $\quad 1.596150000000 \quad 0.724640000000$

O $\quad 0.243889000000 \quad-2.381486000000$

N $\quad-1.739509000000 \quad 0.761135000000$

C $-1.970231000000-1.252466000000$

C $-2.679818000000-2.370526000000$

C $-4.033536000000-0.237760000000$

C $-4.041989000000-2.407987000000$

C $-4.713175000000-1.348567000000$

H $\quad-2.169926000000-3.173591000000$

H $\quad-4.604949000000-3.257755000000$

H $\quad-5.779424000000-1.402913000000$

H -4.556302000000

C 0.727799000000

S 0.082657000000

Li 1.026308000000

C -2.111555000000

H -2.775545000000

H -1.238596000000

C 1.862744000000

C 2.955461000000

C 3.337872000000

C 4.232222000000

C 4.424360000000

H 1.219411000000

H 2.793032000000

H 3.481946000000

H $\quad 5.074388000000$

H 5.416299000000

S 1.506801000000

0.572863000000

2.525465000000

2.515360000000

4.617617000000

2.032879000000

1.868280000000

2.661378000000

2.552568000000

$-1.780409000000$

$-1.795312000000$

$-1.915698000000$

$-1.936991000000$

$-2.031629000000$

$-2.045446000000$

$-1.711840000000$

$-1.929507000000$

$-1.962975000000$

$-2.120979000000$

$-2.145774000000$

$-0.440529000000$

0.131483000000

$-0.635080000000$

0.747886000000

$-1.677200000000$

$-0.367566000000$

1.708876000000

$-0.839049000000$

0.127158000000

0.573380000000

$-0.494103000000$

$-0.690272000000$

0.381706000000

$-0.244956000000$

1.069954000000

0.724245000000

$-0.375967000000$

$-1.163921000000$

0.209149000000

1.769256000000

1.652683000000

$-1.418787000000$

$-2.259001000000$

$-0.688816000000$

$-1.781824000000$

0.144617000000

$-1.229798000000$

0.994216000000

$-1.750435000000$

0.475104000000

$-0.898850000000$

$-1.893120000000$

2.055867000000

$-2.815759000000$

1.137790000000

$-1.302522000000$

$-0.476081000000$

$\mathbf{C S}_{2}$

$$
v_{\text {im. }}=0(-833,35437)
$$

C $0.000000000000 \quad 0.000000000000$

S $0.000000000000 \quad 0.000000000000$

0.000000000000

$-1.543253000000$

S 0.000000000000

1.543253000000 


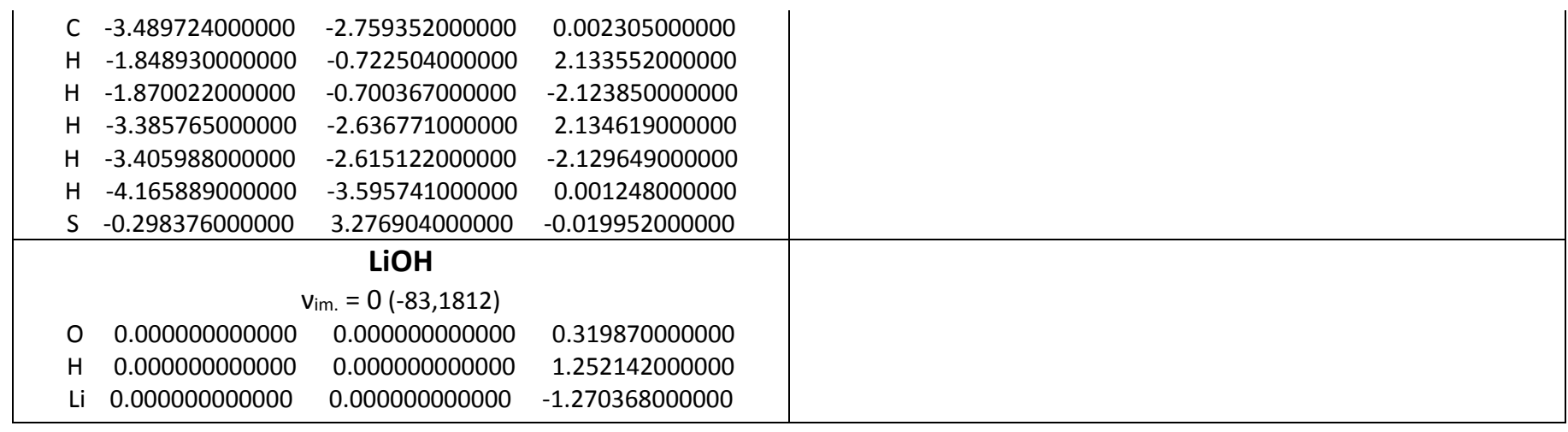

